\title{
Einstein equations and conformal structure: Existence of Anti-de Sitter-type space-times
}

\author{
Helmut Friedrich \\ Max-Planck Institut für Gravitations physik, Schlaatzweg 1, 14473 Potsdam, Germany
}

Received 23 August 1994

\begin{abstract}
We discuss Einstein's equations in the context of normal conformal Cartan connections, derive a new conformal representation of the equations, and express the equations in a conformally invariant gauge. The resulting formulation of the equations is used to show the existence of asymptotically simple solutions to Einstein's equations with a positive cosmological constant. The solutions are characterized by Cauchy data on a space-like slice and by the intrinsic conformal structure on the conformal boundary at space-like and null infinity.
\end{abstract}

Keywords: Einstein equations; Conformal structure

1991 MSC: 53A30; 83C05; 83C 15

\section{Introduction}

Einstein's field equations show a very peculiar transformation behaviour under conformal rescalings of the metric field. In a suitable representation the equations for the rescaled metric retain their hyperbolicity even under conformal rescalings with conformal factors which vanish on subsets of space-time. The consequences of this property for the longtime behaviour of gravitational fields have been worked out in detail for de Sitter-type space-times [5,7], i.e. for solutions to Einstein's field equations

$$
\operatorname{Ric}(\tilde{g})=\lambda \tilde{g}
$$

with negative cosmological constant $\lambda$ (the signature $(+,-,-,-)$ of the metric is assumed here, cf. Section 2 for our convention concerning the Ricci tensor). Beside the global nonlinear stability of the de Sitter space-time and other asymptotically simple solutions it has been shown that the conformal properties of the equations allow to deduce sharp and complete information on the fall-off behaviour of the gravitational field. 
The purpose of the present article is twofold. On the one hand our investigation of the "conformal structure of the field equations" will be completed in a certain sense. The artificially introduced gauge freedom of admitting arbitrary conformal rescalings will be extended by allowing in addition transitions to arbitrary symmetric connections which are compatible with the conformal structure of the metric $\tilde{g}$. From this results a new conformal representation of the Einstein equations (1.1). The purpose of this generalization is to increase the flexibility in choosing gauge conditions, in particular to admit a class of conformally invariant gauge conditions based on the use of conformal geodesics. These curves have been found useful before in the construction of coordinates in asymptotic regions of solutions to Einstein's equations [8]. The present article contains a systematic study of conformal geodesics in the context of the Einstein equations. It turns out that their use allows to specify explicitly certain quantities, which appear in the field equations and determine the gauge, in terms of the initial data. As a consequence the evolution equations get a surprisingly simple form which is ideally suited for a detailed analysis of the asymptotic behaviour of the solutions.

On the other hand we use the new conformal representation to analyse the behaviour of solutions to Einstein's field equations at large. The discussion of the conformal properties of Eqs. (1.1) in the first part of this article is sufficiently general to apply to cosmological constants of arbitrary sign. However, since the study of the case $\lambda=0$ requires a rather detailed analysis, it will be given in a separate article.

In the present paper we investigate solutions to (1.1) with $\lambda>0$. Although quite a number of properties of such solutions will be exhibited on the way, we shall focus on the question of existence of solutions, which apparently has not been addressed before in any generality. For investigations of such solutions concerned with their geometrical properties and directed towards applications to physics, the reader is referred to the article [12] and the references given therein.

We give an outline of our existence result. To describe the inital data, we consider sets $(\tilde{S}, \tilde{h}, \tilde{\chi})$ respectively $(S, h, \chi, \omega)$. Here $\tilde{S}, S$ denote smooth, orientable, three-dimensional manifolds, the latter being compact with boundary $\partial S$, while $\tilde{h}$ and $h$ denote smooth (negative definite) Riemannian metrics, $\tilde{\chi}$ and $\chi$ denote smooth, covariant, symmetric, rank 2 tensor fields on $\tilde{S}$ and $S$ respectively, and $\omega$ denotes a smooth function on $S$ which is positive on the interior and satisfies $\omega=0, \mathrm{~d} \omega \neq 0$ on $\partial S$.

A triple $(\tilde{S}, \tilde{h}, \tilde{\chi})$ is called a "smooth Cauchy data set for the Einstein equations", if the fields $\tilde{h}, \tilde{\chi}$ satisfy on $\tilde{S}$ the constraint equations induced by Eqs. (1.1) with some constant $\lambda>0$ on space-like hypersurfaces (cf. (5.1)). A smooth Cauchy data set $(\tilde{S}, \tilde{h}, \tilde{\chi})$ is called "smoothly conformally compactifiable with conformal extension $(S, h, \chi, \omega)$ ", if there exists an embedding $\tilde{S} \rightarrow S$ which allows one to identify $\tilde{S}$ with the interior $S \backslash \partial S$ of $S$ such that after this identification we have $h=\omega^{2} \tilde{h}, \chi=\omega \tilde{\chi}$ on $\tilde{S}$. In the theorem below we shall consider smoothly compactifiable Cauchy data sets which are "asymptotically simple", i.e. which satisfy the fall-off conditions inherent in the requirement of asymptotic simplicity, a notion which is discussed in detail in Section 5.1. The existence of a certain class of such data has been shown in [2]. 
With a smoothly conformally compactifiable Cauchy data set $(\tilde{S}, \tilde{h}, \tilde{\chi})$ with conformal extension $(S, h, \chi, \omega)$ we associate the manifold $M=S \times[0, \infty[$. We denote the part $\partial S \times[0, \infty[$ of its boundary by $\mathcal{I}$ and identify $S \equiv S \times\{0\}, \tilde{S} \equiv \tilde{S} \times\{0\}, \partial S \equiv \partial S \times\{0\}$. For given $\alpha \in \mathbb{R}^{+}$we set $\tilde{M}_{\alpha}=\tilde{S} \times\left[0, \alpha\left[\subset \tilde{M}, M_{\alpha}=S \times\left[0, \alpha\left[\supset \tilde{M}_{\alpha}, \mathcal{I}_{\alpha}=\mathcal{I} \cap M_{\alpha}\right.\right.\right.\right.$.

Up to some subtleties, which will be explained later on, we can now state the following theorem.

Theorem 1.1. Let $(\tilde{S}, \tilde{h}, \tilde{\chi})$ be a smoothly conformally compactifiable, asymptotically simple Cauchy data set for the Einstein equations (1.1) with positive cosmological constant $\lambda$ which has conformal extension $(S, h, \chi, \omega)$. On $\mathcal{I}$ let there be given a smooth Lorentzian conformal structure $\mathcal{C}_{\mathcal{I}}$ of signature $(+,-,-)$. Suppose the data $\tilde{h}, \tilde{\chi}, \mathcal{C}_{\mathcal{I}}$ satisfy on $\partial S$ the corner conditions (cf. Section 5.4). Then there exists for some $\alpha>0$ a solution $\tilde{g}$ to the Einstein equations (1.1) on $\tilde{M}_{\alpha}$ with the following properties. The metric $\tilde{g}$ induces $\tilde{h}$ as the first and $\tilde{\chi}$ as the second fundamental form on $\tilde{S}$ and for any smooth function $\Omega$ on $M_{\alpha}$ with $\Omega>0$ on $\tilde{M}_{\alpha}, \Omega=0, \mathrm{~d} \Omega \neq 0$ on $\mathcal{I}_{\alpha}$, the metric $\Omega^{2} \tilde{g}$ on $\tilde{M}_{\alpha}$ extends to a smooth Lorentz metric $g$ on $M_{\alpha}$ which induces on $\mathcal{I}_{\alpha}$ a conformal structure which coincides with the restriction of $\mathcal{C}_{\mathcal{I}}$ to $\mathcal{I}_{\alpha}$. The solution $\tilde{g}$ is unique up to diffeomorphisms and extensions.

The theorem asserts the existence of solutions to quite an unusual type of initial boundary value problems for Einstein's equations. Null geodesics with respect to the metric $\tilde{g}$ approach end points on $\mathcal{I}_{\alpha}$ only after infinite "time", as measured in terms of an affine parameter. Therefore any such null geodesic is future complete. The example of Anti-de Sitter space-time (cf. Section 4) suggests that time-like geodesics never end on $\mathcal{I}_{\alpha}$, while space-like geodesics may do so. Again, in the direction in which the latter approach $\mathcal{I}_{\alpha}$, they are complete. Thus $\mathcal{I}_{\alpha}$ represents a boundary at space-like and null infinity. The boundary data, which are provided by the restriction of the conformal structure $\mathcal{C}_{\mathcal{I}}$ to the set $\mathcal{I}_{\alpha}$, are thus prescribed on a cylinder which represents space-like and null infinity for the solution $\left(\tilde{M}_{\alpha}, \tilde{g}\right)$. The solutions considered here will be called "Anti-de Sitter-type (AdS-type) solutions".

The "location of infinity" is determined solely in terms of the solution $\tilde{g}$ to the quasi-linear field equations whose existence we want to establish. Thus we are dealing with a kind of free boundary value problem and much of our discussion is devoted to the reduction of this problem to an initial boundary value problem with a fixed boundary. Another major part of the analysis is concerned with the question which data may be prescribed on the boundary at infinity, a problem which has not been investigated so far.

Neither the equations nor our initial and boundary conditions prefer a time direction. Therefore our result implies for suitably extended boundary data also the existence of an extension of the solution into the past of the initial hypersurface $\tilde{S}$. The solutions so obtained could be called global in space-like directions. Contrary to the case of solutions to Einstein's equations with vanishing cosmological constant, in the present case this notion presumes the existence of null geodesics which are complete in the future or in the past and whose end points generate the boundary $\mathcal{I}$. 
The existence of solutions of this type has first been conjectured by Penrose [15,16]. They represent space-times which he called "asymptotically simple". The hypersurface $\mathcal{I}$ denotes their conformal boundary. Up to questions of extensibility, all such solutions which are smooth have been characterized here by initial data on a space-like hypersurface and boundary data on $\mathcal{I}$. As has been recognized before in the case of a negative cosmological constant [5,7], it is seen now that the concept of asymptotic simplicity is natural for Einstein's field equations also in the case of a positive cosmological constant.

In a certain context Hawking [10] suggested as a boundary condition for solutions to Einstein's equations with positive cosmological constant that the intrinsic conformal structure on the conformal boundary $\mathcal{I}$ be conformally flat . This condition is a special case of our boundary conditions. Note that it slightly restricts the Cauchy data on the spacelike slice by the corner conditions. However, such conditions are not peculiar to the special situation considered here, they occur naturally in any initial boundary value problem.

It appears difficult to give a simple description and treatment of the initial boundary value problem considered here without referring, explicitly or implicitly, to the conformal structure. Null geodesics, which appear to suggest themselves for this purpose, are not particularly helpful. In regions sufficiently far into the future of the initial hypersurface, null geodesics may be expected to have past as well as future end points on $\mathcal{I}$ in the future of $\tilde{S}$ and therefore can hardly be used to control the behaviour of the solution in terms of the initial and boundary data. To illustrate the point, we indicate in Section 7.2 how the boundary data are described by using conformal geodesics, which satisfy a system of equations given in terms of the metric $\tilde{g}$.

Nevertheless, at the expense of an enormous technical complication it may be possible to rederive the above result in terms of the "physical" metric $\tilde{g}$ alone. This raises the question, whether anything may be gained by dispensing with the conformal techniques. It may be asked in particular, whether the condition of asymptotic simplicity, which may appear an excessively strong requirement on the data, is imposed only to allow the application of the conformal methods. As discussed at the end of Section 5.1, there can be constructed smoothly conformally compactifiable initial data sets for Eqs. (1.1) which do not satisfy the condition of asymptotic simplicity [2]. In that case the conformal curvature diverges on open subsets of the boundary $\partial S$. Most likely this behaviour spreads into space-time, thus restricting severely the extensibility of the solution beyond the domain of dependence of the initial hypersurface $\tilde{S}$.

In this context it is worthwhile to remark also on the issue of the global existence of solutions to the Einstein equations with a positive cosmological constant. This problem is of quite a different nature as the corresponding problems where the cosmological constant is non-positive, whereas in those cases the global solutions are "conformally finite", this is not true any longer in the case of a positive cosmological constant (cf. Section 4).

The natural starting point for our discussion of the conformal representation of the Einstein equations is provided by Cartan's theory of conformal connections, which is reviewed in some detail in Section 2. In Section 3 it is used to obtain the desired conformal representation of the Einstein equations and the essential properties of conformal geodesics. The results on conformal geodesics which are decisive for their use in the conformal Einstein 
equations are derived in Lemmas 3.1 and 3.2.

In Section 6 it is shown that solutions to the reduced conformal field equations indeed provide solutions to Einstein's equations if appropriate data are prescribed. This discussion allows to trace the relationship of the present conformal representation of the Einstein equations with their conformal representation used in [5,7]. We shall, however, not comment on it.

Section 5 contains the critical step in our treatment of the problem. Using the previous results we show that the semiglobal problem considered in the theorem can be reduced to the analysis of local initial boundary value problems for the conformal field equations. Another application of Cartan's theory, this time to a three-dimensional problem, is made in Section 7, where it is shown how the covariant boundary conditions considered in Theorem 1.1 are related to the "maximal dissipative" boundary conditions which are considered in Section 5.

The initial boundary value problem for a symmetric hyperbolic system obtained in Section 5 is quite close to, though not quite identical with, a type of mixed problem studied by Guès [9]. Nevertheless, as discussed in Section 8, Guès' results carry over to the present case and imply the existence of solutions to the general mixed problem formulated in Section 5 and consequently of the problem considered in Theorem 1.1 .

It appears, that this article contains the first general treatment of an initial boundary value problem for Einstein's field equations. To some extent our analysis relies on special features of the conformal boundary. It may be useful, though, to point out the general properties of the field equations observed in Sections 5-7 which determine the nature of the boundary conditions.

\section{The normal conformal Cartan connection}

In the following we consider a smooth $n$-dimensional manifold $M, n \geq 3$, endowed with a smooth conformal structure $\mathcal{C}$. We think of $\mathcal{C}$ as being given by a class of smooth metrics of signature $(p, q)$ on $M, p+q=n$, such that two metrics $g, \tilde{g} \in \mathcal{C}$ are related by a smooth conformal rescaling

$$
\tilde{g}=\Omega^{2} g, \quad \Omega \in C^{\infty}(M), \quad \Omega>0
$$

The pair $(M, \mathcal{C})$ will be called a conformal space.

With the conformal structure $\mathcal{C}$ there is associated the "normal conformal Cartan connection" on a bundle $H(M)$ of second-order frames over $M$. In this section we shall outline its construction and those of its properties which will be needed later on. For further details, and for some discussion of the case of a Lorentzian conformal structure $(p=1, n=4)$ we refer the reader to $[8,13,14]$ and the literature cited therein. Our representation of the subject will be geared to later applications, therefore a number of transformation formulas, etc. are explicitly given. 


\subsection{Some conformal groups, Lie algebras, etc.}

We call the pseudo Riemannian space with manifold $M_{*}=\mathbb{R}^{n}$ and metric $g_{*}=\eta_{i j} \mathrm{~d} x^{i} \mathrm{~d} x^{j}$, where the $x^{i}$ are the standard coordinates on $\mathbb{R}^{n}$ and $\eta_{i j}=0$ if $i \neq j, \eta_{i j}=1$ if $i=j=$ $1, \ldots, p, \eta_{i j}=-1$ if $i=j=p+1, \ldots, n$, the "flat standard structure of signature $(p, q)$ ". The point of $M_{*}$ where $x^{i}=0$ will be called the "origin" of this space and denoted by $o$. The (local) group $H(p, q)$ of transformations $f$ which smoothly map a neighbourhood $U$ of the origin onto another such neighbourhood $U^{\prime}$, such that the origin is left fixed and the conformal structure induced by $g_{*}$ on $U, U^{\prime}$ respectively is preserved, i.e.

$$
{ }^{\mathrm{t}} f g_{*}=\Omega^{2} g_{*}, \quad \Omega \in C^{\infty}(U), \Omega>0
$$

is generated by:

(i) The group $C O(p, q)$ of smooth conformal maps of $\left(M_{*}, g_{*}\right)$ into itself. An element of this group is given by a matrix $C$ of the form $C=\Omega^{-1} A$, where $\Omega \in \mathbb{R}^{+}$and $A$ satisfies

$$
\eta_{i j} A_{k}^{i} A^{j}{ }_{l}=\eta_{k l},
$$

i.e. $A$ is in the subgroup $O(p, q)$ of isometries of the flat standard structure which leave the origin fixed.

(ii) The (local) group of "special conformal transformations". An element of this group is characterized by some $a \in \mathbb{R}^{n}$ and acts near the origin by

$$
x^{i} \longrightarrow \frac{x^{i}+\frac{1}{2} a^{i} \eta(x, x)}{1+\eta(x, a)+\frac{1}{4} \eta(x, x) \eta(a, a)},
$$

where $\eta(x, a) \equiv \eta_{i k} x^{i} a^{k}$, etc. Since these transformations are not defined everywhere on $M_{*}, H(p, q)$ does not act as a group of conformal transformations on the flat standard structure. In the following, two methods will be studied by which $H(p, q)$ is made into a transformation group.

The first way of doing this is useful for the discussion of group theoretical properties. In this case the flat standard structure is conformally embedded into a conformal space, the Möbius space, which by its construction allows to extend the local action of $H(p, q)$ to the whole space. Consider the space with manifold $\mathbb{R}^{n+2}$ and metric $\bar{g}=B_{A C} \mathrm{~d} x^{A} \mathrm{~d} x^{C}$, where $x^{A}, A=0,1, \ldots, n+1$ are the standard coordinates on $\mathbb{R}^{n+2}$ and the coefficients of the metric are given by the matrix

$$
B=\left(B_{A C}\right)=\left(\begin{array}{ccc}
0 & 0 & -1 \\
0 & \eta_{i j} & 0 \\
-1 & 0 & 0
\end{array}\right)
$$

The metric induced by $\bar{g}$ on the set $N_{p, q}=\left\{x \in \mathbb{R}^{n+2} \backslash\{0\} \mid B_{A C} x^{A} x^{C}=0\right\}$ is degenerate in the direction of the "null generators"

$$
\lambda \longrightarrow \lambda x, \quad \lambda \in \mathbb{R}^{*}, \quad x \in N_{p, q} .
$$


Using the action of $\mathbb{R}^{*}$ on $N_{p, q}$ indicated by (2.1), we consider the quotient space $Q_{p, q}=$ $N_{p, q} / \mathbb{R}^{*}$. It may be identified with the quadric in $P^{n+1}(\mathbb{R})$ defined by the quadratic form associated with $B$. From $\left(\mathbb{R}^{n+2}, \bar{g}\right)$ it inherits a conformal structure into which the flat standard structure of signature $(p, q)$ can be conformally embedded. In homogeneous coordinates, in which $Q_{p, q}$ is represented by $N_{p, q}$, such an embedding is realized by the map

$$
\mathbb{R}^{n} \ni x^{i} \stackrel{F}{\longrightarrow} \Omega(x)\left(\frac{1}{2} \eta(x, x), x^{i}, 1\right) \in N_{p, q},
$$

which satisfies ${ }^{t} F \bar{g}=\Omega^{2} g_{*}$. Here $\Omega \in C^{\infty}\left(M_{*}\right)$ is an arbitrary positive function. The manifold $Q_{p, q}$ endowed with this conformal structure is the "Möbius space of signature $(p, q)$ ". The group $O(p+1, q+1)$ of isometries of $\bar{g}$ which leave the origin fixed is given by matrices $A$ satisfying ${ }^{\mathrm{t}} A B A=B$. Since these maps also leave fixed the set $N_{p, q}$, they induce conformal maps of $Q_{p, q}$ into itself. The mappings so obtained form the "Möbius group" $C(p, q) \cong O(p+1, q+1) /\{E,-E\}$, which comprises in fact all conformal maps of $Q_{p, q}$. Here, as for other matrix groups considered in this article, $E$ denotes the unit element. The embedding (2.2) with $\Omega=1$ shows that the subgroup $K(p+1, q+1)$ of $O(p+1, q+1)$ which leaves fixed the ray $\mathbb{R} \cdot(0, \ldots, 0,1)$ in $\mathbb{R}^{n+2}$ induces the local transformations of $H(p, q)$ on the embedded flat standard structure. The transformations in $H(p, q)$ thus extend to all of $Q_{p, q}$. It is furthermore seen that the group $H(p, q)$ is the semi-direct product $C O(p, q) \times_{\sigma} \mathbb{R}^{n *}$ with multiplication defined by

$$
(C, b)\left(C^{\prime}, b^{\prime}\right)=\left(C C^{\prime}, b C^{\prime}+b^{\prime}\right), \quad C, C^{\prime} \in C O(p, q), \quad b, b^{\prime} \in \mathbb{R}^{n *}
$$

and we have a $2: 1$ group homomorphism

$$
K(p+1, q+1) \ni \pm\left(\begin{array}{ccc}
\Omega^{-1} & 0 & 0 \\
\Omega^{-1} f^{i} & A_{k}^{i} & 0 \\
\Omega^{-1} \frac{1}{2} f^{l} f_{l} & f_{l} A_{k}^{l} & \Omega
\end{array}\right) \longrightarrow(C, f C) \in H(p, q),
$$

where $C=\Omega^{-1} A, \Omega \in \mathbb{R}^{+}, A \in O(p, q), f \in \mathbb{R}^{n *}$, and indices are moved, as will be done in the following, with $\eta_{i j}$.

We shall use the decomposition of the Lie algebra $o(p+1, q+1) \cong c(p, q)$ (cf. [13] for more details) described in the matrix notation above by the $1: 1$ map

$$
\begin{aligned}
& o(p+1, q+1) \ni\left(\begin{array}{ccc}
-\omega & z_{j} & 0 \\
d^{i} & u^{i}{ }_{j} & z^{i} \\
0 & d_{j} & \omega
\end{array}\right) \\
& \rightarrow\left(z^{i}, u^{i}{ }_{j}-\omega \delta^{i}{ }_{j}, b_{j}\right) \in \mathbb{R}^{n} \oplus c o(p, q) \oplus \mathbb{R}^{n *} \cong c(p, q),
\end{aligned}
$$

where $u^{i}{ }_{j} \in o(p, q), \omega \in \mathbb{R}$.

To describe the adjoint action $A d$ of the group $H(p, q)$ on $c(p, q)$ we need the map

$$
\mathbb{R}^{n *} \ni b \longrightarrow S(b) \in \mathbb{R}^{n *} \otimes \mathbb{R}^{n} \otimes \mathbb{R}^{n *}
$$

which is defined, in index notation, by

$$
b_{j} \longrightarrow S(b)_{i}{ }^{k}{ }_{l} \equiv \delta^{k}{ }_{i} b_{l}+\delta^{k}{ }_{l} b_{i}-\eta_{i l} \eta^{k j} b_{j} .
$$


It is important that the expression on the right is symmetric in the lower indices and takes, for fixed index $i$, values in $c o(p, q)$. If $f \in \mathbb{R}^{n *}, z \in \mathbb{R}^{n}$, we denote the contraction of $f$ with $S(b)$ by $\langle f, S(b)\rangle$, and the contraction of $z$ with $S(b)$ by $S(b) z$, such that we have, e.g. $\langle f, S(b) z\rangle_{i}=f_{k}\left(\delta^{k}{ }_{i} b_{l}+\delta^{k}{ }_{l} b_{i}-\eta_{i l} \eta^{k j} b_{j}\right) z^{l}$. An important identity needed in various calculations is given by

$$
\operatorname{Ad}\left(C^{-1}\right)(S(b) C z)=S(b C) z, \quad z \in \mathbb{R}^{n}, \quad C \in C O(p, q), \quad b \in \mathbb{R}^{n *} .
$$

Observing that the adjoint action of $K(p+1, q+1)$ on $c(p, q)$ factorizes as

$$
K(p+1, q+1) \stackrel{2: 1}{\longrightarrow} H(p, q) \stackrel{A d}{\longrightarrow} G L\left(\mathbb{R}^{n} \oplus \operatorname{co}(p, q) \oplus \mathbb{R}^{n *}\right),
$$

we can deduce the map $A d$ from the matrix representation above. For $s=(C, f C) \epsilon$ $H(p, q)$ and $z \in \mathbb{R}^{n}, U \in o(p, q), \omega \in \mathbb{R}, d \in \mathbb{R}^{n *}$, we get

$$
\begin{aligned}
A d\left(s^{-1}\right)(z, U-\omega E, d)= & \left(C^{-1} z, C^{-1}(S(f) z+U-\omega E) C,\right. \\
& \left.\left(-\frac{1}{2}\langle f, S(f) z\rangle-f(U-\omega E)+d\right) C\right) .
\end{aligned}
$$

From this ensues, for $z \in \mathbb{R}^{n}, \quad U, V \in \operatorname{co}(p, q), \omega, \alpha \in \mathbb{R}, d, b \in \mathbb{R}^{n *}$, the commutator

$$
\begin{aligned}
& {[(0, V-\alpha E, b),(z, U-\omega E, d)]} \\
& \quad=((V-\alpha E) z,-S(b) z+[V, U], b(U-\omega E)-d(V-\alpha E))
\end{aligned}
$$

in the Lie algebra $c(p, q)$. The maps $A d(s), s \in H(p, q)$, respect the decomposition of $c(p, q)$ given above in the sense that

$$
\operatorname{Ad}(s)(\operatorname{co}(p, q)) \subset \operatorname{co}(p, q) \oplus \mathbb{R}^{n *}, \quad \operatorname{Ad}(s)\left(\mathbb{R}^{n *}\right) \subset \mathbb{R}^{n *} .
$$

Furthermore, it follows from the formulae above that $A d\left(s^{-1}\right), s \in H(p, q)$, induces a map of $\mathbb{R}^{n} \oplus \operatorname{co}(p, q) \oplus\{0\} \cong o(p+1, q+1) / \mathbb{R}^{n *}$ into itself, which we denote by $\overline{A d}\left(s^{-1}\right)$, and which is given for $s=(C, f C)$ by

$$
(z, U-\omega E, 0) \rightarrow\left(C^{-1} z, C^{-1}(S(f) z+U-\omega E) C, 0\right) .
$$

We denote by $\omega=\left(\sigma^{i}, \omega^{i}{ }_{j}, \omega_{j}\right)$ the $\left(\mathbb{R}^{n} \oplus \operatorname{co}(p, q) \oplus \mathbb{R}^{n *}\right)$-valued Maurer-Cartan form on the Möbius group $C(p, q)$. The explicit form of the Maurer-Cartan equation

$$
\mathrm{d} \omega=-[\omega, \omega]
$$

can be determined from the commutator (2.6) as

$$
\begin{aligned}
& \mathrm{d} \sigma^{i}=-\omega^{i}{ }_{k} \wedge \sigma^{k}, \\
& \mathrm{~d} \omega_{k}^{i}=-\omega^{i}{ }_{l} \wedge \omega_{k}{ }_{k}+S\left(\omega_{p}\right)_{q}{ }_{k}{ }_{k} \wedge \sigma^{q}, \\
& \mathrm{~d} \omega_{k}=-\omega_{i} \wedge \omega^{i}{ }_{k},
\end{aligned}
$$

where we use an obvious extension of the map (2.3) to a map from $\mathbb{R}^{n *}$-valued $r$-forms to $\mathbb{R}^{n *} \otimes \mathbb{R}^{n} \otimes \mathbb{R}^{n *}$-valued $r$-forms, $r=0,1,2, \ldots$ 


\subsection{The bundle $H(M)$ of second-order frames}

The second way to make $H(p, q)$ into a transformation group is suggested by the following observation. Since $f \in H(p, q)$ leaves the origin fixed, its tangent map $T_{o}(f)$ at that point acts as a linear transformation on the tangent space $T_{o}\left(M_{*}\right)$. The elements in $C O(p, q)$ are completely characterized by this action. To characterize in a similar way the special conformal transformations $f$, which induce the identity transformation on $T_{o}\left(M_{*}\right)$, we investigate their effect on a structure of higher order. Such a structure is given by those subspaces of the tangent spaces $T_{h}\left(T M_{*}\right)$ at points $h \in T_{o}\left(M_{*}\right)$ which are horizontal with respect to the Levi-Civita connections associated with metrics conformal to $g_{*}$. These subspaces are mapped into each other by the tangent maps $T_{o}(f), f \in H(p, q)$, and the group of special conformal transformations acts effectively and transitively on them. This action, which generalizes to general conformal structures, will be studied in the following.

Given the conformal structure $\mathcal{C}$ on $M$ we denote by $C O(M)$ the principal bundle of conformal frames $f_{x}=\left(x,\left\{e_{k}\right\}_{k=0,1, \ldots, q=n-1}\right)$ with $x \in M, e_{k} \in T_{x} M$, such that for $g \in \mathcal{C}$ there is an $a \in \mathbb{R}^{+}$with

$$
g\left(e_{i}, e_{j}\right)=a^{2} \eta_{i j}
$$

Its structure group is $C O(p, q)$ and its projection to $M$ will be denoted by $\pi^{\prime}$. We shall consider a frame alternatively as an $n$-tupel of tangent vectors or as the isomorphism given by $\mathbb{R}^{n} \ni z \rightarrow z^{i} e_{i} \in T_{x}(M)$. We also identify a frame field with the local section it defines.

Let $\left\{x^{\mu}\right\}_{\mu=1,2, \ldots, n}$ be a local coordinate system on an open subset $U$ of $M$ which maps $U$ onto an open set $V \subset \mathbb{R}^{n}, e_{i}=e^{\mu}{ }_{i}(x) \partial_{\mu}, i=1,2, \ldots, n$, a frame field defining a local section of $C O(M)$ over $U$, and $\sigma^{j}$ the dual 1-form field satisfying

$$
\left\langle\sigma^{i}, e_{j}\right\rangle=\left\langle\sigma^{i}{ }_{\nu} \mathrm{d} x^{\nu}, e^{\mu}{ }_{j} \partial_{\mu}\right\rangle=\sigma_{\mu}^{i} e^{\mu}{ }_{j}=\delta^{i}{ }_{j}
$$

A local bundle coordinate system is given by

$$
C O(M) \supset \pi^{\prime-1}(U) \ni e_{i}(x) C^{i}{ }_{j} \longrightarrow\left(x^{\mu}, C^{i}{ }_{j}\right) \in V \times C O(p, q) .
$$

We denote the Killing vector field generated by $u \in \operatorname{co}(p, q)$ on $C O(M)$ by $Z_{u}$ and the $\mathbb{R}^{n}$-valued solder form on $C O(M)$ by $\bar{\sigma}^{j}$.

Suppose $\nabla$ is a connection on $M$ which is torsion free, i.e. it holds

$$
\nabla_{e_{i}} e_{l}-\nabla_{e_{l}} e_{i}=\left[e_{i}, e_{l}\right]
$$

and which respects the conformal class in the sense that for any $g \in \mathcal{C}$ we have

$$
\nabla_{\lambda} g_{\mu \nu}=-2 d_{\lambda} g_{\mu \nu},
$$

where $d_{\lambda} \mathrm{d} x^{\lambda}$ is a 1 -form on $M$ which depends on $g$. Such a connection is sometimes called a "Weyl connection". However, in order to avoid any (misleading) allusion to Weyl's theory of gravitation [21] we will call such a connection "conformal for $g$ (for $\mathcal{C}$ )". It is not required to be metric with respect to any metric in the conformal class $\mathcal{C}$. A connection with these 
properties can be represented by a torsion free $c o(p, q)$-valued connection form $\omega^{i}{ }_{j}$ on $C O(M)$ and $\nabla$ will also be called "torsion free connection on $C O(M)$ ".

For given $z \in \mathbb{R}^{n}$ the connection $\nabla$ distinguishes on $C O(M)$ a "horizontal vector field" $H_{z}$ by the requirements $\left\langle\omega, H_{z}\right\rangle=0,\left\langle\bar{\sigma}^{j}, H_{z}\right\rangle=z^{j}$. Defining for given $f_{x} \in C O(M)$ an isomorphism, whence a frame on $C O(M)$, by

$$
F_{\nabla}\left(f_{x}\right):\left\{\begin{array}{l}
\mathbb{R}^{n} \oplus \operatorname{co}(p, q) \longrightarrow T_{f_{x}}(C O(M)), \\
(z, u) \longrightarrow H_{z}\left(f_{x}\right)+Z_{u}\left(f_{x}\right)
\end{array}\right.
$$

we obtain a section $C O(M) \ni f_{x} \rightarrow F_{\nabla}\left(f_{x}\right) \in F C O(M)$ of the bundle $F C O(M)$ of frames of $C O(M)$.

The connection coefficients of $\nabla$ with respect to the frame field $e_{k}$ are defined on $U$ by $\Gamma_{i}{ }^{k}{ }_{l} e_{k}=\nabla_{e_{i}} e_{l}$. Suppose that $\nabla^{*}$ is another torsion free connection on $C O(M)$ and let $\Gamma_{i}^{* k}{ }_{l}$ denote its connection coefficients with respect to $e_{k}$. From the fact that the connections are torsion free ensues, that the components of the difference tensor $\nabla^{*}-\nabla$ with respect to the frame field $e_{k}$ are given on $U$ by $\Gamma_{i}^{* k}{ }_{l}-\Gamma_{i}{ }^{k} l=S(b)_{i}{ }^{k}{ }_{l}$. Here $b_{k}$ is some $\mathbb{R}^{n \cdot *}$-valued function on $U$ which is given by the components of a 1 -form $b$ on $M$ with respect to the frame $e_{k}$, i.e. $b_{k}=\left\langle b, e_{k}\right\rangle=b_{\mu} e^{\mu}$. The 1 -form $b$ is defined uniquely and everywhere on $M$ by the connections $\nabla, \nabla^{*}$.

Suppose, we are given connections $\nabla, \nabla^{*}$ as above and conformal frame fields $f=$ $\left\{e_{k}\right\}_{k=1, \ldots, n}$ and $\bar{f}=\left\{\bar{e}_{k}\right\}_{k=1, \ldots, n}$ on $U$. Denote the connection coefficients of $\nabla$ with respect to $f$ by $\Gamma$ and with respect to $\bar{f}$ by $\bar{\Gamma}$ and those of $\nabla^{*}$ with respect to $f$ by $\Gamma^{*}$ and with respect to $\bar{f}$ by $\bar{\Gamma}^{*}$. If $\bar{e}_{k}=e_{j} C^{j}{ }_{k}$ with some $C O(p, q)$-valued function $C^{i}{ }_{j}$ on $U$, then we have the well-known formula $\bar{\Gamma}_{i}{ }_{k}=\left(C^{-1}\right)^{j}{ }_{l}\left(\bar{e}_{i}\left(C^{l}{ }_{k}\right)+\Gamma_{p}{ }^{l}{ }_{q} C^{p}{ }_{i} C^{q}{ }_{k}\right)$ and a similar formula for the starred quantities. It follows $S(\bar{b})_{i}{ }^{j}{ }_{k} \equiv \bar{\Gamma}_{i}^{* j}{ }_{k}-\bar{\Gamma}_{i}{ }^{j}{ }_{k}=S(b C)_{i}{ }_{k}{ }_{k}$ which corresponds to the transformation behaviour of $b_{k}$ under transformations of the frame.

If we denote by $H_{z}^{*}$ the vector fields horizontal for $\nabla^{*}$, we get the equation

$$
H_{z}^{*}\left(f_{x}\right)=H_{z}\left(f_{x}\right)-Z_{S\left(b\left(f_{x}\right) z\right)}\left(f_{x}\right), \quad z \in \mathbb{R}^{n}
$$

where $b$ denotes the 1 -form on $M$ defining the difference tensor of $\nabla, \nabla^{*}$ and $b\left(f_{x}\right)$ the $\mathbb{R}^{n *}$-valued function such that ${ }^{\mathrm{t}} \pi^{\prime} b=b_{j}\left(f_{x}\right) \bar{\sigma}^{j}$. Equivalently, if $\omega^{i}{ }_{j}, \omega^{* i}{ }_{j}$ denote the connection forms defined on $C O(M)$ by the connections $\nabla, \nabla^{*}$ respectively, then $\omega^{* i}{ }_{j}=$ $\omega^{i}{ }_{j}+S\left(b\left(f_{x}\right)\right)_{k}{ }_{j}{ }_{j} \bar{\sigma}^{k}$. For $b \in \mathbb{R}^{n *}$ we consider at $f_{x} \in C O(M)$ the frame

$$
F_{f_{x}, b}:(z, u) \longrightarrow H_{z}\left(f_{x}\right)+Z_{u}\left(f_{x}\right)+Z_{S(b) z}\left(f_{x}\right)
$$

The set $H(M)$ of these frames defines a subbundle of the bundle of frames of $C O(M)$ with projection $q: F_{f_{x}, b} \rightarrow f_{x}$. We identify $\mathbb{R}^{n *}$ with a subgroup of $G L\left(\mathbb{R}^{n} \oplus \operatorname{co}(p, q)\right)$ by

$$
\mathbb{R}^{n *} \ni b \longrightarrow\left(\begin{array}{cc}
i d_{\mathbb{R}^{n *}} & 0 \\
-S(b) & i d_{c o(p, q)}
\end{array}\right) \ni G L\left(\mathbb{R}^{n} \oplus \operatorname{co}(p, q)\right) .
$$

The resulting action of $\mathbb{R}^{n *}$ on the bundle of frames of $C O(M)$ gives for $b \in \mathbb{R}^{n *}$ 


$$
\begin{aligned}
\left(\left(F\left(f_{x}\right)\right) b\right)(z, u) & =F\left(f_{x}\right)(z,-S(b) z+u) \\
& =H_{z}\left(f_{x}\right)+Z_{u}\left(f_{x}\right)-Z_{S(b) z}\left(f_{x}\right) .
\end{aligned}
$$

With the action so described the bundle $(H(M), C O(M), q)$ acquires the structure of a principal fibre bundle with structure group $\mathbb{R}^{n *}$. The frame $F_{\nabla}$ defined on $C O(M)$ by the connection $\nabla$, the frame $\left\{e_{k}\right\}_{k=0, \ldots, 4}$ with respect to which the connection coefficients have been given, and the coordinates $x^{\mu}$ define a bundle coordinate system

$$
\begin{aligned}
H_{0}(M) \supset q^{-1}\left(\pi^{\prime-1}(U)\right) \ni\left(F_{\nabla}\left(f_{x}\right)\right) b & \longrightarrow\left(f_{x}, b\right) \\
& \longrightarrow(x, C, b) \in V \times C O(p, q) \times \mathbb{R}^{n *} .
\end{aligned}
$$

The transformation law for the horizontal vector fields under the transition $\nabla \rightarrow \nabla^{*}$ shows that the definition of the bundle $(H(M), C O(M), q)$ is independent of the connection which has been chosen to describe the construction of the bundle. From the same relation follows also, that each torsion free connection on $C O(M)$ defines a section of $H(M)$ and vice versa.

We shall discuss now a smooth action of the group $H(p, q)$ on $H(M)$ by which the latter becomes the space of a principal fibre bundle over $M$ with structure group $H(p, q)$ and projection $\pi: F\left(f_{x}\right) \rightarrow x$. Any $C \in C O(p, q)$ acts on $C O(M)$ by the map $R_{C}: f_{x} \rightarrow$ $f_{x} C \equiv\left(x, e_{k} C_{j}^{k}\right)$. We denote its tangent map by $T\left(R_{C}\right)$. Using the action $(C,(z, u)) \rightarrow$ $\overline{A d}(C)(z, u)=(C z, A d(C) u)$ of $C O(p, q)$ on $\mathbb{R}^{n} \oplus c o(p, q)$, we can define a right action of $C O(p, q)$ on $H(M)$ by $(F C)(z, u)=T\left(R_{C}\right)\{F(\overline{A d}(C)(z, u))\}$. The action on the right of the group $H(p, q)$ on $H(M)$ is then defined for $s=(C, b) \in H(p, q), F \in H(M)$ by $F s=(F C) b$.

A local section $M \supset U \stackrel{\sigma}{\rightarrow} H(M)$ determines a frame field $U \ni x \rightarrow q(\sigma(x)) \in C O(M)$ and subspaces of the tangent spaces $T_{q(\sigma(x))}(C O(M))$ which are horizontal for a uniquely defined torsion free connection on the restriction of $C O(M)$ to $U$. The coordinates (2.16) yield in an obvious way bundle coordinates for $(H(M), M, \pi)$.

\subsection{The natural form on $H(M)$}

Let $\nabla$ be a torsion free connection on $C O(M)$. Denote by $\bar{\omega}_{k}{ }_{k}$ the associated connection form and by $F_{\nabla}$ the associated section of $H(M)$ over $C O(M)$. We consider $\left(\bar{\sigma}^{k}, \bar{\omega}_{k}^{i}\right)$, where $\bar{\sigma}^{k}$ is the solder form, as an $\mathbb{R}^{n} \oplus \operatorname{co}(p, q)$-valued form on $C O(M)$. It allows to define a unique $\mathbb{R}^{n} \oplus \operatorname{co}(p, q)$-valued 1-form $\left(\sigma^{k}, \omega^{i}{ }_{k}\right)$ on $H(M)$ by the conditions: (i) it vanishes on tangent vectors which are vertical for the bundle $(H(M), C O(M), q)$, (ii) under the action of $s \in H(p, q)$ it transforms with $\overline{A d}\left(s^{-1}\right)$, and (iii) the pull-back of $\left(\sigma^{k}, \omega_{k}^{i}\right)$ by $F_{\nabla}$ coincides with $\left(\bar{\sigma}^{k}, \bar{\omega}_{k}^{i}\right)$.

On the other hand we have the $\mathbb{R}^{n} \oplus \operatorname{co}(p, q)$-valued solder form $\Sigma$ on the bundle $(H(M), C O(M), q)$, defined at $F \in H(M)$ by $\Sigma(F)=F^{-1} \circ T(q)$. It can be shown from its definition and from the definition of the section $F_{\nabla}$ that it satisfies conditions (i)-(iii) as well. Therefore $\Sigma$ coincides with the form $(\sigma, \omega)$ considered above and it follows that the form $(\sigma, \omega)$ is defined independently of the chosen connection $\nabla$. We call $\Sigma=(\sigma, \omega)$ the "natural form on $H(M)$ ". 
Since the connection $\nabla$ is torsion free, the forms $\bar{\sigma}^{i}, \bar{\omega}^{i}{ }_{j}$ satisfy the first structure equation $\mathrm{d} \bar{\sigma}^{i}=-\bar{\omega}^{i}{ }_{j} \wedge \bar{\sigma}^{j}$ on $C O(M)$. From this ensues that $\Sigma=(\sigma, \omega)$ satisfies

$$
\mathrm{d} \sigma^{i}=-\omega^{i}{ }_{j} \wedge \sigma^{j} \quad \text { on } H(M) .
$$

This follows immediately for vectors tangent to $F_{\nabla}(C O(M))$. Since $\Sigma=(\sigma, \omega)$ vanishes on vectors $Z_{v}$ tangent to the fibres of $(H(M), C O(M), q)$, the term on the right-hand side of the equation vanishes if it is applied to at least one vertical vector field. But it follows immediately from the definition of $\sigma$, that $i_{Z_{v}} \mathrm{~d} \sigma=L_{Z_{v}} \sigma-\mathrm{d}\left(i_{Z_{v}} \sigma\right)=L_{Z_{v}} \sigma=0$ on $H(M)$.

\subsection{The normal conformal Cartan connection on $H(M)$}

A Cartan connection on $(H(M), M, \pi)$ with respect to the groups $H(p, q), C(p, q)$ is given by a $c(p, q)$-valued 1-form $\omega$ on $H(M)$, the "Cartan connection form", satisfying

(1) $\left\langle\omega ; Z_{u}\right\rangle=u$ for the vertical vector field $Z_{u}$ generated by some $u \in h(p, q)$.

(2) $\omega$ is invariant under the adjoint representation and the right action of $H(p, q)$ on $H(M)$, i.e. ${ }^{t} R_{s} \omega=A d\left(s^{-1}\right) \omega$ for any $s \in H(p, q)$, where $R_{s}$ denotes the right action of $s$ on $H(M)$.

(3) $\langle\omega ; X\rangle \neq 0$ for any tangent vector $X \neq 0$.

Using the identification of $c(p, q)$ with $\mathbb{R}^{n} \oplus \operatorname{co}(p, q) \oplus \mathbb{R}^{n *}$, we write $\omega=\left(\sigma^{i}, \omega^{i}{ }_{k}, \omega_{k}\right)$ and consider the "Cartan structure equations"

$$
\begin{aligned}
& \mathrm{d} \sigma^{i}=-\omega^{i}{ }_{k} \wedge \sigma^{k}+\Lambda^{i}, \\
& \mathrm{~d} \omega^{i}{ }_{k}=-\omega^{i}{ }_{l} \wedge \omega_{k}^{l}+S\left(\omega_{p}\right)_{q}{ }_{k}{ }_{k} \wedge \sigma^{q}+{\Omega^{i}}_{k}, \\
& \mathrm{~d} \omega_{k}=-\omega_{i} \wedge \omega^{i}{ }_{k}+\Omega_{k},
\end{aligned}
$$

as defining equations for the "curvature form" $\Omega=\left(\Lambda^{i}, \Omega_{k}^{i}, \Omega_{k}\right)$.

It follows then from the conditions above that the restrictions of the structure equations to the fibres coincide with the Maurer-Cartan equations and that $\left\langle\Omega ; Z_{u} \wedge \cdot\right\rangle=0$ for $u \in h(p, q)$. Thus we have

$$
\Lambda^{i}=\frac{1}{2} T_{k l}^{i} \sigma^{k} \wedge \sigma^{l}, \quad \Omega_{j}^{i}=\frac{1}{2} K_{j k l}^{i} \sigma^{k} \wedge \sigma^{l}, \quad \Omega_{j}=\frac{1}{2} K_{j k l} \sigma^{k} \wedge \sigma^{l}
$$

with certain functions $T^{i}{ }_{k l}, K^{i}{ }_{j k l}, K_{j k l}$ on $H(M)$ such that

$$
T_{k l}^{i}=T_{[k l]}^{i}, \quad K_{j k l}^{i}=K_{j[k l]}^{i}, \quad K_{j k l}=K_{j[k l]} .
$$

The natural form $\Sigma=\left(\sigma^{i}, \omega^{j}{ }_{k}\right)$ can be supplemented by an $\mathbb{R}^{n *}$-valued form $\bar{\omega}_{l}$ on $H(M)$ such that $\left(\sigma^{i}, \omega^{k}{ }_{j}, \bar{\omega}_{l}\right)$ becomes a Cartan connection form. For this purpose we choose a connection $\nabla$ on $C O(M)$ and consider the associated section $F_{\nabla}$ of $H(M)$ over $C O(M)$. A tangent vector $h$ at a point of $F_{\nabla}(C O(M))$ can be decomposed uniquely into a component tangent to $F_{\nabla}(C O(M))$ and a component vertical in $(H(M), C O(M), q)$ which is generated by some $v$ in the $\mathbb{R}^{n *}$-part of $c(p, q)$. We set $\left\langle\bar{\omega}_{j} ; h\right\rangle=v$. The requirement that $\left(\sigma^{i}, \omega^{k}{ }_{j}, \bar{\omega}_{l}\right)$ be invariant under the adjoint representation and the right action of $H(p, q)$ 
on $H(M)$ defines a unique extension of $\bar{\omega}$ to $H(M)$. The resulting form is in fact a Cartan connection form on $H(M)$ for which the Cartan structure equations read

$$
\begin{aligned}
& \mathrm{d} \sigma^{i}=-\omega_{k}^{i} \wedge \sigma^{k}, \\
& \mathrm{~d} \omega_{k}^{i}=-\omega_{l}^{i} \wedge \omega_{k}^{l}+S\left(\bar{\omega}_{p}\right)_{q}{ }_{k}{ }_{k} \wedge \sigma^{q}+\bar{\Omega}_{k}^{i}, \\
& \mathrm{~d} \bar{\omega}_{k}=-\bar{\omega}_{i} \wedge \omega_{k}^{i}+\bar{\Omega}_{k} .
\end{aligned}
$$

We note a few properties of it. It follows from the adjoint action (2.5) of $H(p, q)$ on $c(p, q)$ that $\bar{K}^{i}{ }_{j k l}$ has a tensorial transformation law under the action of $H(p, q)$. Taking the differential of the first of the structure equations and using the other equations to simplify, we get the Bianchi identity

$$
\bar{\Omega}^{i}{ }_{j} \wedge \sigma^{j}=0, \quad \text { i.e. } \bar{K}^{i}{ }_{[j k l]}=0,
$$

which implies in particular

$$
\bar{K}_{i k l}^{i}=-\bar{K}_{l i k}^{i}+\bar{K}_{k i l}^{i} .
$$

As shown by Cartan [3], a specific Cartan connection can be singled out on $H(M)$, the "normal conformal Cartan connection", by requiring the tensor $\bar{K}^{i}{ }_{j k l}$ to be trace free. If $\left(\sigma^{i}, \omega^{i}{ }_{j}, \omega_{j}\right)$ is another Cartan connection form on $H(M)$ with $\left(\sigma^{i}, \omega^{i}{ }_{j}\right)$ the same forms as before, then $\bar{\omega}_{j}-\omega_{j}$ vanishes on vectors vertical in $(H(M), M, \pi)$, whence has an expansion

$$
\bar{\omega}_{j}-\omega_{j}=A_{j k} \sigma^{k}
$$

with some functions $A_{j k}$ on $H(M)$. Subtracting the second structure equations for the two different forms from each other, we get

$$
0=A_{j l} \sigma^{l} \wedge \sigma^{i}+A_{k l} \sigma^{l} \wedge \sigma^{k} \delta^{i}{ }_{j}-\eta^{i k} A_{k l} \sigma^{l} \wedge \sigma^{p} \eta_{p i}+\bar{\Omega}_{j}^{i}-\Omega_{j}^{i}
$$

whence

$$
\begin{aligned}
\bar{K}^{i}{ }_{j r s}-K^{i}{ }_{j r s}= & -A_{j r} \delta^{i}{ }_{s}+A_{j s} \delta^{i}{ }_{r}-\left(A_{s r}-A_{r s}\right) \delta^{i}{ }_{j} \\
& +\eta^{i k}\left(A_{k r} \eta_{s j}-A_{k s} \eta_{r j}\right),
\end{aligned}
$$

from which ensue

$$
\begin{aligned}
& \bar{K}^{i}{ }_{j i s}-K^{i}{ }_{j i s}=(n-2) A_{j s}-\left(A_{s j}-A_{j s}\right)+\eta^{i k} A_{i k} \eta_{s j}, \\
& \bar{K}^{i}{ }_{i r s}-K^{i}{ }_{i r s}=-n\left(A_{s r}-A_{r s}\right), \quad \eta^{j s}\left(\bar{K}^{i}{ }_{j i s}-K^{i}{ }_{j i s}\right)=2(n-1) \eta^{i k} A_{i k} .
\end{aligned}
$$

From these equations follows the requirement that the new Cartan connection satisfies the condition

$$
K^{i}{ }_{j i s}=0
$$


which entails by the Bianchi identity $K^{i}{ }_{i r s}=0$ is equivalent to

$$
A_{j s}=\frac{1}{n-2}\left(\bar{K}_{j i s}^{i}-\frac{1}{n} \bar{K}_{i j s}^{i}-\frac{1}{2(n-1)} \eta^{p q} \bar{K}_{p i q}^{i} \eta_{j s}\right) .
$$

Since the function so defined on $H(M)$ transforms like a tensor under the group $H(p, q)$, we can use it, together with Eq. (2.26), to supplement the natural form on $H(M)$ in a unique way by an $\mathbb{R}^{n *}$-valued form $\omega_{k}$ such that for the resulting Cartan connection, the "normal conformal Cartan connection on $H(M)$ ", the associated tensor field $K^{i}{ }_{j r s}$ satisfies $K^{i}{ }_{j i s}=0$.

We note further properties of it. Besides the first Bianchi identity (2.24) we obtain by taking the differential of the other structure equations the second Bianchi identity

$$
\mathrm{d} \Omega_{j}^{i}=\Omega_{k}^{i} \wedge \omega_{j}^{k}-\omega_{k}^{i} \wedge \Omega_{j}^{k}-S\left(\Omega_{l}\right)_{k}{ }_{j}{ }_{j} \wedge \sigma^{k} \text {. }
$$

Observing that $\Omega^{i}{ }_{i}=0$, we find from this the third Bianchi identity

$$
\Omega_{k} \wedge \sigma^{k}=0 \text { or, equivalently, } K_{[j k l]}=0 .
$$

Furthermore we get the fourth Bianchi identity

$$
\mathrm{d} \Omega_{j}=\Omega_{k} \wedge \omega_{j}^{k}-\omega_{k} \wedge \Omega^{k}{ }_{j}
$$

Finally, we have for $t=(C, b) \in H(p, q)$ the transformation law

$$
A d\left(t^{-1}\right)\left(0, \Omega^{i}{ }_{j}, \Omega_{k}\right)=\left(0, C^{-1 i}{ }_{k} \Omega^{k}{ }_{l} C^{l}{ }_{j},\left(-b_{i} \Omega^{i}{ }_{l}+\Omega_{l}\right) C^{l}{ }_{j}\right) \text {. }
$$

In the bundle coordinates (2.16) the normal conformal Cartan connection form is given explicitly by

$$
\begin{aligned}
\sigma^{i}(x, C, b) & =C^{-1 i}{ }_{k} \sigma^{k}{ }_{\mu} \mathrm{d} x^{\mu}, \\
\omega_{j}^{i}(x, C, b) & =C^{-1 i}{ }_{k}\left(\mathrm{~d} C^{k}{ }_{j}+\Gamma_{p}{ }^{k}{ }_{l} C^{l}{ }_{j} \sigma^{p}{ }_{\mu} \mathrm{d} x^{\mu}\right)+S(b)_{l}{ }_{j} \sigma^{l} \\
& =C^{-1 i}{ }_{k}\left(\mathrm{~d} C^{k}{ }_{j}+\left(\Gamma_{p}{ }^{k}{ }_{l}+S\left(b C^{-1}\right)_{p}{ }_{l}\right) C_{j}^{l}{ }_{j}{ }^{p}{ }_{\mu} \mathrm{d} x^{\mu}\right), \\
\omega_{j}(x, C, b) & =\mathrm{d} b_{j}-b_{k} \omega^{k}{ }_{j}+\frac{1}{2} b_{k} S(b)_{l}{ }_{j}{ }_{j} \sigma^{l}-A_{j k} \sigma^{k}
\end{aligned}
$$

with

$$
A_{j k}=A_{j k}(x, C)=\frac{1}{n-2}\left(\bar{K}_{j i k}^{i}-\frac{1}{n} \bar{K}_{i j k}^{i}-\frac{1}{2(n-1)} \eta^{p q} \bar{K}_{p i q}^{i} \eta_{j k}\right),
$$

where the tensors on the right-hand side are given here by their components with respect to the frame $c_{i}=e_{j} C^{j}{ }_{i}$. For the curvature we get the expressions

$$
\begin{aligned}
C^{i}{ }_{j k l} & \equiv K^{i}{ }_{j k l}=\bar{K}^{i}{ }_{j k l}+2\left\{A_{j l k} \delta_{l]}^{i}-\eta^{i p} A_{p[k} \eta_{l] j}-A_{[k l]} \delta^{i}{ }_{j}\right\}, \\
K_{j k l} & =K_{j k l}(x, C, b)=-b_{i} \bar{K}^{i}{ }_{j k l}-\nabla_{k} A_{j l}+\nabla_{l} A_{j k}+S_{k}{ }^{i}{ }_{j} A_{i l}-S_{l}{ }_{j}{ }_{j} A_{i k} \\
& =-b_{i} C^{i}{ }_{j k l}-\nabla_{k} A_{j l}+\nabla_{l} A_{j k} .
\end{aligned}
$$

The tensor given by (2.36) is the "conformal Weyl tensor". 
In our later applications we shall consider time-orientable conformal structures of Lorentzian signature $(p=1)$ on orientable manifolds. As is natural, we assume then all constructions being restricted to the bundle $C O_{+}^{\uparrow}(M)$ of positively oriented frames with future directed time-like vector. This has structure group $C O_{+}^{\uparrow}(p, q)$, the component of $C O(p, q)$ connected to the unit element. Accordingly we will consider the group $H_{+}^{\uparrow}(p, q)$, the component of the unit element in $H(p, q)$.

\subsection{Change of the section}

Let $U \ni x \rightarrow s_{A}(x) \in \pi^{-1}(U), A=1,2$, denote smooth local sections of $H(M)$ over some open subset $U$ of $M$ and let $U \ni x \rightarrow s(x) \in H(p, q)$ denote the smooth map which satisfies $s_{2}(x)=s_{1}(x) s(x), x \in U$. Denoting by $\omega_{A}=\left(\sigma_{A}{ }^{i}, \omega_{A}{ }^{i}{ }_{k}, \omega_{A j}\right)$ the pull-back of the normal Cartan form with respect to the section $s_{A}$ and by $L_{s}$ the left translation in the group $H(p, q)$, we get the relation

$$
\omega_{2}(x)=A d\left(s(x)^{-1}\right) \omega_{1}(x)+T_{s(x)}\left(L_{s(x)^{-1}}\right) T_{x}(s(\cdot)) .
$$

The special case where $s(x)=(E, f(x)) \in H(p, q)$ with a smooth map $U \ni x \rightarrow$ $f(x) \in \mathbb{R}^{n *}$ gives $T_{s(x)}\left(L_{s(x)^{-1}}\right) T_{x}(s(\cdot)) X=(0,\langle\mathrm{~d} f, X\rangle)$ for any tangent vector $X$ of $U$ and it follows from $(2.38),(2.5)$ that

$$
\begin{aligned}
\left(\sigma_{2}{ }^{i}, \omega_{2}{ }^{i}{ }_{k}, \omega_{2 j}\right)= & \left(\sigma_{1}{ }^{i}, S(f)_{j}{ }^{i}{ }_{k} \sigma_{1}{ }^{j}+\omega_{1}{ }^{i}{ }_{k},-\frac{1}{2} f_{i} S(f)_{j}{ }_{k}{ }_{k} \sigma_{1}{ }^{j}\right. \\
& \left.-f_{i} \omega_{1}{ }^{i}{ }_{k}+\omega_{1 k}+\mathrm{d} f_{k}\right) .
\end{aligned}
$$

Writing

$$
\omega_{A}^{i}{ }_{k}=\Gamma_{A j}{ }_{k}^{i} \sigma^{j}, \quad \omega_{A k}=\Gamma_{A j k} \sigma^{j}, \quad A=1,2,
$$

this gives

$$
\Gamma_{2 j}{ }^{i}{ }_{k}=\Gamma_{1 j}{ }^{i}{ }_{k}+S(f)_{j}{ }_{k}{ }_{k}, \quad \Gamma_{2 j k}=\Gamma_{1 j k}+\nabla_{j} f_{k}-\frac{1}{2} f_{i} S(f)_{j}{ }^{i},
$$

whence also

$$
-A_{2 k j}=-A_{1 k j}+\nabla_{j} f_{k}-\frac{1}{2} f_{i} S(f)_{j}{ }^{i} k
$$

where in the last two equations $\nabla$ denotes the connection defined by $\omega_{1}{ }^{i} k$ respectively $\Gamma_{1 j}{ }^{i} k$.

Let now $g, \tilde{g}$ denote two metrics related by $g=\Omega^{2} \tilde{g}$ and $\nabla, \tilde{\nabla}$ the Levi-Civita connection associated to $g, \tilde{g}$ respectively. We express in the following all connection coefficients, tensor fields, etc. with respect to the same frame field $e_{j}$, which is assumed to be orthonormal with respect to $g$. Let $\Gamma_{i}{ }_{k}{ }_{k}, \tilde{\Gamma}_{i}{ }_{k}{ }_{k}$ denote the connection coefficients with respect to $g$ and $\tilde{g}$ respectively such that $\Gamma_{i}{ }^{j}{ }_{k}=\tilde{\Gamma}_{i}{ }_{k}+S\left(\Omega^{-1} \mathrm{~d} \Omega\right)_{i}{ }_{k}{ }_{k}$ and assume that the connection $\hat{\nabla}$ has connection coefficients given by $\hat{\Gamma}_{i}{ }_{k}=\Gamma_{i}{ }_{k}+S(f)_{i}{ }_{k}$. From the transformation laws given above it follows, that

$$
\tilde{\Gamma}_{j k}=\Gamma_{j k}-\nabla_{j}\left(\Omega^{-1} \nabla_{k} \Omega\right)-\frac{1}{2} \Omega^{-1} \nabla_{i} \Omega S\left(\Omega^{-1} \nabla \Omega\right)_{j}{ }_{k}{ }_{k},
$$




$$
\hat{\Gamma}_{j k}=\Gamma_{j k}+\nabla_{j}\left(f_{k}\right)-\frac{1}{2} f_{i} S(f)_{j}{ }^{i},
$$

whence

$$
\begin{aligned}
\tilde{\Gamma}_{j k} & =\hat{\Gamma}_{j k}-\nabla_{j}\left(f_{k}+\Omega^{-1} \nabla_{k} \Omega\right)+\frac{1}{2}\left\{f_{i} S(f)_{j}{ }^{i}{ }_{k}-\Omega^{-1} \nabla_{i} \Omega S\left(\Omega^{-1} \nabla \Omega\right)_{j}{ }^{i}{ }_{k}\right\} \\
& =\hat{\Gamma}_{j k}-\hat{\nabla}_{j}\left(f_{k}+\Omega^{-1} \nabla_{k} \Omega\right)-\frac{1}{2}\left(f_{i}+\Omega^{-1} \nabla_{i} \Omega\right) S\left(f+\Omega^{-1} \nabla \Omega\right)_{j}{ }^{i}{ }_{k} .
\end{aligned}
$$

\subsection{Conformal geodesics}

With any conformal structure there is associated a distinguished class of curves in the underlying manifold, the "conformal circles", which obey a system of ordinary differential equations of third order [22]. These curves can be obtained, after a reparametrization, as projections of a class of distinguished curves on the bundle $H(M)$, the "conformal geodesics" [14]. We shall in the following discuss the latter, which are particularly useful for us, since they supply in addition to a curve in $M$ a frame field and a connection in the conformal class along that curve.

The normal conformal Cartan connection allows to define for any $z \in \mathbb{R}^{n}$ a smooth "horizontal vector field" $H_{z}$ on $H(M)$ by requiring $\left\langle\left(\sigma^{i}, \omega_{k}^{j}, \omega_{l}\right) ; H_{z}\right\rangle=\left(z^{i}, 0,0\right)$. A conformal geodesic is an integral curve $\gamma(\tau)$ of $H_{z}$. Occasionally we shall also use this name for its projection $\kappa(\tau)=\pi(\gamma(\tau))$ to $M$. In the bundle coordinates (2.16), $\gamma(\tau)$ has the representation $\gamma(\tau)=(x(\tau), C(\tau), b(\tau))$, where

$$
\eta_{i j} C^{i}{ }_{k} C^{j} l=\Theta^{-2} \eta_{k l}
$$

with some $\Theta(\tau)>0$ and $x(\tau)$ is the coordinate representation of $\kappa(\tau)$. The curve $\gamma$ represents a frame field $\left\{c_{k}\right\}_{k=1, \ldots, n}$ along $\pi \gamma$ with $c_{k}=c_{k}^{\mu} \partial_{x^{\mu}}=e_{j} C_{k}^{j}$ and, with respect to this frame, the components $b_{k}=b_{\mu} c_{k}^{\mu}$ of a 1-form field $b_{\mu} \mathrm{d} x^{\mu}$ along $\kappa(\tau)$.

If $\gamma$ is a conformal geodesic as above and $s=(C, f) \in H(p, q)$, we may ask, whether $R_{s}(\gamma)$ is again a conformal geodesic. We find from (2.5) that for arbitrary $z^{i}$

$$
\begin{aligned}
\left\langle\left(\sigma^{i}, \omega^{j}{ }_{k}, \omega_{l}\right) ; \frac{\mathrm{d}}{\mathrm{d} \tau}(\gamma(\tau) s)\right\rangle & =\left\langle\left(\sigma^{i}, \omega^{j}{ }_{k}, \omega_{l}\right) ; T_{\gamma(\tau)}\left(R_{s}\right) H_{z}\right\rangle \\
& =\operatorname{Ad}\left(s^{-1}\right)\left\langle\left(\sigma^{i}, \omega^{j}{ }_{k}, \omega_{l}\right) ; H_{z}\right\rangle \\
& =\operatorname{Ad}\left(s^{-1}\right)\left(z^{i}, 0,0\right)=\left(z^{i}, 0,0\right)
\end{aligned}
$$

for some $z^{i}$ only if $f_{k}=0$ and in that case $z^{i}=C^{-1 i}{ }_{j} z^{j}$, i.e.

$$
T\left(R_{s}\right) H_{z}=H_{C^{-1} z} \quad \text { if } s=(C, 0) \in H(p, q) .
$$

Evaluation of the forms (2.33)-(2.35) at $\mathrm{d}(\gamma(\tau)) / \mathrm{d} \tau$ yields the differential equations

$$
\begin{aligned}
& \frac{\mathrm{d}}{\mathrm{d} \tau} x^{\mu}=e^{\mu}{ }_{i} C^{i}{ }_{j} z^{j}, \\
& \frac{\mathrm{d}}{\mathrm{d} \tau} C^{i}{ }_{j}+\left(\Gamma_{l}{ }_{k}{ }_{k}+S\left(b C^{-1}\right) l^{i}{ }_{k}\right) C^{k}{ }_{j} C^{l}{ }_{m} z^{m}=0,
\end{aligned}
$$




$$
\frac{\mathrm{d}}{\mathrm{d} \tau} b_{j}-\left(\frac{1}{2} b_{k} S(b)_{l}{ }^{k}{ }_{j}+A_{j l}\right) z^{l}=0 .
$$

It should be observed that in these equations tensor $A$ is given with respect to the frame $c_{k}$. Writing $L_{j l}=A_{\mu \nu} e^{\mu}{ }_{j} e^{v}{ }_{l}$ and $d_{j}=b_{\mu} e^{\mu}{ }_{j}$, we have $L_{j l}=A_{i k} C^{-1 i}{ }_{j} C^{-1 k}{ }_{l}, d_{j}=$ $b_{k} C^{-1 k}{ }_{j}$, and the last two equations take the form

$$
\begin{aligned}
& \frac{\mathrm{d}}{\mathrm{d} \tau} C^{i}{ }_{j}+\left(\Gamma_{l}{ }^{i}{ }_{k}+S(d)_{l}{ }^{i}{ }_{k}\right) C^{k}{ }_{j} C^{l}{ }_{m} z^{m}=0, \\
& \frac{\mathrm{d}}{\mathrm{d} \tau} d_{j}-\left(\left(\Gamma_{p}{ }^{k}{ }_{j}+\frac{1}{2} S(d)_{p}{ }^{k}{ }_{j}\right) d_{k}+L_{j p}\right) C^{p}{ }_{q} z^{q}=0 .
\end{aligned}
$$

From these equations follows that $\left(x^{\mu}(\tau), b_{\nu}(\tau)\right)$ satisfy

$$
\begin{aligned}
\left(\nabla_{\dot{x}} \dot{x}\right)^{\mu} & =-S(b)_{\nu}{ }_{\sigma}^{\mu} \dot{x}^{\nu} \dot{x}^{\sigma}, \\
\left(\nabla_{\dot{x}} b\right)_{\mu} & =\left(\frac{1}{2} b_{\sigma} S(b)_{\nu}{ }^{\sigma}{ }_{\mu}+L_{\nu \mu}\right) \dot{x}^{\nu},
\end{aligned}
$$

where the dot denotes differentiation with respect to $\tau$. With an obvious meaning of the notation we write Eqs. (2.47), (2.48) in the short form:

$$
\begin{aligned}
& \nabla_{\dot{x}} \dot{x}=-2\langle b, \dot{x}\rangle \dot{x}+(\dot{x}, \dot{x}) b, \\
& \nabla_{\dot{x}} b=\langle b, \dot{x}\rangle b-\frac{1}{2}(b, b) \dot{x}+L(\dot{x}, \cdot) .
\end{aligned}
$$

In a similar way we find that the frame vector fields $c_{k}$ satisfy the equations

$$
\nabla_{\dot{x}} c_{k}=-\left\langle b, c_{k}\right\rangle \dot{x}-\langle b, \dot{x}\rangle c_{k}+\left(c_{k}, \dot{x}\right) b .
$$

Here indices are moved with the fixed metric in the conformal class which is indicated in the equations by $(\cdot, \cdot)$. It should be clear from the context where the covariant or the contravariant form of $b$ or $\dot{x}$ appears on the right-hand sides of the equations.

\section{Conformal Einstein spaces}

Most results of this section could be stated, as in Lemma 3.1, for Einstein spaces of arbitrary signature and dimension $n \geq 3$. To avoid lengthy distinctions between various possible cases and since we want to employ the two-component spinor calculus later on, we restrict our discussion mainly to the four-dimensional Lorentz case.

\subsection{The conformal Einstein equations}

Suppose the Lorentz metric $\tilde{g}$ on the oriented four-dimensional manifold $\tilde{M}$ admits a time orientation and is a smooth solution to the Einstein equations

$$
\tilde{R}_{\mu \nu}=\lambda \tilde{g}_{\mu \nu}
$$


with cosmological constant $\lambda$. For a given positive, smooth function $\Omega$ on $\tilde{M}$, consider the rescaled metric

$$
g=\Omega^{2} \tilde{g},
$$

its associated Levi-Civita connection $\nabla$, and the fields

$$
\begin{aligned}
& \bar{s}=\frac{1}{4} \nabla_{\mu} \nabla^{\mu} \Omega+\frac{1}{24} R \Omega, \quad L_{\mu \nu}=\frac{1}{2}\left(R_{\mu \nu}-\frac{1}{6} R g_{\mu \nu}\right), \\
& d^{\mu}{ }_{\nu \lambda \rho}=\Omega^{-1} C^{\mu}{ }_{\nu \lambda \rho},
\end{aligned}
$$

where $R, R_{\mu \nu}, C^{\mu \lambda \rho}$ denote the Ricci scalar, the Ricci tensor, and the Weyl tensor of $g$ respectively. The Einstein equations for $\tilde{g}$ are equivalent to the equations

$$
\begin{aligned}
& \nabla_{\mu} \nabla_{\nu} \Omega=-\Omega L_{\mu \nu}+\bar{s} g_{\mu \nu}, \\
& \nabla_{\mu} \bar{s}=-L_{\mu \nu} \nabla^{\nu} \Omega, \\
& \nabla_{\lambda} L_{\rho \nu}-\nabla_{\rho} L_{\lambda \nu}=\nabla_{\mu} \Omega d^{\mu}{ }_{\nu \lambda \rho}, \\
& \nabla_{\mu} d^{\mu}{ }_{\nu \lambda \rho}=0, \\
& 6 \Omega \bar{s}-3 \nabla_{\mu} \Omega \nabla^{\mu} \Omega=\lambda .
\end{aligned}
$$

The derivation and discussion of these equations and their application to the study of certain classes of solutions to the Einstein equations at large can be found in [7] and the references given therein. We may think of them as being obtained from the Einstein equations by introducing an artificial gauge freedom corresponding to the rescalings (3.2).

Although we shall in the following consider a somewhat different conformal representation of the Einstein equations, we note for later use the constraint equations (cf. [6]) induced by the equations above on a hypersurface $S$ with normal vector field $n$ satisfying $g(n, n)=$ $\varepsilon= \pm 1$. For this purpose we express the equations with respect to a frame $c_{k}, k=0,1,2,3$, defined near $S$ which is orthonormal with respect to $g$. If $S$ is space-like ( $\varepsilon=1)$ we assume that $c_{0}=n$, that indices $a, b, c, \ldots$ take values $1,2,3$, and that $n$, if used as andex, takes value 0 . If $S$ is time-like $(\varepsilon=-1)$ we assume that $c_{3}=n$, that indices $a, b, c, \ldots$ take values $0,1,2$, and that $n$, if used as an index, takes value 3 . Orthogonal projections of tensors into the hypersurface $S$ are then given by their components with respect to the interior frame $c_{a}$. We write in particular

$$
L_{a}=L_{a n}, \quad d_{a b}=d_{a n b n}, \quad d_{a b c}=d_{a n b c},
$$

respectively for the projections of the fields

$$
L_{\mu \nu} n^{\nu}, \quad d_{\mu \nu \lambda \rho} n^{\nu} n^{\rho}, \quad d_{\mu \nu \lambda \rho} n^{\nu}
$$

into $S$ and set $\Sigma=n(\Omega)$. The tensor field $d_{a b}$ is called the " $n$-electric" part and $d_{a b}^{*} \equiv$ $d_{a n b n}^{*}=-\frac{1}{2} d_{a c d} \varepsilon_{b}{ }^{c d}$ the " $n$-magnetic" part of the tensor $d_{i j k l}$. Here the star denotes the four-dimensional dual and $\varepsilon_{a b c}$ the totally symmetric Levi-Civita symbol which takes the value 1 if the indices are different and in the natural order. Then, 


$$
\begin{aligned}
& d_{a b}=d_{b a}, \quad d_{a}^{a}=0, \quad d_{a b c}=-d_{a c b}, \quad d_{[a b c]}=0 \\
& d_{a b c d}=2\left\{h_{a[c} d_{d] b}+h_{b[d} d_{c] a}\right\} .
\end{aligned}
$$

We define the four-dimensional connection coefficients with respect to the frame $c_{k}$ by $\nabla_{c_{i}} c_{j}=\Gamma_{i}{ }^{k}{ }_{j} c_{k}$ and assume that $\nabla_{n} c_{k}=0$ near $S$. Denoting the Levi-Civita connection of the induced metric $h$ on $S$ by $D$, we have $D_{c_{a}} c_{b}=\Gamma_{a}{ }^{d}{ }_{b} c_{d}$ on $S$, and the second fundamental form of $S$ is given by

$$
\chi_{a b}=g\left(\nabla_{c_{a}} n, c_{b}\right)=\Gamma_{a}{ }_{n}^{j} \eta_{j b}=-\Gamma_{a}^{j}{ }_{b} \eta_{j n} .
$$

The constraint equations take the form

$$
\begin{aligned}
& D_{a} D_{b} \Omega=-\varepsilon \Sigma \chi_{a b}-\Omega L_{a b}+\bar{s} h_{a b}, \\
& D_{a} \Sigma=\varepsilon \chi_{a}{ }^{c} D_{c} \Omega-\Omega L_{a}, \\
& D_{a} \bar{s}=-D^{b} \Omega L_{b a}-\varepsilon \Sigma L_{a}, \\
& D_{a} L_{b c}-D_{b} L_{a c}=D^{e} \Omega d_{e c a b}-\varepsilon \Sigma d_{c a b}-\varepsilon\left(\chi_{a c} L_{b}-\chi_{b c} L_{a}\right), \\
& D_{a} L_{b}-D_{b} L_{a}=D^{e} \Omega d_{e a b}+\chi_{a}{ }^{c} L_{b c}-\chi_{b}{ }^{c} L_{a c}, \\
& D^{c} d_{c a b}=\varepsilon\left(\chi^{c}{ }_{a} d_{b c}-\chi^{c}{ }_{b} d_{a c}\right), \\
& D^{a} d_{a b}=\chi^{a c} d_{a b c}, \\
& D_{b} \chi_{c a}-D_{c} \chi_{b a}=\Omega d_{a b c}+h_{a b} L_{c}-h_{a c} L_{b}, \\
& l_{a b}=\Omega d_{a b}+L_{a b}+\varepsilon\left(\chi_{c}{ }^{c}\left(\chi_{a b}-\frac{1}{4} \chi_{d}{ }^{d} h_{a b}\right)-\chi_{c a} \chi_{b}{ }^{c}+\frac{1}{4} \chi_{c d} \chi^{c d} h_{a b}\right\}, \\
& \lambda=6 \Omega \bar{s}-3 \varepsilon \Sigma^{2}-3 D_{a} \Omega D^{a} \Omega .
\end{aligned}
$$

To obtain the constraint equations we use the decomposition

$$
R_{i j k l}=\Omega d_{i j k l}+g_{i k} L_{l j}-g_{i l} L_{k j}+L_{i k} g_{l j}-L_{i l} g_{k j},
$$

of the curvature tensor $R_{i j k l}$ of $g$, the decomposition

$$
r_{a b c d}=2\left\{h_{a[c} l_{d] b}+h_{b[d} l_{c] a}\right\}
$$

of the curvature tensor $r_{a b c d}$ of $h$, where $l_{a b}=r_{a b}-\frac{1}{4} r h_{a b}$ and $r_{a b}, r$ denote the Ricci tensor and scalar respectively, and finally the Gauss equation and the Codazzi equation

$$
R_{a b c d}=r_{a b c d}+\chi_{a c} \chi_{b d}-\chi_{a d} \chi_{b c}, \quad R_{a n b c}=D_{b} \chi_{c a}-D_{c} \chi_{b a} .
$$

Another conformal representation of the Einstein equations will now be derived. The Levi-Civita connection $\tilde{\nabla}$ of the solution $\tilde{g}$ to the Einstein equation (1.1) and a frame which is orthonormal for $\tilde{g}$ define a section of the bundle $H(\tilde{M})$ associated with $\tilde{g}$. The new representation of the Einstein equations is obtained by expressing the structure equations as well as the Bianchi identity (2.29) with respect to an arbitrary section of $H(\tilde{M})$. The latter 
is given by a frame $c_{k}, k=0,1,2,3$, which is orthonormal for the arbitrary metric $g$ in the conformal class of $\tilde{g}$ and by a connection $\hat{\nabla}$ which is conformal for $\tilde{g}$.

We write $\nabla, \tilde{\nabla}$ for the Levi-Civita connections of $g, \tilde{g}$ respectively and denote the 1 -forms dual to $c_{k}$, which satisfy (2.9), by $\sigma^{j}$. The connection coefficients of $\nabla, \tilde{\nabla}$, and $\hat{\nabla}$ in the frame $c_{k}$ are denoted by $\Gamma_{i}{ }^{j}{ }_{k}, \tilde{\Gamma}_{i}^{j}{ }_{k}, \hat{\Gamma}_{i}{ }_{k}{ }_{k}$ respectively. For the difference tensors defined by the connections we have in short notation

$$
\hat{\nabla}-\tilde{\nabla}=S(b), \quad \hat{\nabla}-\nabla=S(f)
$$

and in index notation

$$
\begin{aligned}
& \hat{\Gamma}_{i}{ }^{j}{ }_{k}-\tilde{\Gamma}_{i}{ }^{j}{ }_{k}=\delta^{j}{ }_{i} b_{k}+\delta^{j}{ }_{k} b_{i}-\eta_{i k} \eta^{j l} b_{l}, \\
& \hat{\Gamma}_{i}{ }^{j}{ }_{k}-\Gamma_{i}{ }^{j}{ }_{k}=\delta^{j}{ }_{i} f_{k}+\delta^{j}{ }_{k} f_{i}-\eta_{i k} \eta^{j l} f_{l}
\end{aligned}
$$

with 1-forms $b=b_{k} \sigma^{k}$ and $f=f_{k} \sigma^{k}$ on $\tilde{M}$ such that

$$
f_{\mu}=b_{\mu}-\Omega^{-1} \nabla_{\mu} \Omega
$$

and

$$
f_{k}=f_{\mu} c_{k}^{\mu}=\frac{1}{4} \hat{\Gamma}_{k}^{j}{ }_{j}
$$

We shall now consider the normal conformal Cartan connection on the bundle $H(\tilde{M})$. The pull-back to $\tilde{M}$ of the curvature form $\Omega_{j}$ by the section defined by the connection $\tilde{\nabla}$ and the frame $c_{k}$ vanishes by (2.37) because $\tilde{g}$ satisfies the Einstein equation (3.1). Using instead the section defined by the connection $\hat{\nabla}$ and the frame $c_{k}$, we get from the transformation law (2.32), that the resulting tensor $K_{i j k}$ is given by

$$
K_{j k l}=-d_{i} d^{i}{ }_{j k l}
$$

with

$$
d_{k}=\Omega b_{k}
$$

and

$$
d^{i}{ }_{j k l}=\Omega^{-1} C^{i}{ }_{j k l},
$$

where $C^{i}{ }_{j k l}$ is the conformal Weyl tensor of $g$.

The Cartan connection form and the forms determined by it on $H(\tilde{M})$ are pulled back now to $\tilde{M}$ with the section defined by the connection $\hat{\nabla}$ and the frame $c_{k}$. The forms obtained on $\tilde{M}$ will be denoted by the same symbol as the original forms on $H(\tilde{M})$. We write $\omega^{i}{ }_{j}=\hat{\Gamma}_{k}{ }_{j}{ }_{j} \sigma^{k}, \omega_{j}=\hat{\Gamma}_{k j} \sigma^{k}$ and $\hat{\nabla}_{k}=\hat{\nabla}_{c_{k}}$, etc. The structure equations take the form

$$
\Sigma_{p}^{i}{ }_{q}=0, \quad \Delta_{k p q}^{i}=0, \quad \Delta_{p q}=0, \quad \Delta_{k p q}=0,
$$

where

$$
\Sigma_{p}{ }^{i}{ }_{q} c_{i} \equiv\left\langle\Sigma^{i} ; c_{p} \wedge c_{q}\right\rangle c_{i}=\left(\hat{\Gamma}_{p}{ }^{l}{ }_{q}-\hat{\Gamma}_{q}{ }^{l}{ }_{p}\right) c_{l}-\left[c_{p}, c_{q}\right]
$$




$$
\begin{aligned}
\Delta^{i}{ }_{j p q} \equiv & \left\langle\Delta^{i}{ }_{j} ; c_{p} \wedge c_{q}\right\rangle=c_{p}\left(\hat{\Gamma}_{q}{ }^{i}{ }_{j}\right)-c_{q}\left(\hat{\Gamma}_{p}{ }^{i}{ }_{j}\right)-\hat{\Gamma}_{k}{ }^{i}{ }_{j}\left(\hat{\Gamma}_{p}{ }^{k}{ }_{q}-\hat{\Gamma}_{q}{ }^{k}{ }_{p}\right) \\
& +\hat{\Gamma}_{p}{ }^{i}{ }_{k} \hat{\Gamma}_{q}{ }^{k}{ }_{j}-\hat{\Gamma}_{q}{ }^{i}{ }_{k} \hat{\Gamma}_{p}{ }^{k}{ }_{j}-\delta^{i}{ }_{q} \hat{\Gamma}_{p j}+\delta^{i}{ }_{p} \hat{\Gamma}_{q j}-\delta^{i}{ }_{j}\left(\hat{\Gamma}_{p q}-\hat{\Gamma}_{q p}\right) \\
& +\eta^{i k}\left(\hat{\Gamma}_{p k} \eta_{q j}-\hat{\Gamma}_{q k} \eta_{p j}\right)-\Omega d^{i}{ }_{j p q}, \\
\Delta_{p q} \equiv & \frac{1}{4}\left\langle\Delta^{i}{ }_{i} ; c_{p} \wedge c_{q}\right\rangle=\hat{\nabla}_{p} f_{q}-\hat{\nabla}_{q} f_{p}-\hat{\Gamma}_{p q}+\hat{\Gamma}_{q p}, \\
\Delta_{k p q} \equiv & \left\langle\Delta_{k}, c_{p} \wedge c_{q}\right\rangle=\hat{\nabla}_{p} \hat{\Gamma}_{q j}-\hat{\nabla}_{q} \hat{\Gamma}_{p j}+d_{i} d^{i}{ }_{j p q} .
\end{aligned}
$$

Using (3.19), (3.21)-(3.23) we can express the Bianchi identity (2.29) in the form

$$
\hat{\nabla}_{i} d^{i}{ }_{j k l}=f_{i} d^{i}{ }_{j k l}
$$

or, equivalently,

$$
\nabla_{i} d^{i}{ }_{j k l}=0 .
$$

The coupled system of Eqs. (3.24), (3.29) respectively (3.30) gives the new conformal representation of the Einstein equations. For the rest of the article we shall only refer to this system, or to the system obtained from it by using (3.19) to replace $\hat{\Gamma}_{q}{ }^{i}{ }_{j}$ by $\Gamma_{q}{ }^{i}{ }_{j}$ and $f_{k}$, as to the "conformal Einstein equations". The fields $\Omega$ and $d_{j}$, whose occurrence in the field equations reflects the artificial gauge freedom are, of course, not governed by a field equation. They will be taken care of later by our gauge conditions. The relationship of the resulting equations with the Einstein equations (3.1) and with the conformal representation of the Einstein equations used in our previous work can be traced in detail in our discussion of the evolution of the constraints in Section 6.

\subsection{The conformal field equations in spinor form}

In the following the two-component spinor calculus will be used. We quickly review our conventions, following in most parts those in [7]. For a detailed account of spinor techniques we refer to [18]. Indices $a, b, c, \ldots a^{\prime}, b^{\prime}, c^{\prime}, \ldots$, take values 0,1 and the summation rule is implied. The basic antisymmetric spinors, which are used to lower or lift indices, are given by $\varepsilon_{a b}, \varepsilon^{a b}$ with $\varepsilon_{01}=\varepsilon^{01}=1$. It follows that $\varepsilon_{b a} \varepsilon^{b c}=\varepsilon_{a}^{c}$ is a Kronecker delta symbol. Analogous rules hold for the primed $\varepsilon$-spinors. Their invariance group is given by

$$
S L(2, C)=\left\{t_{b}^{a} \in G L(2, C) \mid \varepsilon_{a b} t^{a}{ }_{c} t^{b}{ }_{d}=\varepsilon_{c d}\right\} .
$$

The constant van der Waerden symbols $\sigma_{j}{ }^{a a^{\prime}}, \sigma^{k} b b^{\prime}$ are defined by the maps

$$
\begin{aligned}
& \mathbb{R}^{4} \ni\left(x^{j}\right) \rightarrow x^{a a^{\prime}}=x^{j} \sigma_{j}{ }^{a a^{\prime}}=\frac{1}{\sqrt{2}}\left(\begin{array}{cc}
x^{0}+x^{3} & x^{1}+i x^{2} \\
x^{1}-i x^{2} & x^{0}-x^{3}
\end{array}\right), \\
& \mathbb{R}^{4 *} \ni\left(\xi_{j}\right) \rightarrow \xi_{a a^{\prime}}=\xi_{j} \sigma_{a a^{\prime}}^{j}=\frac{1}{\sqrt{2}}\left(\begin{array}{cc}
\xi_{0}+\xi_{3} & \xi_{1}-i \xi_{2} \\
\xi_{1}+i \xi_{2} & \xi_{0}-\xi_{3}
\end{array}\right),
\end{aligned}
$$

which identify $\mathbb{R}^{4}$ respectively $\mathbb{R}^{4 *}$ with hermitian $2 \times 2$ matrices. Since the van der Waerden symbols satisfy 


$$
\delta_{k}^{j}=\sigma_{a a^{\prime}}^{j} \sigma_{k}^{a a^{\prime}}, \quad \varepsilon_{a}^{b} \varepsilon_{a^{\prime}}^{b^{\prime}}=\sigma_{a a^{\prime}}^{j} \sigma_{j}^{b b^{\prime}}, \quad \eta_{j k} \sigma_{a a^{\prime}}^{j} \sigma_{b b^{\prime}}^{k}=\varepsilon_{a b} \varepsilon_{a^{\prime} b^{\prime}},
$$

the $2: 1$ homomorphism of the simply connected group $\mathbb{R}^{+} \times S L(2, C)$ onto the product $C O_{+}^{\uparrow}(1,3)=\mathbb{R}^{+} \times O_{+}^{\uparrow}(1,3)$ of the group of positive real numbers with the component of the Lorentz group connected to the unit element is realized by

$$
\mathbb{R}^{+} \times S L(2, C) \ni\left(\lambda, t^{a}{ }_{b}\right) \cong \lambda t^{a}{ }_{b} \stackrel{\Phi}{\rightarrow} t^{i}{ }_{j}=\lambda^{2} \sigma^{i}{ }_{a a^{\prime}} t^{a}{ }_{b} \bar{t}^{a^{\prime}}{ }_{b^{\prime}} \sigma_{j}{ }^{b b^{\prime}} \in C O_{+}^{\uparrow}(1,3) .
$$

This map induces an isomorphism of Lie algebras

$$
\begin{aligned}
\mathbb{R} \oplus \operatorname{sl}(2, C) \ni v^{a}{ }_{b} & =\lambda \varepsilon_{b}{ }^{a}+u^{a}{ }_{b} \stackrel{\Phi_{*}}{\rightarrow} v^{i}{ }_{j} \\
& =\sigma^{i}{ }_{a a^{\prime}} v^{a}{ }_{b} \sigma_{j}{ }^{b a^{\prime}}+\sigma^{i}{ }_{a a^{\prime}} \bar{v}^{a^{\prime}}{ }_{b^{\prime}} \sigma_{j}{ }^{a b^{\prime}} \in \operatorname{co}(1,3)
\end{aligned}
$$

with inverse

$$
\operatorname{co}(1,3) \ni v_{j}^{i} \stackrel{\Phi_{*}^{-1}}{\rightarrow} v^{a}{ }_{b}=\frac{1}{2} v_{j}^{i}\left(\sigma_{i}{ }^{a c^{\prime}} \sigma^{j}{ }_{b c^{\prime}}-\frac{1}{4} \sigma_{i}{ }^{c c^{\prime}} \sigma^{j}{ }_{c c^{\prime}} \varepsilon_{b}{ }^{a}\right) \in \mathbb{R} \oplus \operatorname{sl}(2, C) .
$$

Restricting to $\lambda=1$, i.e. to the group $S L(2, C)$, we obtain from $\Phi$ a $2: 1$ homomorphism of $\operatorname{SL}(2, C)$ onto $O_{+}^{\uparrow}(1,3)$. We denote this map by $\Phi^{\prime}$ and the induced isomorphism of the Lie algebra $s l(2, C)$ onto the Lie algebra $o(1,3)$ by $\Phi_{*}^{\prime}$.

Suppose $g$ is a metric in the conformal class $\mathcal{C}$. If $e_{k}$ is a frame field which is orthonormal with respect to $g$ and $\alpha^{j}$ the dual 1 -form field, we associate with them the frame $e_{a a^{\prime}}=$ $e_{k} \sigma^{k} b b^{\prime}$ and the 1-form field $\alpha^{a a^{\prime}}=\alpha^{j} \sigma_{j}{ }^{a a^{\prime}}$. The duality and normalization conditions then take the form

$$
\left\langle\alpha^{b b^{\prime}}, e_{a a^{\prime}}\right\rangle=\varepsilon_{a}^{b} \varepsilon_{a^{\prime}}^{b^{\prime}}, \quad g\left(e_{a a^{\prime}}, e_{b b^{\prime}}\right)=\varepsilon_{a b} \varepsilon_{a^{\prime} b^{\prime}} .
$$

It will be assumed that there exists a principal fibre bundle $S L(\tilde{M}) \stackrel{\pi^{\prime}}{\rightarrow} \tilde{M}$ with structure group $S L(2, C)$ which provides a twofold covering $S L(\tilde{M}) \stackrel{\psi^{\prime}}{\rightarrow} O_{+}^{\uparrow}(\tilde{M})$ of the bundle of positively oriented and time oriented $g$-orthonormal frames which is a morphism of principal fibre bundles with respect to the homomorphism $\Phi^{\prime}$. The question of the existence of such a spin structure poses no problem for us, since in the situations studied in this article there exist globally defined orthonormal frames. Also, it is irrelevant for our work which spin structure is chosen if there exist more than one.

We consider $S L(\tilde{M})$ in the following as the set of spin frames $\delta=\left(\delta_{a}\right)_{a=0,1}$ at points of $\tilde{M}$ which are normalized by $\varepsilon\left(\delta_{a}, \delta_{b}\right)=\varepsilon_{a b}$ and the action of $t \in S L(2, C)$ on $\delta \in S L(\tilde{M})$ as being defined by $\delta \cdot t=\left(\delta_{b} t_{a}^{b}\right)_{a=0,1}$. Spinors $\kappa, \mu$ at a point $p \in \tilde{S}$ define a pair of complex conjugate null vectors at $p$ one of which we write $\kappa \bar{\mu}$. Therefore any $\delta \in S L(\tilde{M})$ determines a frame $e_{a a^{\prime}}=\delta_{a} \bar{\delta}_{a^{\prime}}$ satisfying the normalization condition given above. We can assume that the map $\psi^{\prime}$ is given by $\delta \rightarrow\left(e_{k}=\delta_{a} \bar{\delta}_{a^{\prime}} \sigma_{k} a a^{\prime}\right)_{k=0,1,2,3}$.

In an obvious way the map $\psi^{\prime}$ can be extended to obtain the twofold covering $C S L(\tilde{M}) \stackrel{\psi}{\rightarrow}$ $C O_{+}^{\uparrow}(\tilde{M})$ of the positively oriented and time oriented $g$-conformal frames by the principal fibre bundle with structure group $\mathbb{R}^{+} \times S L(2, C)$ and bundle space given by the set $C S L(\tilde{M})$ of spin frames satisfying $\varepsilon\left(\delta_{a}, \delta_{b}\right)=\lambda^{2} \varepsilon_{a b}$ with some $\lambda \in \mathbb{R}^{+}$. 
Any spinor field on $\tilde{M}$ induces a function on $\operatorname{CSL}(\tilde{M})$ whose values at a point $\delta \in$ $C S L(\tilde{M})$ is given by the components of the spinor field with respect to the spin frame $\delta$. Under the action of the group on the fibres, this function transforms under a representation of the group determined by the index structure of the spinor field. We shall call such functions "invariant".

If $\psi^{\prime}$ is used to pull-back the connection form which represents the unique torsion free connection $\nabla$ on $O_{+}^{\uparrow}(\tilde{M})$ and the map $\Phi_{*}^{\prime-1}$ is applied, we obtain an $s l(2, C)$-valued connection form on $S L(\tilde{M})$ which will be called the Levi-Civita connection form on $S L(\tilde{M})$ and denoted by $\omega^{a}{ }_{b}$, a notation maintained for its canonical extension to $C S L(\tilde{M})$. In a similar way any connection form on $C O_{+}^{\uparrow}(\tilde{M})$ representing a torsion free connection $\hat{\nabla}$ can be pulled back by $\psi$ and combined with $\Phi_{*}^{-1}$ to obtain an $\mathbb{R} \oplus \operatorname{sl}(2, C)$-valued connection form $\hat{\omega}^{a}{ }_{b}$ on $C S L(\tilde{M})$. Finally we pull-back to $C S L(\tilde{M})$ the $\mathbb{R}^{4}$-valued solder form on $C O_{+}^{\uparrow}(\tilde{M})$, transvect it with the appropriate van der Waerden symbol and denote the resulting form by $\sigma^{a a^{\prime}}$. The 1-form $f$ which relates by (3.19) the connection $\nabla$ to $\hat{\nabla}$, implies an invariant function on $C S L(\tilde{M})$, which we write as $f_{a a^{\prime}}$. Eq. (3.19) takes on $C S L(\tilde{M})$ the form

$$
\hat{\omega}^{a}{ }_{b}=\omega^{a}{ }_{b}+f_{b c^{\prime}} \sigma^{a c^{\prime}} .
$$

We shall express now the conformal Einstein equations as a system of equations for forms on $C S L(\tilde{M})$. The function $\Omega$ pulls back to an invariant function on $C S L(\tilde{M})$, denoted by the same symbol, which is constant on the fibres. The 1 -form $d_{k}$ and the tensor field $\hat{\Gamma}_{i j}$ on $\tilde{M}$ are represented by invariant functions on $C S L(\tilde{M})$ which we write $d_{a a^{\prime}}$ respectively $\Phi_{c c^{\prime} a a^{\prime}}$. The conformal Weyl tensor is represented by a completely symmetric spinor field which induces an invariant function denoted by $\phi_{a b c d}$. We set

$$
K_{a a^{\prime} b b^{\prime} c c^{\prime}}=-d^{e e^{\prime}}\left(\phi_{e a b c} \varepsilon_{e^{\prime} a^{\prime}} \varepsilon_{b^{\prime} c^{\prime}}+\bar{\phi}_{e^{\prime} a^{\prime} b^{\prime} c^{\prime}} \varepsilon_{e a} \varepsilon_{b c}\right) .
$$

It will be convenient to express the conformal field equations in terms of the forms

$$
\begin{aligned}
& \Omega, \quad \sigma^{a a^{\prime}}, \quad \hat{\omega}_{b}^{a}, \quad \hat{\omega}=\hat{\omega}_{a}^{a}=f_{a a^{\prime}} \sigma^{a a^{\prime}}, \quad \omega_{b}^{a}, \quad \hat{\omega}_{a a^{\prime}}=\Phi_{c c^{\prime} a a^{\prime}} \sigma^{c c^{\prime}}, \\
& \Omega_{b}^{a}{ }_{b}=-\frac{1}{2} \phi_{b c d}^{a} \sigma_{d^{\prime}} \wedge \sigma^{d d^{\prime}}, \quad \Omega_{a a^{\prime}}=\frac{1}{2} K_{a a^{\prime} b b^{\prime} c c^{\prime}} \sigma^{b b^{\prime}} \wedge \sigma^{c c^{\prime}},
\end{aligned}
$$

where the relation (3.32) is assumed. With the notation

$$
\begin{aligned}
& \Sigma^{a a^{\prime}}=\mathrm{d} \sigma^{a a^{\prime}}+\omega^{a}{ }_{b} \wedge \sigma^{b a^{\prime}}+\bar{\omega}^{a^{\prime}}{ }_{b^{\prime}} \wedge \sigma^{a b^{\prime}}, \\
& \Delta^{a}{ }_{b}=\mathrm{d} \hat{\omega}^{a}{ }_{b}+\hat{\omega}^{a}{ }_{c} \wedge \hat{\omega}^{c}{ }_{b}-\hat{\omega}_{b c^{\prime}} \wedge \sigma^{a c^{\prime}}-\Omega \Omega^{a}{ }_{b}, \\
& \Delta=\Delta^{a}{ }_{a}=\mathrm{d} \hat{\omega}-\hat{\omega}_{c c^{\prime}} \wedge \sigma^{c c^{\prime}}, \\
& \Delta_{a a^{\prime}}=\mathrm{d} \hat{\omega}_{a a^{\prime}}+\hat{\omega}_{a c^{\prime}} \wedge \overline{\hat{\omega}}^{c^{\prime}}{ }_{a^{\prime}}+\hat{\omega}_{c a^{\prime}} \wedge \hat{\omega}^{c}{ }_{a}-\Omega_{a a^{\prime}}, \\
& \Lambda_{b}^{a}=\mathrm{d} \Omega^{a}{ }_{b}-\Omega^{a}{ }_{c} \wedge \omega^{c}{ }_{b}+\omega^{a}{ }_{c} \wedge \Omega^{c}{ }_{b},
\end{aligned}
$$

where it should be observed that in the first and the last equation the connection form defined by $\nabla$ occurs, the conformal Einstein equations take the form

$$
\Sigma^{a a^{\prime}}=0, \quad \Delta_{b}^{a}=0, \quad \Delta=0, \quad \Delta_{a a^{\prime}}=0, \quad \Lambda_{b}^{a}=0 .
$$


Although the third of these equations is in fact the contraction of the second we added it to the list for later convenience. It may also be noted that in view of (3.32) the first equation still holds if in the expression for $\Sigma^{a a^{\prime}}$ the connection form $\omega^{a}{ }_{b}$ is replaced by $\hat{\omega}^{a}{ }_{b}$.

On $\tilde{M}$ a representation of the conformal Einstein equations is obtained by taking the pull-backs of Eqs. (3.33) with respect to a local section of $S L(\tilde{M})$, considered as subbundle of $C S L(\tilde{M})$. The section defines a spin frame field $\delta_{a}$ whence the frame field $c_{a a^{\prime}}=\delta_{a} \bar{\delta}_{a^{\prime}}$ satisfying $g\left(c_{a a^{\prime}}, c_{b b^{\prime}}\right)=\varepsilon_{a b} \varepsilon_{a^{\prime} b^{\prime}}$. The pull-back of the solder form gives the 1-form field dual to $c_{a a^{\prime}}$, which we denote again by $\sigma^{a a^{\prime}}$. The pull-backs of the connection forms can then be written as

$$
\omega_{b}^{a}=\Gamma_{c c^{\prime}}{ }_{b} \sigma^{c c^{\prime}}, \quad \hat{\omega}^{a}{ }_{b}=\hat{\Gamma}_{c c^{\prime}}{ }^{a}{ }_{b} \sigma^{c c^{\prime}}
$$

with connection coefficients satisfying

$$
\Gamma_{c c^{\prime} a b}=\Gamma_{c c^{\prime} b a}, \quad \hat{\Gamma}_{c c^{\prime}}{ }^{a}{ }_{b}=\Gamma_{c c^{\prime}}{ }^{a}{ }_{b}+\varepsilon_{c}{ }^{a} f_{b c^{\prime}}
$$

The connection coefficients used in calculations with tensors are given by

$$
\begin{aligned}
& \Gamma_{b b^{\prime}}{ }^{a a^{\prime}}{ }_{c c^{\prime}}=\Gamma_{b b^{\prime}}{ }^{a}{ }_{c} \varepsilon_{c^{\prime}}{ }^{a^{\prime}}+\bar{\Gamma}_{b b^{\prime}}{ }^{a^{\prime}}{ }_{c^{\prime}} \varepsilon_{c}{ }^{a}, \\
& \hat{\Gamma}_{b b^{\prime}}{ }^{a a^{\prime}}{ }_{c c^{\prime}}=\hat{\Gamma}_{b b^{\prime}}{ }^{a}{ }_{c} \varepsilon_{c^{\prime}}{ }^{a^{\prime}}+\overline{\hat{\Gamma}}_{b b^{\prime}}{ }^{a^{\prime}}{ }_{c^{\prime}} \varepsilon_{c}{ }^{a} .
\end{aligned}
$$

Evaluation of the pull-backs of the other forms with repect to $c_{a a^{\prime}}$ yields the conformal field equations in the form

$$
\begin{aligned}
& 0=\Sigma_{b b^{\prime}}{ }^{a a^{\prime}}{ }_{c c^{\prime}} c_{a a^{\prime}} \equiv\left\langle\Sigma^{a a^{\prime}} ; c_{b b^{\prime}} \wedge c_{c c^{\prime}}\right\rangle c_{a a^{\prime}} \\
& =-\left[c_{b b^{\prime}}, c_{c c^{\prime}}\right]+\left(\Gamma_{b b^{\prime}} a a^{\prime}{ }_{c c^{\prime}}-\Gamma_{c c^{\prime}} a a^{\prime}{ }_{b b^{\prime}}\right) c_{a a^{\prime}}, \\
& 0=\Delta^{a}{ }_{b c c^{\prime} d d^{\prime}} \equiv\left\langle\Delta^{a}{ }_{b} ; c_{c c^{\prime}} \wedge c_{d d^{\prime}}\right\rangle \\
& =c_{c c^{\prime}}\left(\hat{\Gamma}_{d d^{\prime}}{ }^{a}{ }_{b}\right)-c_{d d^{\prime}}\left(\hat{\Gamma}_{c c^{\prime}}{ }^{a}{ }_{b}\right)-\hat{\Gamma}_{c c^{\prime}}{ }^{f}{ }_{d} \hat{\Gamma}_{f d^{\prime}}{ }^{a}{ }_{b}+\hat{\Gamma}_{d d^{\prime}}{ }^{f}{ }_{c} \hat{\Gamma}_{f c^{\prime}}{ }^{a}{ }_{b}
\end{aligned}
$$

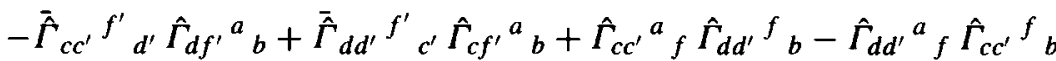

$$
\begin{aligned}
& -\Phi_{c c^{\prime} b d^{\prime}} \varepsilon_{d}^{a}+\Phi_{d d^{\prime} b c^{\prime}} \varepsilon_{c}^{a}+\Omega \phi_{b c d}^{a} \varepsilon_{c^{\prime} d^{\prime}} \\
& 0=\Delta_{c c^{\prime} d d^{\prime}} \equiv\left\langle\Delta ; c_{c c^{\prime}} \wedge c_{d d^{\prime}}\right\rangle \\
& =\hat{\nabla}_{c c^{\prime}} f_{d d^{\prime}}-\hat{\nabla}_{d d^{\prime}} f_{c c^{\prime}}-\Phi_{c c^{\prime} d d^{\prime}}+\Phi_{d d^{\prime} c c^{\prime}}, \\
& 0=\Delta_{a a^{\prime}} b b^{\prime} c c^{\prime} \equiv\left\langle\Delta_{a a^{\prime}}, c_{b b^{\prime}} \wedge c_{c c^{\prime}}\right\rangle \\
& =\hat{\nabla}_{b b^{\prime}} \Phi_{c c^{\prime} a a^{\prime}}-\hat{\nabla}_{c c^{\prime}} \Phi_{b b^{\prime} a a^{\prime}}-K_{a a^{\prime} b b^{\prime} c c^{\prime}}, \\
& 0=\Lambda_{a b c c^{\prime}} \equiv \frac{1}{3}\left\langle\Lambda_{a b}, c_{h c^{\prime}} \wedge c_{c h^{\prime}} \wedge c^{h h^{\prime}}\right\rangle=\nabla_{c^{\prime}} \phi_{a b c h},
\end{aligned}
$$

where $\hat{\nabla}_{a a^{\prime}}, \nabla_{a a^{\prime}}$ denote covariant differentiation with respect to the corresponding connection in the direction of $c_{a a^{\prime}}$. 


\subsection{Conformal geodesics on conformal Einstein spaces}

We shall later employ the conformal geodesic equations to fix a gauge for the conformal Einstein equations. Their use in this context depends on the fact that in Einstein spaces the tensor $\tilde{L}_{\mu \nu}$ entering the conformal geodesic equations has the simple form

$$
\tilde{L}_{\mu \nu} \equiv \frac{1}{n-2}\left(\tilde{R}_{\mu \nu}-\frac{1}{2(n-1)} \tilde{R} \tilde{g}_{\mu \nu}\right)=\bar{\lambda} \tilde{g}_{\mu \nu}, \quad \bar{\lambda}=\frac{1}{2(n-1)} \lambda .
$$

The following two lemmas will allow us to obtain complete information on the quantities which control the conformal gauge. This result is critical for our use of conformal geodesics in the context of the conformal field equations.

Lemma 3.1. Suppose that $x(\tau), b(\tau), c_{k}(\tau)$ is a solution of the conformal geodesics equations (2.49)-(2.51) with respect to the metric $\tilde{g}$ such that $x(\tau)$ is a time-like curve in $\tilde{M}$ defined on some open interval I. If $\tilde{g}$ satisfies the Einstein equations (3.1), then the "conformal factor" $\Theta(\tau)>0$, obtained from (cf. (2.40))

$$
\Theta^{-2} \eta_{k l}=\tilde{g}\left(c_{k}, c_{l}\right)
$$

is given for $\tau \in I$ by

$$
\Theta(\tau)=\Theta_{*}+\left(\tau-\tau_{0}\right) \dot{\Theta}_{*}+\frac{1}{2}\left(\tau-\tau_{0}\right)^{2} \ddot{\Theta}_{*},
$$

where $\tau_{0} \in I, \Theta_{*}=\left.\Theta\right|_{\tau_{0}}>0, \dot{\Theta}_{*}=\left.\dot{\Theta}\right|_{\tau_{0}}, \ddot{\Theta}_{*}=\left.\ddot{\Theta}\right|_{\tau_{0}}$, and the dot denotes the derivative with respect to $\tau$.

We take in (3.41) $k=l$, differentiate, and use (2.51), (3.41) to get

$$
\tilde{\nabla}_{\dot{x}} \Theta=\Theta\langle b, \dot{x}\rangle,
$$

which entails with Eq. (2.49), where we assume $(\dot{x}, \dot{x}) \equiv \tilde{g}(\dot{x}, \dot{x})$,

$$
\tilde{\nabla}_{\dot{x}}\left(\Theta^{2}(\dot{x}, \dot{x})\right)=0 .
$$

Since $(\dot{x}, \dot{x})>0$, we can, possibly after multiplication of $\Theta$ by a constant positive factor, assume that

$$
\Theta^{2}(\dot{x}, \dot{x})=1, \quad \Theta>0, \quad \tau \in l .
$$

By differentiating (3.44) we get

$$
\dot{\Theta}=-\Theta^{3}\left(\dot{x}, \tilde{\nabla}_{\dot{x}} \dot{x}\right) \text {. }
$$

Using (3.42), (3.44) we rewrite Eq. (2.49) as

$$
b=\Theta^{2} \tilde{\nabla}_{\dot{x}} \dot{x}+2 \Theta \dot{\Theta} \dot{x}=\tilde{\nabla}_{\dot{x}}\left(\Theta^{2} \dot{x}\right) .
$$

Inserting this in (2.50), contracting with $\dot{x}$, observing (3.45) and

$$
\left(\tilde{\nabla}_{\dot{x}} \dot{x}, \tilde{\nabla}_{\dot{x}} \dot{x}\right)=\tilde{\nabla}_{\dot{x}}\left(\dot{x}, \tilde{\nabla}_{\dot{x}} \dot{x}\right)-\left(\dot{x}, \tilde{\nabla}_{\dot{x}}^{2} \dot{x}\right)=\frac{1}{2} \tilde{\nabla}_{\dot{x}}^{2}\left(\Theta^{-2}\right)-\left(\dot{x}, \tilde{\nabla}_{\dot{x}}^{2} \dot{x}\right),
$$


we obtain

$$
\left(\dot{x}, \tilde{\nabla}_{\dot{x}}^{2} \dot{x}\right)=\frac{1}{\Theta^{2}}\left\{3\left(\frac{\dot{\Theta}}{\Theta}\right)^{2}-3 \frac{\ddot{\Theta}}{\Theta}+2 \tilde{L}(\dot{x}, \dot{x})\right\} .
$$

Inserting again (3.46) into (2.50) and observing the expressions derived so far yields

$$
\tilde{\nabla}_{\dot{x}}^{2} \dot{x}=-3 \frac{\dot{\Theta}}{\Theta} \tilde{\nabla}_{\dot{x}} \dot{x}+\left\{-3 \frac{\ddot{\Theta}}{\Theta}+\tilde{L}(\dot{x}, \dot{x})\right\} \dot{x}+\frac{1}{\Theta^{2}} \tilde{L}(\dot{x}, \cdot) .
$$

Differentiation of (3.45) gives in view of the equations above

$$
\ddot{\Theta}=\Theta \tilde{L}(\dot{x}, \dot{x})+\frac{1}{2} \Theta^{3}\left(\tilde{\nabla}_{\dot{x}} \dot{x}, \tilde{\nabla}_{\dot{x}} \dot{x}\right)
$$

Taking a further derivative we finally arrive at

$$
\tilde{\nabla}_{\dot{x}}^{3} \Theta=\Theta(\tilde{\nabla} \tilde{L})(\dot{x}, \dot{x}, \dot{x})+3 \dot{\Theta} \tilde{L}(\dot{x}, \dot{x})+3 \Theta \tilde{L}\left(\dot{x}, \tilde{\nabla}_{\dot{x}} \dot{x}\right) .
$$

With (3.40) Eqs. (3.48), (3.50) take the form

$$
\begin{aligned}
& \tilde{\nabla}_{\dot{x}}^{2} \dot{x}=-3 \frac{\dot{\Theta}}{\Theta} \tilde{\nabla}_{\dot{x}} \dot{x}+\left\{-3 \frac{\ddot{\Theta}}{\Theta}+\frac{2 \bar{\lambda}}{\Theta^{2}}\right\} \dot{x}, \\
& \tilde{\nabla}_{\dot{x}}^{3} \Theta=0 .
\end{aligned}
$$

The last equation implies the desired result.

Lemma 3.2. Suppose that $x(\tau), b(\tau), c_{k}(\tau)$ is as in Lemma 3.1 and $\tilde{g}$ satisfies the Einstein equations (3.1). If $\dot{x}=c_{0}$ at $\tau=\tau_{0} \in I$, then

$$
\begin{aligned}
b_{k}(\tau) & =b_{\mu} c_{k}^{\mu}=\Theta^{-1}\left(\dot{\Theta}, d_{a *}\right) \\
& =\Theta^{-1}\left( \pm \sqrt{2 \ddot{\Theta}_{*} \Theta-2 \eta_{00} \bar{\lambda}+\left|d_{c *} d_{*}^{c}\right|}, d_{a *}\right), \quad \tau \in I
\end{aligned}
$$

where $\Theta_{*}^{-1} d_{a *}=\left.b_{a}\right|_{\tau_{0}}$ for $a=1,2,3$.

The field $d_{k}=b_{\mu} e^{\mu}{ }_{k}$ is obtained as solution to Eqs. (2.42), (2.45), (2.46) with $z^{i}=$ $\delta_{0}^{i}$. We can assume, that the orthonormal frame $e_{k}$ with respect to which the equations, in particular the connection coefficients $\tilde{\Gamma}_{i}{ }^{j}{ }_{k}$, are expressed, has been chosen such that $c_{k}=e_{j} C_{k}^{j}=\Theta^{-1} e_{k}$. Then (2.45) reads

$$
\delta^{i}{ }_{k} \dot{\Theta}=\tilde{\Gamma}_{0}{ }^{i}{ }_{k}+S(d)_{0}{ }^{i}{ }_{k}
$$

which gives upon contraction

$$
\dot{\Theta}=d_{0} \text {. }
$$

Eq. (2.46) reads

$$
\dot{d}_{j}=\left(\tilde{\Gamma}_{0}{ }^{k}{ }_{j}+\frac{1}{2} S(d)_{0}{ }^{k}{ }_{j}\right) d_{k} \Theta^{-1}+\bar{\lambda} \eta_{0 j} \Theta^{-1}
$$


These equations imply

$$
\dot{d}_{j}=\frac{1}{2} \eta_{0 j}\left(2 \bar{\lambda}+d_{k} d^{k}\right) \Theta^{-1},
$$

whence

$$
d_{a}(\tau)=d_{a}\left(\tau_{0}\right) \quad \text { for } a=1,2,3,
$$

and

$$
\ddot{\Theta}=\dot{d}_{0}=\frac{1}{2} \eta_{00}\left(2 \bar{\lambda}+d_{k} d^{k}\right) \Theta^{-1}=\frac{1}{2}\left(2 \eta_{00} \bar{\lambda}+\dot{\Theta}^{2}-\left|d_{a} d^{a}\right|\right) \Theta^{-1},
$$

which gives the result.

\section{Remarks.}

(i) We note that Eqs. (3.48), (3.50) constitute a system of differential equations for the projections of conformal geodesic solely in terms of $x(\tau), \Theta(\tau)$. It has been derived without any assumptions on the metric $\tilde{g}$ and therefore holds also if $\tilde{g}$ is a solution to Einstein's field equations with non-zero energy momentum tensor.

(ii) It is an important fact, which will be used in the derivation of the subsidiary equations later on, that the functions $\Theta, d_{k}$ satisfy Eq. (3.55).

(iii) Note that

$$
f_{k}=\Theta^{-1}\left(d_{k}-c_{k}(\Theta)\right)=\Theta^{-1}\left(0, d_{a}-c_{a}(\Theta)\right)
$$

(cf. (3.20)) contains information about the frame and thus is not explicitly known . It may happen that the function $\Theta$ vanishes at some point on a conformal geodesic. If $f_{k}$ and $c_{k}$ remain smooth there, it follows that $d_{a}=c_{a}(\Theta)$ at such points, which allows to calculate the value of $c_{k}(\Theta)$ explicitly. The value of $\eta^{i j} c_{i}(\Theta) c_{i}(\Theta)=$ $\dot{\Theta}^{2}-\delta^{a b} c_{a}(\Theta) c_{b}(\Theta)$ at a point where $\Theta=0$ is by (3.57) the limit of $\dot{\Theta}^{2}-\delta^{a b} d_{a} d_{b}=$ $d^{k} d_{k}=2 \eta_{00} \ddot{\Theta}_{*} \Theta-2 \bar{\lambda}$. It follows that

$$
\eta^{i j} c_{i}(\Theta) c_{j}(\Theta)=-2 \bar{\lambda} \quad \text { where } \Theta=0 .
$$

This behaviour reflects in a most remarkable way the causal properties of conformal boundaries of asymptotically simple space-times.

\section{Anti-de Sitter-type space-times}

\subsection{Anti-de Sitter covering space}

The prototype of a solution to Einstein's equations (3.1) with positive cosmological constant $\lambda$ is given by the universal covering space of the Anti-de Sitter solution (cf. [11]), the Anti-de Sitter covering space (CAdS). Its underlying manifold is $\tilde{M}=\mathbb{R}^{4}$ and the metric is given by

$$
\tilde{g}=(3 / \lambda)\left(\cosh ^{2} r d t^{2}-d r^{2}-\sinh ^{2} r d \sigma^{2}\right),
$$


where $t \in \mathbb{R}, \mathrm{d} \sigma^{2}=\mathrm{d} \theta^{2}+\sin ^{2} \theta \mathrm{d} \phi^{2}$ is the standard line element on the unit 2-sphere, and $r \geq 0, \theta, \phi$ are polar coordinates in $\mathbb{R}^{3}$. In the following we consider the value $\lambda=3$. The space-time is simply connected, geodesically complete, and conformally flat.

The features of this space-time of interest in our analysis are demonstrated particularly clearly by constructing its conformal boundary at space-like and null infinity [11,15-17]. Performing the coordinate transformation

$$
\chi=2 \operatorname{arctg}\left(e^{r}\right)-\frac{1}{2} \pi, \quad 0 \leq \chi<\frac{1}{2} \pi
$$

and rescaling $\tilde{g}$ with the conformal factor $\Omega=1 / \cosh r=\cos \chi$ yields

$$
g=\Omega^{2} \tilde{g}=\mathrm{d} t^{2}-\mathrm{d} \omega^{2}
$$

with $\mathrm{d} \omega^{2}=\mathrm{d} \chi^{2}+\sin ^{2} \chi\left(\mathrm{d} \theta^{2}+\sin ^{2} \theta \mathrm{d} \phi^{2}\right)$, the standard line element on the unit 3-sphere in $\mathbb{R}^{4}$, parametrized by

$$
\begin{aligned}
& x^{1}=\cos \chi, \quad x^{2}=\sin \chi \cos \theta, \quad x^{3}=\sin \chi \sin \theta \cos \phi, \quad x^{4}=\sin \chi \sin \theta \sin \phi, \\
& 0 \leq \chi \leq \pi, \quad 0 \leq \theta \leq \pi, \quad 0 \leq \phi \leq 2 \pi .
\end{aligned}
$$

We have obtained above the embedding of CAdS into the Einstein cosmos, given by the manifold $\mathbb{R} \times S^{3}$ with line element (4.3).

Important for us is the fact that a boundary, the set $\mathcal{I}=\left\{\chi=\frac{1}{2} \pi\right\}$, can be attached to CAdS in such a way that the conformal structure of the metric (4.1), which is represented also by the metric (4.3), extends smoothly through this boundary. Since $\mathcal{I}$ is defined by this property and since it may be shown, that all space-like and null geodesics of CAdS attain two end points on $\mathcal{I}$, it is called the "conformal boundary" of CAdS at space-like and null infinity. We denote by $M$ the manifold with boundary given by the part $0 \leq \chi \leq \frac{1}{2} \pi$ of the Einstein cosmos and denote by $g$ the restriction of the metric (4.3) to $M$.

For completeness we note the well-known fact that time-like geodesics emanating from a given point in CAdS are refocussed such that they keep meeting periodically in an infinite sequence of different points of CAdS $[11,15]$. Taking into account that Anti-de-Sitter space-time admits a symmetry group of maximal dimension, this can be deduced from the following observation. The line element

$$
\mathrm{d} \tau^{2}-\cosh ^{2} \tau\left(\mathrm{d} \eta^{2}+\sinh ^{2} \mathrm{~d} \sigma^{2}\right), \quad|\tau|<\frac{1}{2} \pi, \quad \eta \geq 0,
$$

for which $\tau$ is an affine parameter on time-like geodesics, is transformed by

$$
r=\operatorname{Arsh}(\cos \tau \sinh \eta), \quad t=\operatorname{arctg}(\tan \tau / \cosh \eta)
$$

into the line element (4.1) with $\lambda=3$ and, if we use (4.2) to embed (4.4) into CAdS, the geodesics parametrized by $\tau$ converge at the point $\chi=0, t=-\frac{1}{2} \pi$ as $\tau \rightarrow-\frac{1}{2} \pi$ and at the point $\chi=0, t=\frac{1}{2} \pi$ as $\tau \rightarrow \frac{1}{2} \pi$.

The property, that the conformal boundary is time-like in $(M, g)$, which shows clearly, that CAdS is not globally hyperbolic, will determine the type of PDE problem we shall consider later on. For example, to construct solutions to Maxwells equations on CAdS, data 
have to be prescribed on a space-like hypersurface which extends like $\{t=0\}$ up to $\mathcal{I}$ and on the boundary $\mathcal{I}$ at space-like and null infinity.

We study now a class of conformal geodesics on CAdS. Since these curves are defined by the conformal structure we can analyse them in terms of the metric $g$. The frame field defined by $c_{0}=\partial_{t}$,

$$
\begin{aligned}
c_{1}= & \sin \phi \sin \theta \partial_{\chi}+\left(\frac{\cos \phi}{\sin \theta} \operatorname{ctg} \chi-\sin \phi \operatorname{ctg} \theta\right) \partial_{\phi} \\
& +(\cos \phi+\sin \phi \cos \theta \operatorname{ctg} \chi) \partial_{\theta}, \\
c_{2}= & \cos \phi \sin \theta \partial_{\chi}+\left(-\frac{\sin \phi}{\sin \theta} \operatorname{ctg} \chi-\cos \phi \operatorname{ctg} \theta\right) \partial_{\phi} \\
& +(-\sin \phi+\cos \phi \cos \theta \operatorname{ctg} \chi) \partial_{\theta}, \\
c_{3}= & \cos \theta \partial_{\chi}+\partial_{\phi}-\sin \theta \operatorname{ctg} \chi \partial_{\theta}
\end{aligned}
$$

is orthonormal with respect to the metric $g$ and we have the commutation relations $\left[c_{i}, c_{j}\right]=$ $c_{i}{ }^{l}{ }_{j} c_{l}, c_{i}{ }^{l}{ }_{j} \eta_{l k}=2 \varepsilon_{0 i k j}$ whence $\nabla_{i} c_{j}=\gamma_{i}{ }^{l}{ }_{j} c_{l}, \gamma_{i}{ }^{l}{ }_{j} \eta_{l k}=\varepsilon_{0 i k j}$ and

$$
\begin{aligned}
& R_{j i k l}=\varepsilon_{0}^{m}{ }_{j i} \varepsilon_{0 m k l}, \quad R_{i j}=2\left(\delta^{0}{ }_{i} \delta^{0}{ }_{j}-\eta_{i j}\right), \\
& L_{i j}=\frac{1}{2}\left(R_{i j}-\frac{1}{6} R \eta_{i j}\right)=\delta^{0}{ }_{i} \delta^{0}{ }_{j}-\frac{1}{2} \eta_{i j} .
\end{aligned}
$$

We write $x^{0}=t, x^{1}=\theta, x^{2}=\phi, x^{3}=\chi$. With respect to the frame $c_{j}$ the conformal geodesic equations read

$$
\begin{aligned}
& \dot{x}^{0}=z^{0}, \quad \dot{x}^{\alpha}=z^{a} c_{a}^{\alpha}, \quad \dot{z}^{0}=-2\left(b_{i} z^{i}\right) z^{0}+\left(z_{i} z^{i}\right) b^{0}, \\
& \dot{z}^{a}=-2\left(b_{i} z^{i}\right) z^{a}+\left(z_{i} z^{i}\right) b^{a}, \quad \dot{b}_{0}=\left(b_{i} z^{i}\right) b_{0}-\frac{1}{2}\left(b_{i} b^{i}\right) z_{0}+\frac{1}{2} z_{0}, \\
& \dot{b}_{a}-\varepsilon_{0 c}{ }_{a} z^{c} b_{e}=\left(b_{i} z^{i}\right) b_{a}-\frac{1}{2}\left(b_{i} b^{i}\right) z_{a}-\frac{1}{2} \eta_{a c} z^{c} .
\end{aligned}
$$

The solution of these equations satisfying the initial conditions

$$
x^{\mu}(0)=x_{*}^{\mu}, \quad \dot{x}^{\mu}(0)=\delta^{\mu}{ }_{0}, \quad b_{\mu}=0
$$

is given by

$$
x^{\mu}\left(\tau, x_{*}^{\nu}\right)=x_{*}^{\mu}+2 \delta_{0}^{\mu} \operatorname{arctg}\left(\frac{1}{2} \tau\right), \quad b_{\mu}(\tau)=\frac{1}{2} \tau \delta_{\mu}^{0} .
$$

Various interesting observations follow from this simple result. The first is related to the fact that the "life-time" of conformal geodesics is a somewhat curious notion. If one considers the conformal embedding of Minkowski or de Sitter space-time into the Einstein cosmos (cf. [11]), one finds the following property. In (4.6) the value of $x_{*}^{0}$ can be fixed such that the points $x^{\mu}\left(\tau, x_{*}^{\nu}\right)$, with $\tau \in \mathbb{R}$ and $x_{*}^{a}, a=1,2,3$, parametrizing the points of $S^{3}$, cover the embedded space-times. This property of the global conformal structure of Minkowski and de Sitter space-time stands in sharp contrast to the properties of CAdS. Conformal 
geodesics, considered as curves on the underlying manifold, allow reparametrizations of the form

$$
\tau=\frac{a \bar{\tau}+b}{c \bar{\tau}+d}, \quad a, b, c, d \in \mathbb{R}, \quad a d-b c \neq 0
$$

(cf., e.g. [8]). To cover CAdS by conformal geodesics we would need to apply to (4.6) an infinite number of such reparametrizations, under which the curves (4.6) extend analytically.

Important for our later applications is the fact that the conformal geodesics (4.6) through points of the embedded CAdS stay in the embedded CAdS and that the conformal boundary $\mathcal{I}$ is ruled in a neighbourhood of its intersection with $\{t=0\}$ by conformal geodesics. This allows us to use conformal Gauss coordinates based on the "initial hypersurface" $\{t=0\}$ of CAdS for analysing in a convenient way the space-time and its asymptotic structure "near" $\{t=0\}$.

\subsection{AdS-type space-times}

A smooth orientable and time-orientable four-dimensional space-time $(\tilde{M}, \tilde{g})$ is called "asymptotically simple" $[15,16]$, if there exists a smooth space-time $(M, g)$, a smooth function $\Omega \in C^{\infty}(M)$, and an embedding $j: \tilde{M} \longrightarrow M$, by which we identify $\tilde{M}$ with the open subset $j(\tilde{M})$ of $M$, such that

(i) $\Omega>0$ on $\tilde{M}$, the set $\mathcal{I}=\{\Omega=0\}$ is non-empty and the boundary of $\tilde{M}$ in $M$, and $\mathrm{d} \Omega \neq 0$ on $\mathcal{I}$.

(ii) $g=\Omega^{2} \tilde{g}$ on $\tilde{M}$.

By the first condition $\mathcal{I}$ is a smooth hypersurface in $M$. We will in the following assume that $M$ is a manifold with boundary $\mathcal{I}$ such that $M=\tilde{M} \cup \mathcal{I}$. We shall call $\mathcal{I}$ the "conformal boundary" and $(M, g, \Omega)$ a "conformal extension" of the space-time $(\tilde{M}, \tilde{g})$. The strong smoothness requirements have been made here only for convenience of presentation, but are obviously not necessary for the definition of a conformal boundary. In Section 8 we will refer to the case of lower differentiability. It is customary to add a completeness requirement to the definition of asymptotic simplicity to ensure that nothing is left out in the construction of the conformal boundary. We shall not state such a condition, since in our later consideration it will not be satisfied. However, it will then be clear from the context, how the boundary is to be determined.

In the following will be studied solutions to Einstein field equations (3.1) with cosmological constant $\lambda>0$, which are asymptotically simple. Such solutions will be called "AdS-type space-times". We discuss first a few properties of such spaces.

\subsection{Properties of AdS-type space-times near the conformal boundary}

If $(M, g, \Omega)$ is a conformal extension of an AdS-type space-time, Eq. (3.7) restricted to the conformal boundary implies that $\mathcal{I}$ is a time-like hypersurface because $\lambda>0$. Eq. (3.6), expressed in terms of the conformal Weyl tensor $C^{\mu}{ }_{\nu \lambda \rho}$ of $g$, reads

$$
\Omega \nabla_{\mu} C_{\nu \lambda \rho}^{\mu}-\nabla_{\mu} \Omega C_{\nu \lambda \rho}^{\mu}=0 .
$$


Thus $\nabla_{\mu} \Omega C^{\mu}{ }_{\nu \lambda \rho}=0$ on $\mathcal{I}$ and, since $\nabla_{\mu} \Omega$ is space-like, it follows (most easily in the spinor formalism) that also $C^{\mu}{ }_{\nu \lambda \rho}=0$ on $\mathcal{I}$. Thus we can extend the field $d^{\mu}{ }_{\nu \lambda \rho}$ smoothly to $\mathcal{I}$ and the Einstein equations in the representations (3.3)-(3.7) hold on $M$. These equations are by their definition invariant under the transitions

$$
g \longrightarrow \theta^{2} g, \quad \Omega \longrightarrow \theta \Omega
$$

and associated transformations of the derived fields if $\theta \in C^{\infty}(M), \theta>0$ on $M$. Under such rescalings we have

$$
h \longrightarrow\left(\left.\theta\right|_{\mathcal{I}}\right)^{2} h,\left.\left.\quad \bar{s}\right|_{\mathcal{I}} \longrightarrow\left(\theta^{-1} \bar{s}+\theta^{-2} \nabla^{\mu} \Omega \nabla_{\mu} \theta\right)\right|_{\mathcal{I}},
$$

where $h$ denotes the metric induced by $g$ on $\mathcal{I}$. The constraints (3.8)-(3.17), which hold on $\mathcal{I}$ with $\varepsilon=-1$, simplify considerably, since $\Omega=0$, and $D_{a} \Omega=0$. We assume that the unit normal $n$ of $\mathcal{I}$ points into $\tilde{M}$. After a short calculation we find

$$
\Sigma=c_{3}(\Omega)=\sqrt{\lambda / 3}=\text { const } .>0,
$$

whence $\Sigma$ is invariant under the rescalings (4.7), and

$$
\begin{array}{ll}
\bar{s}=t \sqrt{3 / \lambda}, & \chi_{a b}=t h_{a b}, \quad L_{a}=-D_{a} t, \quad L_{a c}=l_{a b}-\frac{1}{2} t^{2} h_{a c}, \\
D^{a} d_{a c}=0, & d_{a b}^{*}=-\sqrt{(3 / \lambda)} k_{a b},
\end{array}
$$

where

$$
k_{c e} \varepsilon^{e}{ }_{a b}=k_{c a b}=-D_{a} l_{c b}+D_{b} l_{c a}
$$

is the Cotton tensor of $h$ and $t$ is a smooth, gauge-dependent, real-valued function on $\mathcal{I}$. From (4.8) follows that by a suitable choice of scaling (4.7) we can simultaneously and arbitrarily fix the function $t$ and the scaling of $h$. We notice that all fields implied on $\mathcal{I}$ can be expressed in terms of $t$, the inner metric $h$, and tensor fields derived from it.

\subsection{Conformal geodesics near the conformal boundary}

We want to show in the following that conformal geodesics starting on $\mathcal{I}$ with suitable initial conditions stay on the hypersurface $\mathcal{I}$. We keep the assumptions above on the frame $c_{k}$ on $\mathcal{I}$, extend the frame into a neighbourhood $U$ of $\mathcal{I}$ by the requirement $\nabla_{c_{3}} c_{k}=0$ and write again $n=c_{3}$. Then $\Gamma_{3}{ }^{i}{ }_{j}=0$ and $\Gamma_{i j k}=-\Gamma_{i k j}$ on $U$. Furthermore, we assume that $x^{\mu}$ denotes Gauss coordinates on $U$ based on $\mathcal{I}$, such that $\mathcal{I}=\left\{x^{3}=0\right\}$. Then $c^{\mu}{ }_{3}=$ $\delta^{\mu}{ }_{3}, c^{3}{ }_{a}=0$ on $U$.

We express the conformal geodesic equations (2.49), (2.50) on $U$ in terms of $x^{\mu}, b_{k}=$ $b_{\mu} c_{k}^{\mu}$, write $\dot{x}^{\mu}=c_{k}^{\mu} z^{k}$, and find that the equations split into

$$
\begin{aligned}
& \dot{x}^{3}=c^{3}{ }_{k} z^{k}=z^{3}, \quad \dot{z}^{3}=-\Gamma_{a}{ }^{3}{ }_{b} z^{a} z^{b}-2\left(b_{k} z^{k}\right) z^{3}+\left(z_{k} z^{k}\right) b^{3}, \\
& \dot{b}_{3}=\Gamma_{a}{ }^{c}{ }_{3} z^{a} b_{c}+\left(b_{k} z^{k}\right) b_{3}-\frac{1}{2}\left(b_{k} b^{k}\right) z_{3}+L_{33} z^{3}+L_{a 3} z^{a},
\end{aligned}
$$


and, for $a, \alpha=1,2,3$,

$$
\begin{aligned}
& \dot{x}^{\alpha}=c_{a}^{\alpha} z^{a}, \quad \dot{z}^{a}=-\Gamma_{c}{ }^{a}{ }_{b} z^{c} z^{b}-2\left(b_{k} z^{k}\right) z^{a}+\left(z_{k} z^{k}\right) b^{a}, \\
& \dot{b}_{a}=\Gamma_{e}{ }_{a}{ }_{a} z^{e} b_{c}+\left(b_{k} z^{k}\right) b_{a}-\frac{1}{2}\left(b_{k} b^{k}\right) z_{a}+L_{3 a} z^{3}+L_{c a} z^{c} .
\end{aligned}
$$

Since the conformal geodesics depend only on the conformal structure, we may assume a choice of scaling where $t=0$ on $\mathcal{I}$. We give initial data

$$
x^{\mu}(0)=x_{*}^{\mu}, \quad \dot{x}^{\mu}(0)=\dot{x}_{*}^{\mu}, \quad b_{j}(0)=b_{j *}
$$

for a conformal geodesic, such that $x_{*}^{3}=0, \dot{x}_{*}^{3}=0, b_{3 *}=0$, whence also $z^{3}(0)=0$. Thus the solution $x^{\mu}(\tau), z^{k}(\tau), b_{k}(\tau)$ will start with a tangent vector tangent to $\mathcal{I}$ and it follows from the conformal geodesic equations and from the fact that $t=0$ implies $\Gamma_{a}{ }^{3} b=0, \Gamma_{a}{ }^{c} 3=0, L_{3 a}=0$ on $\mathcal{I}$, that the solution is of the form $x^{\mu}(\tau), b_{k}(\tau)$ with $x^{3}(\tau)=0, z^{3}(\tau)=0, b_{3}(\tau)=0$, i.e. the conformal geodesic remains on $\mathcal{I}$. Furthermore, with an ansatz $c=\beta n$ for a solution of Eq. (2.51) along our conformal geodesic, this equation reduces in the present gauge to $\dot{\beta} n=-\langle b, \dot{x}\rangle \beta n$, which clearly has a real-valued solution $\beta \neq 0$. Thus given on some point of our conformal geodesic initial data for a frame which include a vector normal to $\mathcal{I}$, the corresponding solution to (2.51) will be a frame field along the conformal geodesic which contains a vector field normal to $\mathcal{I}$.

Observing now (4.10), we find that $x^{\alpha}(\tau), z^{a}(\tau), b_{a}(\tau)$ satisfy the equations

$$
\begin{aligned}
& \dot{x}^{\alpha}=c^{\alpha}{ }_{a} z^{a}, \quad \dot{z}^{a}=-\Gamma_{c}{ }^{a}{ }_{b} z^{c} z^{b}-2\left(b_{k} z^{k}\right) z^{a}+\left(z_{k} z^{k}\right) b^{a}, \\
& \dot{b}_{a}=\Gamma_{e}{ }^{c}{ }_{a} z^{e} b_{c}+\left(b_{k} z^{k}\right) b_{a}-\frac{1}{2}\left(b_{k} b^{k}\right) z_{a}+l_{c a} z^{c},
\end{aligned}
$$

which are in fact the conformal geodesic equations for the conformal structure which is determined by the interior metric $h$ on $\mathcal{I}$. Furthermore, those of the vector fields solving (2.51) along this conformal geodesic, which are tangent to $\mathcal{I}$, are also obtained as solutions to the interior conformal geodesic equation.

We assume now $x^{\mu}(\tau), z^{k}(\tau), b_{a}(\tau)$ as above for $k, \mu=0,1,2,3, a=0,1,2$. If we perform a rescaling (4.7) which leaves the metric $h$ unchanged and set $b_{3}(\tau)=$ $-\sqrt{(3 / \lambda)} \bar{s}\left(x^{\mu}(\tau)\right)$ with the resulting function $\bar{s}=-\sqrt{(\lambda / 3)} n^{\mu} \nabla_{\mu} \Omega$, we find that this is again a solution to the conformal geodesic equations. We collect these results in the following lemma.

Lemma 4.1. A conformal geodesic in an AdS-type space-time which passes through a point $p$ of the conformal boundary $\mathcal{I}$, is tangent to $\mathcal{I}$ at $p$, and satisfies there $\langle b, n\rangle=$ $-\sqrt{(3 / \lambda)} \bar{s}$, where $n$ is the inward pointing unit normal vector of $\mathcal{I}$ at $p$, remains in $\mathcal{I}$ and defines a conformal geodesic for the interior conformal structure of $\mathcal{I}$. Eqs. (2.51) admits as solutions frame fields on $\mathcal{I}$ which contain a vector field normal to $\mathcal{I}$. Those of the vector fields which are tangent to $\mathcal{I}$ represent solutions of Eq. (2.51) with respect to the interior conformal structure of $\mathcal{I}$. 


\section{Formulation of the initial boundary value problem}

\subsection{General considerations, gauge conditions and initial data}

Suppose that $(M, g, \Omega)$ is a conformal extension of an oriented and time-oriented CAdStype space-time $(\tilde{M}, \tilde{g})$ which does not contain any closed causal curve, but which contains a smooth, oriented, compact, space-like hypersurface $S$ with boundary $\partial S$ which intersects the conformal boundary $\mathcal{I}$ such that $S \cap \mathcal{I}=\partial \mathcal{S}$. We assume that the causal future $J^{+}(S)$ of $S$ coincides with the future domain of dependence of the set $S \cup \mathcal{I}^{+}$and that this set can be mapped diffeomorphically onto $\left[0,1\left[\times S\right.\right.$ such that $\mathcal{I}^{+}$is mapped onto $[0,1[\times \partial S$. We denote here by $\mathcal{I}^{+}$the part of $\mathcal{I}$ in the future of $S$ (including $\partial S$ ). For the causal notions used here and in the following we refer to [11]. The rest of this article is devoted to the initial boundary value problem which we need investigate to answer the question:

Which data implied by $\tilde{g}$ respectively $g, \Omega$ on $S \cup \mathcal{I}^{+}$do we need, to reconstruct in a neighbourhood of $S$ in $J^{+}(S)$ up to diffeomorphisms the solution $\tilde{g}$ of Einstein's field equations (3.1) with positive cosmological constant $\lambda$ ?

It is well known (cf. [4]) that $\tilde{h}, \tilde{\chi}$, the first respectively second fundamental form induced on $\tilde{S}=S \backslash \partial S$ by $\tilde{g}$, are the data which determine the solution $\tilde{g}$ of (3.1) uniquely on the domain of dependence of $\tilde{S}$ up to diffeomorphisms. These fields satisfy the constraint equations

$$
\tilde{D}^{\alpha} \tilde{\chi}_{\alpha \beta}-\tilde{D}_{\beta} \tilde{\chi}_{\alpha}^{\alpha}=0, \quad \tilde{r}-\left(\tilde{\chi}_{\alpha}^{\alpha}\right)^{2}+\tilde{\chi}^{\alpha \beta} \tilde{\chi}_{\alpha \beta}=2 \lambda
$$

induced by (3.1) on $\tilde{S}$, where $\tilde{D}$ denotes the Levi-Civita connection and $\tilde{r}$ the Ricci scalar of $\tilde{h}$. On $\tilde{S}$ we may choose $\Omega=\Omega_{*}$ and $\tilde{\Sigma}=\tilde{n}(\Omega)=\tilde{\Sigma}_{*}$, where $\tilde{n}$ denotes the future directed unit normal field of $\tilde{S}$ with respect to $\tilde{g}$. The functions $\Omega_{*}, \tilde{\Sigma}_{*}$ are restricted only by smoothness and positivity requirements and by their behaviour near $\partial S$, where we require in particular $\Sigma=n(\Omega)=\Omega_{*}^{-1} \tilde{\Sigma}_{*}$ to be smooth. Here $n$ denotes the future directed unit normal field of $S$ with respect to $g$. We can now determine the first respectively second fundamental form

$$
h_{\alpha \beta}=\Omega^{2} \tilde{h}_{\alpha \beta}, \quad \chi_{\alpha \beta}=\Omega\left(\tilde{\chi}_{\alpha \beta}+\Sigma \tilde{h}_{\alpha \beta}\right)
$$

of $g$ on $S$ and, as will be shown later on, all initial data on $S$ for the conformal field equations.

To answer the question, which data may be prescribed on the boundary, we cannot fall back on known results and it should be discussed in conjunction with the evolution equations. To determine the form of these equations we have to decide in particular on a specific gauge, i.e. on a choice of the conformal scaling, of the orthonormal frame, and of a coordinate system near the "initial hypersurface" $\mathcal{I}$. We shall describe now how to fix a gauge in terms of the conformal geodesic equations. To simplify the discussion, it is convenient to assume that the intial hypersurface $S$ has been chosen such that the unit normal vector field $n$ is tangent to $\mathcal{I}$ on $\partial S$, i.e.

$$
\Sigma=n(\Omega)=0 \quad \text { on } \partial S .
$$


This allows us to choose

$$
\Sigma=0 \text { on } S \text {. }
$$

Observing (4.8) the function $\Omega_{*}$ is chosen such that

$$
\bar{s}=0 \quad \text { on } \partial S \text {. }
$$

We assume that at each point of $S$ starts a future directed conformal geodesic $x^{\mu}(\tau), b_{\nu}(\tau)$, $c_{k}(\tau)$ with $\tau=0$ on $S$, such that we have on $\tilde{S}$ in terms of the metric $\tilde{g}$ and its associated Levi-Civita connection $\tilde{\nabla}$

$$
\begin{aligned}
& \dot{x} \quad \text { future directed, orthogonal to } \tilde{S}, \tilde{g}(\dot{x}, \dot{x})=\Omega^{-2}, \\
& b=\Omega^{-1} \mathrm{~d} \Omega \quad \text { whence }\langle b, \dot{x}\rangle=0, \\
& c_{0}=\dot{x}, \quad \tilde{g}\left(c_{i}, c_{j}\right)=\Omega^{-2} \eta_{i j},
\end{aligned}
$$

where the second equation in (5.7) follows from (5.4). We note here that once the gauge has been fixed by (5.6), (5.7), neither the point set run through by a conformal geodesic nor its parameter $\tau$ depends on the remaining freedom of prescribing on $S$ the frame satisfying (5.8).

The following statements will be true in a suitably determined neighbourhood $W$ of $S$ in $J^{+}(S)$ which is chosen such that its intersection with each conformal geodesic is connected. The curves $x^{\mu}(\tau)$ define a smooth, time-like congruence in $W, c_{k}$ a smooth frame field, and $b_{\nu} \mathrm{d} x^{\nu}$ a smooth 1-form. If we set $x^{0}=\tau$ on $W$ and extend a local coordinate system $x^{\alpha}$, $\alpha=1,2,3$, on $S$ into $W$ by the requirement that $x^{\alpha}$ remains constant along the conformal geodesics, we obtain smooth local coordinates $x^{\mu}, \mu=0,1,2,3$, on $W$, which we shall call conformal Gauss coordinates based on $S$. The coefficients $c^{\mu}{ }_{k}=\left\langle\mathrm{d} x^{\mu}, c_{k}\right\rangle$ of $c_{k}$ with respect to the conformal Gauss coordinates satisfy on $W c^{\mu}{ }_{0}=\delta^{\mu}{ }_{0}$, but in general $c^{0}{ }_{a}=0$ only on $S$. We fix a smooth conformal factor $\Omega$ whence the metric $g$ on $W$ by requiring $g\left(c_{i}, c_{k}\right)=\eta_{i k}$. In terms of the metric $g$ and its Levi-Civita connection $\nabla$ the conformal geodesics are represented by $(3.20)$ in the form $x^{\mu}(\tau), f_{\nu}(\tau), c_{k}(\tau)$ with

$$
f_{\mu}=b_{\mu}-\Omega^{-1} \nabla_{\mu} \Omega
$$

such that $f_{\mu}=0$ on $S$. It follows from Lemma 4.1, the assumption (5.5), and the initial conditions that the conformal geodesics starting on $\partial S$ remain in $\mathcal{I}^{+}$. Because of (5.5) we can write $\bar{s}=\Omega_{*} s_{*}$ with a smooth function $s_{*}$ on $S$. From the initial conditions, Lemmas 3.1, 3.2, and (3.17) follows on $W$

$$
\Omega=\Omega_{*}\left(1-\frac{1}{2} \tau^{2} s_{*}\right)
$$

and

$$
b_{k}=\Omega^{-1}\left(\dot{\Omega}, d_{a *}\right) \text { with } d_{a *}=\left(c_{a}^{\alpha} \partial_{\alpha} \Omega\right)_{*},
$$

where we assume, that the functions $\Omega_{*}, s_{*},\left(c^{\alpha}{ }_{a} \partial_{\alpha} \Omega\right)_{*}$, initially defined on $S$, are extended to $W$ such that they are constant along the conformal geodesics. 
We will adopt in the following the notation used in our discussion of the conformal Einstein equations in Section 3. The conformal field equations will be expressed with respect to the connection $\hat{\nabla}$ given by

$$
\hat{\nabla}-\tilde{\nabla}=S(b), \quad \text { respectively } \hat{\nabla}-\nabla=S(f) .
$$

On $S$ the connection $\hat{\nabla}$ coincides with the connection $\nabla$ defined by $g$. Thus we have on $S$ for the connection coefficients with respect to the frame $c_{k}$

$$
\begin{aligned}
& \hat{\Gamma}_{a}{ }^{c}{ }_{b}=\Gamma_{a}{ }^{c}{ }_{b}, \quad-\hat{\Gamma}_{a}{ }^{0}{ }_{c} h^{c b}=\hat{\Gamma}_{a}{ }^{b}{ }_{0}=\chi_{a}{ }^{b}=\chi_{\alpha \beta} c^{\alpha}{ }_{a} c^{\beta}{ }_{c} h^{c b}, \\
& \hat{\Gamma}_{a}{ }^{0}{ }_{0}=0,
\end{aligned}
$$

with

$$
\Gamma_{a}{ }^{d}{ }_{b} c_{d}=D_{c_{a}} c_{b}, \quad \chi_{a b}=g\left(\hat{\nabla}_{c_{a}} c_{0}, c_{b}\right),
$$

where $D$ denotes the Levi-Civita connection defined by the metric $h$ induced by $g$ on $S$. By our choice of gauge we have

$$
\hat{\Gamma}_{0}{ }^{i} k=0, \quad \hat{\Gamma}_{0 j}=0 \quad \text { on } W .
$$

Using (5.9) and the connection defined by $g$ to express (2.35) on $S$, we find

$$
\hat{\Gamma}_{a j}=\left\langle\mathrm{d} f_{j}, c_{a}\right)-L_{j a}=-L_{j a},
$$

where $L_{j k}=A_{\mu \nu} c_{j}^{\mu} c_{k}$ and

$$
A_{\mu \nu}=-\Omega^{-1} \nabla_{\mu} \nabla_{\nu} \Omega+\frac{1}{2} g_{\mu \nu} \Omega^{-2} \nabla_{\sigma} \Omega \nabla^{\sigma} \Omega+\frac{1}{6} \Omega^{-2} \lambda g_{\mu \nu}
$$

since $g$ is conformal to a solution to $\operatorname{Ric}[\tilde{g}]=\lambda \tilde{g}$. Using the data given so far and (5.4), (3.8), (3.16), (3.17) we find

$$
\begin{aligned}
& \hat{\Gamma}_{a 0}=\Omega^{-1} \chi_{a}{ }^{b} D_{b} \Omega, \\
& \hat{\Gamma}_{a b}=\Omega^{-1}\left\{D_{a} D_{b} \Omega-\frac{1}{3} D_{c} D^{c} \Omega h_{a b}\right\}-\frac{1}{12}\left\{r-\left(\chi_{c}{ }^{c}\right)^{2}+\chi_{c d} \chi^{c d}\right\} h_{a b}
\end{aligned}
$$

Using (3.15), (3.16) the rescaled Weyl tensor $d^{i}{ }_{j k l}$ can be determined. It is specified completely by

$$
\begin{aligned}
d_{a b} \equiv d_{a 0 b 0}= & \Omega^{-2}\left\{D_{a} D_{b} \Omega-\frac{1}{3} h_{a b} D^{c} D_{c} \Omega+\Omega\left(l_{a b}-\frac{1}{12} r h_{a b}\right)\right\} \\
& +\Omega^{-1}\left\{\chi^{c}{ }_{c} \chi_{a b}-\chi^{c}{ }_{a} \chi_{c b}-\frac{1}{3} h_{a b}\left(\left(\chi_{c}^{c}\right)^{2}-\chi^{c d} \chi_{c d}\right)\right\}
\end{aligned}
$$

and

$$
d_{a b c} \equiv d_{a 0 b c}=\Omega^{-1}\left\{D_{b} \chi_{c a}-D_{c} \chi_{b a}-h_{a[b} D^{e} \chi_{c] e}+h_{a[b} D_{c]} \chi_{e}^{e}\right\}
$$

or equivalently,

$$
d_{a b}^{*}=-\Omega^{-1} D_{e} \chi_{f(a} \varepsilon_{b}{ }^{e f} .
$$


It can be shown (cf. [6]), that the conformal data so defined satisfy in fact the constraints (3.8)-(3.17) on $\tilde{S}$.

Definition. Initial data $\tilde{h}_{\alpha \beta}, \tilde{\chi}_{\alpha \beta}$ solving on the manifold $\tilde{S}$ the constraint equations implied by (3.1) are said to satisfy the condition of "asymptotic simplicity", if the conformal data derived from them as above extend smoothly to $\partial S$.

This definition raises the question of "how many" such data exist. This problem will not be discussed in detail in this article. However, to illustrate the nature of the problem, we remark on an important subclass of data for which the complete analysis has been given.

In analogy to the condition of time-symmmetry in the standard Cauchy problem for Einstein's field equations we consider the case

$$
\tilde{\chi}_{a b}=0 \text { on } \tilde{S} .
$$

It follows then from (5.4) that also $\chi_{a b}=0$ on $S$. As described above, we can then determine all conformal data on $S$ from $\tilde{h}_{a b}$. We find from (3.9), (3.15), (3.16), (3.8) that $L_{a}=0$, $d_{a b c}=0, \frac{1}{4} r=l_{a}^{a}=L_{a}^{a}, \frac{1}{3} D_{a} D^{a} \Omega=-\Omega \frac{1}{12} r+\bar{s}$ on $S$ and thus can write (3.17) in the form

$$
2 \Omega D_{a} D^{a} \Omega=-\frac{1}{2} r \Omega^{2}+\lambda+3 D_{a} \Omega D^{a} \Omega .
$$

Setting in this equation $\Omega=1$, or rewriting it in terms of the metric $\tilde{h}=\Omega^{-2} h$ induced by $\tilde{g}$ on $S \backslash \partial S$, we see that (5.18) is equivalent to the Hamiltonian constraint $\tilde{r}=2 \lambda=$ const. $>0$ which we assumed to be satisfied by $\tilde{h}_{a b}$.

The construction of solutions to this constraint, which satisfy the fall-off conditions which are implicit in the smoothness requirement on the conformal fields, has been described in all details in [2] where the following result has been shown (observe the different signature used in that article, the metric $h$ used here is negative definite).

Suppose $\lambda$ is a positive real number, $S$ is a smooth, compact, orientable, three-dimensional manifold with boundary $\partial S$, and $h$ is a smooth, negative definite metric field on $S$. Denote by $\mu$ the second fundamental form induced by $h$ on $\partial S$ and by $\mu^{*}$ its trace free part with respect to the first fundamental form on $\partial S$. If and only if the metric $h$ is such that the conformally invariant condition

$$
\mu^{*}=0 \text { on } \partial S
$$

is satisfied, there exists a (unique) solution $\Omega \in C^{\infty}(S)$ of (5.18) with $\Omega>0$ on $S \backslash \partial S$, $\Omega=0, \mathrm{~d} \Omega \neq 0$ on $\partial S$ and it follows that the fields given on $S \backslash \partial S$ by

$$
\begin{aligned}
& \Sigma=0, \quad \bar{s}=\frac{1}{3} D_{a} D^{a} \Omega+\frac{1}{12} \Omega r, \quad L_{a}=0, \quad d_{a b c}=0, \\
& L_{a b}=-\Omega^{-1}\left(D_{a} D_{b} \Omega-\frac{1}{3} D_{c} D^{c} \Omega h_{a b}\right)+\frac{1}{12} r h_{a b}, \\
& d_{a b}=\Omega^{-2}\left\{D_{a} D_{b} \Omega-\frac{1}{3} D_{c} D^{c} \Omega+\Omega\left(l_{a b}-\frac{1}{12} r h_{a b}\right)\right\}
\end{aligned}
$$

extend smoothly to $S$ and solve there the constraint equations (3.8)-(3.17). 
It turns out that if the condition (5.19) is not satisfied, there still exists a unique solution $\Omega$ of (5.18) which is positive on $S \backslash \partial S$ and vanishes on $\partial S$. This solution will be smooth on $S$ also under conditions slightly weaker than (5.19). In any case, if (5.19) is not satisfied, the field $d_{a b}(p)$ diverges like $\rho(p)^{-2}$ as the distance $\rho(p)$ of the point $p \in \tilde{S}$ from the part of the boundary $\partial S$ where (5.19) is violated goes to zero. Most likely this blow-up behaviour will spread and the solution of the conformal field equations determined by the data on $S \backslash \partial S$ will blow up at parts of the boundary of its domain of existence. If such a spreading of the blow-up behaviour does occur, it should "most easily" be established by using the conformal field equations, which would allow to localize the problem to a neighbourhood of $\partial S$. Since the conformal factor will be bounded below by a positive number near most parts of the boundary of the domain of existence, also the "physical" conformal Weyl tensor will diverge at parts of the boundary. Thus we would obtain a statement on the blow-up behaviour of the physical fields not depending on the use of the conformal method. This suggests that smooth AdS-type space-times with boundary at space-like and null infinity which is diffeomorphic to $\mathbb{R} \times \partial S$ exist only if the initial data implied by the "physical" metric $\tilde{g}$ satisfy the fall-off conditions encoded in the smoothness requirement of asymptotic simplicity.

Recently, in a context somewhat different from the one discussed here, the investigations in [2] have been generalized into various directions, in particular to the case where the second fundamental form $\tilde{\chi}_{a b}$ does not vanish [1]. By the methods developed in [1] the existence of a more general class of data satisfying the condition of asymptotic simplicity can be shown.

\subsection{The reduced field equations}

In the following $c_{k}$ will always denote the specific frame field on $\tilde{M}$ determined by the gauge discussed in Section 5.1 and $\sigma^{k}$ the dual 1 -form field. We shall employ a splitting of fields with respect to the time-like vector field $\sqrt{2} c_{0}$, which in the frame $c_{a a^{\prime}}$ has the representation

$$
\tau^{a a^{\prime}}=\varepsilon_{0}{ }^{a} \varepsilon_{0^{\prime}}^{a^{\prime}}+\varepsilon_{1}^{a} \varepsilon_{1^{\prime}}^{a^{\prime}} .
$$

With the exception of the factor $\sqrt{2}$ in (5.21) we will follow the conventions of [7]. For later use we need, however, more details about the underlying structures. The coefficients (5.20) are used to define the projected van der Waerden symbols

$$
\sigma_{j}^{a b}=\sigma_{j}^{(a}{ }_{a^{\prime}} \tau^{b) a^{\prime}}, \quad \sigma_{a b}^{j}=\tau_{(b}{ }^{a^{\prime}} \sigma_{a) a^{\prime}},
$$

such that $\sigma_{0}{ }^{a b}, \sigma^{0}{ }_{a b}$ both vanish and

$$
\begin{aligned}
& \delta_{k}^{j}=\sigma_{a b}^{j} \sigma_{k}^{a b}, \quad j=1,2,3, \quad \varepsilon_{a}^{b} \varepsilon_{a^{\prime}}^{b^{\prime}}=\frac{1}{2} \tau_{a a^{\prime}} \tau^{b b^{\prime}}+\sigma^{j}{ }_{a f} \tau^{f}{ }_{a^{\prime}} \tau^{e b^{\prime}} \sigma_{j e}{ }^{b}, \\
& \eta_{j k} \sigma_{a b}^{j} \sigma_{c d}^{k}=-\delta_{j k} \sigma_{a b}^{j} \sigma_{c d}^{k}=-\varepsilon_{a(c} \varepsilon_{d) b} \equiv h_{a b c d} \text {. }
\end{aligned}
$$

The simply connected group which leaves $\varepsilon_{a b}$ as well as $\tau_{a b^{\prime}}$ invariant is given by

$$
S U(2, C)=\left\{t^{a}{ }_{b} \in S L(2, C) \mid \tau_{a a^{\prime}} t^{a}{ }_{b} \bar{t}^{a^{\prime}}{ }_{b^{\prime}}=\tau_{b b^{\prime}}\right\} .
$$


There is a surjective $2: 1$ group homomorphism

$$
S U(2, C) \ni t_{b}^{a} \stackrel{\Phi}{\prime \prime}^{i} t_{j}=\sigma_{a b}^{i} t_{c}^{a} t_{d}^{b} \sigma_{j}{ }^{c d} \in O_{+}(3, \mathbb{R}),
$$

where $i, j=1,2,3$. It implies an isomorphism of Lie algebras which is given by

$$
s u(2, C) \ni u_{b}^{a} \stackrel{\Phi_{*}^{\prime \prime}}{\rightarrow} u_{j}^{i}=2 \sigma_{a b}^{i} u_{c}^{a} \sigma_{j}{ }^{c b} \in o(3, \mathbb{R})
$$

and has inverse

$$
o(3, \mathbb{R}) \ni u_{j}^{i} \stackrel{\Phi_{*}^{\prime \prime}-1}{\longrightarrow} u_{b}^{a}=\frac{1}{2} u_{j}^{i} \sigma_{i}^{a c} \sigma_{b c}^{j} \in \operatorname{su}(2, C) .
$$

For any frame $e_{a a^{\prime}}=e_{k} \sigma_{a a^{\prime}}^{k}$ with $e_{0}=c_{0}$ we get a decomposition

$$
e_{a a^{\prime}}=\frac{1}{2} \tau_{a a^{\prime}} \tau^{b b^{\prime}} e_{b b^{\prime}}-\tau_{a^{\prime}}^{b} e_{a b}
$$

with $\tau^{b b^{\prime}} e_{b b^{\prime}}=\sqrt{2} c_{0}$ and a frame $e_{a b}=\tau_{(b} a^{\prime} e_{a) a^{\prime}}$ in the subspace orthogonal to $c_{0}$ which satisfies, with respect to the metric $h$ induced by $g$ on that subspace, the normalization condition $h\left(e_{a b}, e_{c d}\right)=h_{a b c d}$.

At $p \in \tilde{M}$ the vector $c_{0}$ induces a hermitian form which for spinors $\kappa, \mu$ is defined by $(\kappa, \mu) \rightarrow \sqrt{2} g\left(c_{0}, \kappa \bar{\mu}\right)$. The set of spin frames $\delta \in S L(\tilde{M})$ which are normalized with respect to this hermitian form by $\sqrt{2} g\left(c_{0}, \delta_{a} \bar{\delta}_{a^{\prime}}\right)=\tau_{a a^{\prime}}$ defines a subbundle $S U(\tilde{M})$ of $S L(\tilde{M})$ with structure group $S U(2, C)$. The structure of this group is reflected by the property of the spin frames $\delta \in S U(\tilde{M})$ that the normalized spinor $\delta_{0}$ determines uniquely the spinor $\delta_{1}$ and vice versa.

The map which associates with $\delta \in S U(\tilde{M})$ the frame $e_{a b} \sigma_{k}^{a b}$ is a $2: 1$ bundle morphism of $S U(\tilde{M})$ onto the bundle of positively oriented frames orthogonal to $c_{0}$ with respect to the homomorphism $\Phi^{\prime \prime}$.

Any spinor field on $\tilde{M}$ supplies an invariant function on $S U(\tilde{M})$ and vice versa. If $\mu_{a^{\prime}}$ is the function corresponding to a primed spinor field, we define a bijective complex linear map $\mu_{a^{\prime}} \rightarrow \mu_{a}=\tau_{a} a^{\prime} \mu_{a^{\prime}}$ which allows to associate with any primed spinor field an unprimed spinor field. This map is extended in an obvious way to spinor fields of higher valence and various index positions such that it commutes with contractions. It should be observed that the position of the contracted index of $\tau^{a a^{\prime}}$ is always chosen as shown above. The map is related to the hermitian conjugation map which associates to any unprimed spinor field another such field by $\mu_{a} \rightarrow \mu_{a}^{+}=\tau_{a}{ }^{\prime} \bar{\mu}_{a^{\prime}}$. Again, this map is extended by complex semi-linearity to more general spinor fields such that it commutes with contractions and satisfies, e.g. $\mu_{a_{1} \ldots a_{k}}^{++}=(-1)^{k} \mu_{a_{1} \ldots a_{k}}$.

If a tensor field $\mu$ is represented at $\delta \in S U(\tilde{M})$ by the components $\mu_{a_{1} a_{1}^{\prime} \ldots a_{k} a_{k}^{\prime}}$, it can be expanded in terms of the components of the frame $e_{a b}=\delta_{(a} \tau_{b)} a^{\prime} \bar{\delta}_{a^{\prime}}$ orthogonal to $c_{0}$ if and only if it is "spatial", i.e. if $\tau^{a_{j} a_{j}^{\prime}} \mu_{a_{1} a_{1}^{\prime} \ldots a_{j} a_{j}^{\prime} \ldots a_{k} a_{k}^{\prime}}=0$ for $j=1, \ldots, k$. In that case the components of $\mu$ in the frame $e_{a b}$ are given by

$$
\mu_{a_{1} b_{1} \ldots a_{k} b_{k}}=\tau_{b_{1}} a_{1}^{\prime} \ldots \tau_{b_{k}} a_{k}^{\prime} \mu_{a_{1} a_{1}^{\prime} \ldots a_{j} a_{j}^{\prime} \ldots a_{k} a_{k}^{\prime}}=\mu_{\left(a_{1} b_{1}\right) \ldots\left(a_{k} b_{k}\right)}
$$


Conversely, any spinor field with $2 k$ unprimed indices which has the symmetries in the index pairs considered above corresponds to a spatial spinor. For a tensor field $\mu$ which is represented on $S U(\tilde{M})$ by a component function $\mu_{a a^{\prime}}$ this tells us, that we obtain an orthogonal splitting by writing $\mu_{a a^{\prime}}=\tau_{c a^{\prime}} \tau^{c b^{\prime}} \mu_{a b^{\prime}}=\frac{1}{2} \tau_{a a^{\prime}} \tau^{c c^{\prime}} \mu_{c c^{\prime}}-\tau_{a^{\prime}} \mu_{c a}$, where $\mu_{c a}=$ $\tau_{(c}{ }^{\prime} \mu_{a) b^{\prime}}$ represents the spatial part of $\mu$. Again, this procedure can be extended in an obvious way to spinors of higher valence.

Finally we note that a spatial tensor which is represented by the component function $\mu_{a_{1} b_{1} \ldots a_{k} b_{k}}=\mu_{\left(a_{1} b_{1}\right) \ldots\left(a_{k} b_{k}\right)}$ represents a real tensor field if and only if it satisfies the reality condition $\mu_{a_{1} b_{1} \ldots a_{k} b_{k}}^{+}=(-1)^{k} \mu_{a_{1} b_{1} \ldots a_{k} b_{k}}$.

We shall discuss now the data and the propagation equations inherent in the conformal Einstein equations in terms of spinors and the gauge discussed in Section 5.1. By $x^{\mu}$ will be denoted conformal Gauss coordinates and we will sometimes write as before $\tau=x^{0}$.

The frame vectors are projected onto

$$
\tau^{a a^{\prime}} c_{a a^{\prime}}=\sqrt{2} \partial_{\tau}, \quad c_{a b}=\tau_{(a}^{a^{\prime}} c_{b) a^{\prime}}=c_{a b}^{\mu} \partial_{\mu} .
$$

We set

$$
\begin{aligned}
& \Gamma_{a b c d}=\tau_{b}{ }^{b^{\prime}} \Gamma_{a b^{\prime} c d}, \quad f_{a b}=\tau_{b}{ }^{b^{\prime}} f_{a b^{\prime}}, \\
& \sqrt{2} \chi_{a b c d} \equiv-\Gamma_{a b d c}+\tau_{b}{ }^{b^{\prime}} \tau_{c}{ }^{c^{\prime}} \tau_{d}{ }^{d^{\prime}} \bar{\Gamma}_{a b^{\prime} c^{\prime} d^{\prime}}=\tau_{b}{ }^{a^{\prime}}\left(\nabla_{a a^{\prime}} \tau_{c c^{\prime}}\right) \tau_{d}{ }^{c^{\prime}}, \\
& \Phi_{a c b d}=\tau_{c}{ }^{a^{\prime}} \tau_{d}{ }^{b^{\prime}} \Phi_{a a^{\prime} b b^{\prime}} .
\end{aligned}
$$

From the gauge conditions (5.13) follow

$$
\begin{aligned}
& f_{a b}=f_{b a}, \quad \Gamma_{c}{ }^{c}{ }_{a b}=\tau^{c c^{\prime}} \Gamma_{c c^{\prime} a b}=-f_{a b}, \\
& \sqrt{2} \chi_{(a b) c d}=\sqrt{2} \chi_{a b c d}-\varepsilon_{a b} f_{c d}, \quad \Phi_{c}{ }^{c} b d=0 .
\end{aligned}
$$

The initial data on $\tilde{S}$ are given in terms of spinor expressions as follows:

(i) The coefficients $c^{\alpha}{ }_{a b}$ are determined by the choice of the frame $c_{k}$. This choice is not critical away from the boundary $\partial S$. The specific choice near the boundary will be discussed later on. By our gauge conditions $c^{0}{ }_{a b}=0$.

(ii) Again, by our gauge $f_{a b}=0$.

(iii) Let $D_{a b}$ denote the covariant derivative in the direction of $c_{a b}$ with respect to the connection $D$ and define connection coefficients $\gamma_{a b c d e f}$ by setting $D_{a b} c_{e f}=\gamma_{a b}{ }^{c d}{ }_{e f} c_{c d}$. The coefficients $\gamma_{a b c d}=-\frac{1}{2} \gamma_{a b c f d}{ }^{f}$ satisfy $\gamma_{a b c d}=\gamma_{(a b)(c d)}$. The second fundamental form on $S$ is given by $\chi_{a b c d}=g\left(\nabla_{c_{a b}} c_{0}, c_{c d}\right)$ and coincides because of our gauge conditions with the restriction of $(5.21)$ to $S$. It satisfies $\chi_{a b c d}=\chi_{(a b)(c d)}=\chi_{c d a b}$. The connection coefficients $\Gamma_{a b c d}$ are given by

$$
\Gamma_{a b c d}=\gamma_{a b c d}-\frac{1}{\sqrt{2}} \chi_{a b c d} .
$$

Defining for the function $\gamma_{a b c d}$ the operation of hermitian conjugation by the same formal rule as for invariant functions, we find that the two terms in the last expression may be distinguished by using the properties

$$
\gamma_{a b c d}^{+}=-\gamma_{a b c d}, \quad \chi_{a b c d}^{+}=\chi_{a b c d} .
$$


(iv) It follows directly from (5.13), (5.14) that

$$
\begin{aligned}
\Phi_{a b c d}= & \Omega^{-1} D_{a b} D_{c d} \Omega-\frac{1}{6} \Omega^{-2} h_{a b c d}\left\{3 D_{e f} \Omega D^{e f} \Omega+\lambda\right\} \\
& -\varepsilon_{c d} \frac{1}{\sqrt{2}} \Omega^{-1} \chi_{a b}{ }^{e f} D_{e f} \Omega \\
= & \Omega^{-1} D_{(a b} D_{c d)} \Omega-\frac{1}{12}\left\{r-\left(\chi_{e f} e f\right)^{2}+\chi_{e f g h} \chi^{e f g h}\right\} h_{a b c d} \\
& -\varepsilon_{c d} \frac{1}{\sqrt{2}} \Omega^{-1} \chi_{a b}{ }^{e f} D_{e f} \Omega .
\end{aligned}
$$

(v) Denoting by $s_{a b c d}$ the trace-free part of the Ricci tensor of $h$, we finally get

$$
\begin{aligned}
\phi_{a b c d}= & d_{a b c d}+i d_{a b c d}^{*}=\Omega^{-2} D_{(a b} D_{c d)} \Omega \\
& +\Omega^{-1}\left(S_{a b c d}+\chi^{e f}{ }_{e f} \chi_{(a b c d)}-\chi^{e f}{ }_{(a b} \chi_{c d) e f}+\sqrt{2} D^{f}{ }_{(a} \chi_{b c d) f}\right) .
\end{aligned}
$$

It may be noticed that the first four terms on the right-hand side of the last equation represent real spatial tensors while the last term represents a purely imaginary spatial tensor on $\tilde{S}$. This corresponds to the decomposition into the electric part $d_{a b c d}$ and the magnetic part $d_{a b c d}^{*}$ which both represent real spatial tensors.

The propagation equation will be expressed with respect to the covariant directional derivative operators

$$
P=\tau^{a a^{\prime}} \nabla_{a a^{\prime}}, \quad \mathcal{D}_{a b}=\tau_{(a}^{a^{\prime}} \nabla_{b) a^{\prime}},
$$

given explicitly by

$$
\begin{aligned}
& P=\nabla_{00^{\prime}}+\nabla_{11^{\prime}}=\sqrt{2} \partial_{\tau}, \quad \mathcal{D}_{01}=\frac{1}{2}\left(\nabla_{00^{\prime}}-\nabla_{11^{\prime}}\right), \\
& \mathcal{D}_{00}=-\nabla_{01^{\prime}}, \quad \mathcal{D}_{11}=\nabla_{10^{\prime}} .
\end{aligned}
$$

We want to extract from Eqs. (3.35) to (3.39) a system of propagation equations for the unknowns

$$
c_{a b}^{\mu}, \Gamma_{a b c d}, \Phi_{a c b d}, \phi_{a c b d} .
$$

These equations will imply in particular equations for

$f_{a b}, \quad \chi_{a b c d}$,

which for later convenience will be added to the list. For Eqs. (3.35)-(3.38) the choice of evolution equations is suggested by our gauge conditions. Contracting these equations with $\tau^{c c^{\prime}}$ and projecting as indicated above, we get with $\Omega$ as in (5.10) and with

$$
d_{a b}=\left(c^{\alpha}{ }_{a b} \partial_{\alpha} \Omega\right)_{*},
$$

derived from (5.11),

$$
\begin{aligned}
& \partial_{\tau} c_{a b}^{0}=-f_{a b}-\chi_{(a b)}{ }^{e f} c^{0}{ }_{e f}, \\
& \partial_{\tau} c_{a b}^{\alpha}=-\chi_{(a b)}{ }^{e f} c^{\alpha}{ }_{e f}, \quad \alpha=1,2,3, \\
& \partial_{\tau} f_{a b}=-\chi_{(a b)}{ }^{e f} f_{e f}-\frac{1}{\sqrt{2}} \Phi_{a b f} f
\end{aligned}
$$




$$
\begin{aligned}
\partial_{\tau} \chi_{a b c d}= & -\chi_{(a b)}{ }^{e f} \chi_{e f c d}-\frac{1}{\sqrt{2}} \varepsilon_{a b} \chi_{(c d)}{ }^{e f} f_{e f}+\Phi_{a b(c d)} \\
& -\frac{1}{2} \varepsilon_{a b} \Phi_{c d f} f-\frac{1}{2} \Omega\left(\phi_{a b c d}+\phi_{a b c d}^{+}\right), \\
\partial_{\tau} \Gamma_{a c b d}= & -\chi_{(a c)}{ }^{e f} \Gamma_{e f b d}+\frac{1}{2}\left(\varepsilon_{a b} \chi_{(c d) e f}+\varepsilon_{a d} \chi_{(b c) e f}\right) f^{e f}-\chi_{(a c)(b}{ }^{e} f_{d) e} \\
& -\frac{1}{\sqrt{2}}\left(\Phi_{a c d b}-\varepsilon_{a b} \Phi_{c d f} f\right)+\frac{1}{\sqrt{2}} \Omega \phi_{a b c d}, \\
\partial_{\tau} \Phi_{a c b d}= & -\chi_{(a c)}{ }^{e f} \Phi_{e f b d}-\frac{1}{2} \partial_{\tau} \Omega\left(\phi_{a b c d}+\phi^{+}{ }_{a b c d}\right) \\
+ & \frac{1}{\sqrt{2}}\left(d^{e}{ }_{d} \phi_{a b c e}-d^{e}{ }_{b} \phi^{+}{ }_{a c d e}\right) .
\end{aligned}
$$

We consider two ways to deduce useful evolution equations from (3.39). We have the splitting

$$
\Lambda_{a b c d}=\tau_{d} d^{\prime} \Lambda_{a b c d^{\prime}}=\tau_{d}^{d^{\prime}} \nabla_{d^{\prime}}^{f} \phi_{a b c f}=P_{a b c d}-\frac{3}{4} \varepsilon_{d(c} C_{a b)}
$$

with

$$
P_{a b c d}=\Lambda_{(a b c d)}=-\frac{1}{2}\left(P \phi_{a b c d}-2 \mathcal{D}_{(d} f_{\phi_{a b c) f}}\right), \quad C_{a b}=\Lambda_{a b f}{ }^{f}=\mathcal{D}^{e f} \phi_{a b e f}
$$

The equations

$$
0=-2 P_{a b c d}=P \phi_{a b c d}-2 \mathcal{D}_{(d} f_{\phi_{a b c) f}}
$$

can be used as propagation equations for the rescaled Weyl spinor $\phi_{a b c d}$ (cf. [7]). If we introduce the unknown

$$
\psi_{a b c d} \equiv \kappa_{a b c d} \phi_{a b c d}
$$

obtained by multiplying the components of the spinor field with the square root of suitable binomial coefficients

$$
\kappa_{a b c d} \equiv \sqrt{\left(\begin{array}{c}
4 \\
a+b+c+d
\end{array}\right)},
$$

the propagation equations can be rewritten as an equation of the form

$$
\partial_{\tau} \psi+A^{a b} c_{a b}^{\mu} \partial_{\mu} \psi=B(\Gamma, \psi)
$$

for the unknown "column vector" $\psi$ with complex-valued components

$$
\psi_{p} \equiv \psi_{a b c d}, \quad p=a+b+c+d=0,1,2,3,4
$$

The right member is a linear function of the $\psi_{p}$ with coefficients given by the $\Gamma_{a b c d}$. The constant matrices $A^{a b}$ are such that the system is symmetric hyperbolic. We shall call the system consisting of Eqs. (5.24)-(5.29) and (5.30), or equivalently (5.31), the "standard system" of reduced field equations. We note for later comparison, that for (5.31) we have 


$$
A^{01}=A^{10}=\frac{1}{\sqrt{2}}\left(\begin{array}{ccccc}
-1 & 0 & 0 & 0 & 0 \\
0 & -\frac{1}{2} & 0 & 0 & 0 \\
0 & 0 & 0 & 0 & 0 \\
0 & 0 & 0 & \frac{1}{2} & 0 \\
0 & 0 & 0 & 0 & 1
\end{array}\right) \text {. }
$$

The standard system is well suited to study the evolution of the fields in regions away from the boundary $\mathcal{I}$ and it has the advantage of being given by a simple analytic expression. However, it turns out that we can extract from (3.39) another system of symmetric hyperbolic propagation equations which simplifies the analysis of the evolution near a time-like boundary considerably. This system is given by

$$
\begin{aligned}
& -2 P_{0000}=0, \quad-2 \sqrt{2} P_{0001}-\frac{1}{\sqrt{2}} C_{00}=0, \quad-2 \sqrt{2} P_{0011}=0, \\
& -2 \sqrt{2} P_{0111}+\frac{1}{\sqrt{2}} C_{11}=0, \quad-2 P_{1111}=0 .
\end{aligned}
$$

Expressed in terms of the unknowns

$$
\begin{aligned}
& \psi_{p} \equiv c_{p} \phi_{a b c d}, \quad p=a+b+c+d \\
& c_{0}=c_{4}=1, \quad c_{1}=c_{2}=c_{3}=\sqrt{2}
\end{aligned}
$$

it also takes the form (5.31). We have in this case

$$
\begin{aligned}
A^{01} & =A^{10}=\left(\begin{array}{ccccc}
-\frac{1}{\sqrt{2}} & 0 & 0 & 0 & 0 \\
0 & 0 & 0 & 0 & 0 \\
0 & 0 & 0 & 0 & 0 \\
0 & 0 & 0 & 0 & 0 \\
0 & 0 & 0 & 0 & \frac{1}{\sqrt{2}}
\end{array}\right), \\
A^{00} & =\left(\begin{array}{ccccc}
0 & \sqrt{2} & 0 & 0 & 0 \\
0 & 0 & 1 & 0 & 0 \\
0 & 0 & 0 & 1 & 0 \\
0 & 0 & 0 & 0 & \sqrt{2} \\
0 & 0 & 0 & 0 & 0
\end{array}\right)=-{ }^{T} A^{11} .
\end{aligned}
$$

The form of the matrix $A^{01}$ is decisive for the discussion of the boundary value problem. We shall call the system consisting of Eqs. (5.24)-(5.29) and (5.33) the "boundary adapted system" of reduced field equations. The system is symmetric hyperbolic.

Remark. As compared with the conformal representation of the Einstein equations used in [7] and previous articles, the new conformal representation shows two important advantages. On the one hand we have complete information on the conformal factor in terms of the initial data. Thus the "location of infinity" is known a priori in terms of our chosen coordinates. On the other hand the analysis of the reduced field equations is considerably simplified by the fact that Eqs. (5.24)-(5.29) are ordinary differential equations, if the field $\phi_{a b c d}$ is considered as given. Thus the question, which boundary data may be prescribed in the initial 
boundary value problem considered here, is essentially reduced to an investigation of the properties of the system (3.39). Both facts become even more important in the analysis of the asymptotic behaviour of the solutions of Einstein's field equations with cosmological constant $\lambda=0$.

\subsection{The structure of the normal matrix and the boundary conditions}

To study the solution of the conformal field equations near the boundary $\mathcal{I}$, we have to choose coordinates and frame vector fields adapted to the boundary. The following discussion will refer to a suitable open neighbourhood $U$ in $W$ of a given point on $\partial S$ which is determined such that in particular the intersections of $U$ with conformal geodesics are connected and that

$$
1+\frac{1}{2} \tau^{2} s_{*}>0 \text { on } U
$$

We assume that the frame $c_{a}$ on $S \cap U$ is chosen such that $c_{3}$ is orthogonal to and inward directed at $\partial S$ and $D_{c_{3}} c_{a}=0$ on $S \cap U$, where $D$ denotes the inner covariant derivative on $S$. Furthermore, we choose coordinates $x^{\alpha}, \alpha=1,2,3$ on $S \cap U$ such that $x^{3}$ vanishes on $\partial S$ and $\left\langle\mathrm{d} x^{\alpha}, c_{3}\right\rangle=\delta^{\alpha}{ }_{3}$ on $S \cap U$. A conformal Gauss gauge satisfying such assumptions near $\partial S$ will be called "boundary-adapted gauge". We set

$$
\rho^{a a^{\prime}}=\varepsilon_{0}{ }^{a} \varepsilon_{0^{\prime}}^{a^{\prime}}-\varepsilon_{1}^{a} \varepsilon_{1^{\prime}}^{a^{\prime}}, \quad \rho_{a b}=\tau_{b}^{a^{\prime}} \rho_{a a^{\prime}}=-2 \varepsilon_{(a}{ }^{0} \varepsilon_{b)}{ }^{1} .
$$

The constant coefficients in the relation $c_{a a^{\prime}}=c_{k} \sigma^{k}{ }_{a a^{\prime}}$ are chosen as usual such that we have real vectors $\sqrt{2} c_{0}=\tau^{a a^{\prime}} c_{a a^{\prime}}, \quad \sqrt{2} c_{3}=\rho^{a a^{\prime}} c_{a a^{\prime}}=\rho^{a b} c_{a b}=2 c_{01}$ on $S \cap U$. With this choice of frame (3.17) entails

$$
c_{a b}(\Omega)=d_{a b}=-\sqrt{(\lambda / 6)} \rho_{a b} \quad \text { on } \partial S .
$$

We write $\hat{\chi}_{a b c d}=\chi(a b) c d$ and split all fields on $\mathcal{I}$ into their parts orthogonal and tangent to $\mathcal{I}$. Contraction with $\rho^{a b}$ will be denoted by an upper index $\perp$ and projection onto the part tangent to $\mathcal{I}$ by an upper index $\mid$. These indices will appear on the left respectively on the right, if the operation is performed on the first respectively on the second pair of indices of the fields considered in the following. From (5.36), (3.9), (3.10) follows on $\partial S$

$$
\begin{aligned}
& \rho^{a b} c^{3}{ }_{a b}=c^{3 \perp}=\sqrt{2}, \quad \rho_{(a} e^{e} c^{3}{ }_{b) e}=c^{3 \mid}=0, \\
& \rho_{(a}^{e} \hat{\chi}_{b) e c d} \rho^{c d}=\hat{\chi}^{\perp \perp}=0, \quad \hat{\chi}^{\perp \perp}=\rho^{a b} \hat{\chi}_{a b c d} \rho^{c d}=\hat{\chi}^{\perp \perp}=0, \\
& \rho_{(a}{ }^{e} \Phi_{b) e c d} \rho^{c d}=\rho^{c d} \Phi_{c d e(a} \rho_{b)}{ }^{e}=\Phi^{\perp \perp}=0, \quad \rho^{a b} \Phi_{a b c d} \rho^{c d}=\Phi^{\perp \perp}=-2 s_{*} \text {. }
\end{aligned}
$$

The following result, to some extent suggested by Lemma 4.1, shows that the decisive properties of the normal matrix are independent of the given data. 
Lemma 5.1. For any solution of the reduced field equations (5.24)-(5.29) which takes on aS the values (5.37)-(5.39) we have, irrespective of the values taken by the field $\psi_{\text {abcd }}$, on $\mathcal{I} \cap U$

$$
\begin{aligned}
& c_{a b}(\Omega)=d_{a b}=-\sqrt{(\lambda / 6)} \rho_{a b}, \\
& c_{a b}^{3}=-\frac{1}{\sqrt{2}\left(1+\frac{1}{2} \tau^{2} s_{*}\right)} \rho_{a b},
\end{aligned}
$$

and the normal matrix $A^{3}=A^{a b} c^{3}$ ab for the part (5.33) of the boundary-adapted system is given on $\mathcal{I} \cap U$ by

$$
A^{3}=\frac{1}{1+\frac{1}{2} \tau^{2} s_{*}}\left(\begin{array}{ccccc}
-1 & 0 & 0 & 0 & 0 \\
0 & 0 & 0 & 0 & 0 \\
0 & 0 & 0 & 0 & 0 \\
0 & 0 & 0 & 0 & 0 \\
0 & 0 & 0 & 0 & 1
\end{array}\right)
$$

The propagation equations (5.25), (5.27), (5.29) imply on $\mathcal{I}$ the equations

$$
\begin{aligned}
& \partial_{\tau} c^{3}{ }_{a b}=-\hat{\chi}_{a b}{ }^{e f} c^{3}{ }_{e f}, \\
& \partial_{\tau}\left(\hat{\chi}_{a b c d} \rho^{c d}\right)=-\hat{\chi}_{a b}{ }^{e f} \hat{\chi}_{e f c d} \rho^{c d}+\Phi_{a b c d} \rho^{c d}, \\
& \partial_{\tau}\left(\Phi_{a b c d} \rho^{c d}\right)=-\hat{\chi}_{a b}{ }^{e f} \Phi_{e f c d} \rho^{c d} .
\end{aligned}
$$

This system splits into

$$
\begin{aligned}
& \partial_{\tau} c^{3 \mid}=\frac{1}{2} \hat{\chi}^{\mid \perp} c^{3 \perp}+\hat{\chi}^{\mid l} \cdot c^{3 \mid}, \\
& \partial_{\tau} \hat{\chi}^{\mid \perp}=\frac{1}{2} \hat{\chi}^{\mid \perp} \hat{\chi}^{\perp \perp}+\hat{\chi}^{\mid l} \cdot \hat{\chi}^{\mid \perp}+\Phi^{\mid \perp}, \\
& \partial_{\tau} \Phi^{\mid \perp}=\frac{1}{2} \hat{\chi}^{\mid \perp} \Phi^{\perp \perp}+\hat{\chi}^{\mid l} \cdot \Phi^{\mid \perp},
\end{aligned}
$$

from which ensues

$$
c^{3 \mid}=0, \quad \hat{\chi}^{\mid \perp}=0, \quad \Phi^{\mid \perp}=0,
$$

and

$$
\begin{aligned}
& \partial_{\tau} c^{3 \perp}=\frac{1}{2} \hat{\chi}^{\perp \perp} c^{3 \perp}+\hat{\chi}^{\perp \mid} \cdot c^{3 \mid}, \\
& \partial_{\tau} \hat{\chi}^{\perp \perp}=\frac{1}{2} \hat{\chi}^{\perp \perp} \hat{\chi}^{\perp \perp}+\hat{\chi}^{\perp l} \cdot \hat{\chi}^{\mid \perp}+\Phi^{\perp \perp}, \\
& \partial_{\tau} \Phi^{\perp \perp}=\frac{1}{2} \Phi^{\perp \perp} \hat{\chi}^{\perp \perp}+\hat{\chi}^{\perp \mid} \cdot \Phi^{\mid \perp},
\end{aligned}
$$

from which follows

$$
c^{3 \perp}=-\frac{\sqrt{2}}{1+\frac{1}{2} \tau^{2} s_{*}}, \quad \hat{\chi}^{\perp \perp}=-\frac{2 \tau s_{*}}{1+\frac{1}{2} \tau^{2} s_{*}}, \quad \Phi^{\perp \perp}=-\frac{2 s_{*}}{1+\frac{1}{2} \tau^{2} s_{*}},
$$

whence our result. 
In the following we will consider the initial boundary value problem for the boundary adapted reduced field equations with data prescribed on $S \cap U$ and $\mathcal{I} \cap U$. The normal matrix of the boundary adapted system is determined by (5.42). It follows that "maximal dissipative" boundary conditions (cf. $[9,19]$ ) are described by a condition of the form

$$
\psi_{1111}-a \psi_{0000}-c \bar{\psi}_{0^{\prime} 0^{\prime} 0^{\prime} 0^{\prime}}=d \text { on } \mathcal{I} \cap U
$$

with complex-valued functions $a, b$ on $\mathcal{I}$ satisfying

$$
|a|+|c| \leq 1
$$

and a complex-valued function $d$ on $\mathcal{I}$ which represents the freely specifiable boundary data.

Remark. The differences of the matrices $A^{01}$ in (5.32) and (5.35) and of the associated normal matrices could suggest that the freedom to prescribe boundary data is larger for the standard system of reduced equations. The uniqueness result for the solution of the conformal field equations, which is based on the use of the boundary adapted system of reduced equations shows that this cannot be true. The difference between the two types of reduced systems shows up again in the discussion of the conservation of the constraints near the boundary $\mathcal{I}$, where we obtain different systems of subsidiary equations in the two cases. In the case of the standard system the discussion of the conservation of the constraints would be quite involved. If we require that the constraints be satisfied, it should follow that the freedom we have in prescribing boundary data for the standard systems is in fact the same as that considered in the case of the boundary adapted system.

\subsection{Corner conditions}

Suppose we want to solve the initial boundary value problem for the conformal field equations with data given on $S$ and a boundary condition of the type (5.48). As is natural, we will assume that the functions $a, c$ on $\mathcal{I}$ have at least the smoothness which we want to obtain for the solution. Given intial data on $S$ near $\partial S$ in the boundary adapted gauge introduced in Section 5.3 and using the boundary adapted reduced field equations, we can determine, up to a given order $k$ which should be consistent with the smoothness properties of the data, a formal expansion of the solution on $S$ near $\partial S$ in terms of the coordinate $\tau=x^{0}$. Restricting this expansion to $\partial S$ we get in particular a formal expansion of the function $\psi_{1111}-a \psi_{0000}-c \bar{\psi}_{0^{\prime} 0^{\prime} 0^{\prime} 0^{\prime}}$, if we assume that $a, c \in C^{k}(\mathcal{I})$. It is then natural to assume, that the free datum $d$ in (5.48) is given as a function of the coordinates on $\mathcal{I}$, in particular of $\tau$, such that it allows a formal expansion up to order $k$ in $\tau$ and that this expansion coincides up to this order with the expansion of the left-hand side of (5.48) (cf. the discussion in [9]). We will refer to this requirement as to the "corner condition of order $k$ " and if the condition is satisfied to any order just as to the "corner condition". Given $C^{\infty}$ initial data on $S$ and assuming that $a, c \in C^{\infty}(\mathcal{I})$, we obtain a formal expansion of the left-hand side of (5.48) to any order. Borel's theorem tells us how to construct functions $d \in C^{\infty}(\mathcal{I})$ having the same formal expansion at $\partial S$. In the case of weak differentiability 
there arise some subtleties with the corner conditions pointed out in [19]. We have no reason to analyse these in this article. We shall show in Section 7 that we can prescribe instead of the function $d$ in (5.48) a smooth conformal structure on $\mathcal{I}$ as boundary datum. Of course, in this case we also have to observe corner conditions. It is immediately seen from the discussion in Section 7 how the corner conditions are to be expressed in terms of the conformal structure and we will not discuss this any further.

\section{The subsidiary equations}

In the following we will consider solutions to the reduced field equations on some subsets of $S \times[0, \infty$ [. As before we identify $S$ with $S \times[0]$ and $\mathcal{I}$ with $\partial S \times[0, \infty$ [. The purpose of this section is the proof of a "reduction lemma". For convenience it will be stated for smooth solutions. By the way it will be derived it will become clear, however, that it remains true also under weaker smoothness assumptions.

\section{Lemma 6.1.}

(i) Suppose $V$ is an open subset of $S$ and $U$ is an open neighbourhood of $V$ in $S \times$ $[0, \infty[$. Assume the quantities (5.22) given on $U$ represent a smooth solution of the reduced equations (standard or boundary adapted) for data on $V$ which satisfy the constraint equations. Denote by $g$ the metric for which the frame obtained from (5.22) is orthonormal and by $D^{+}(V)$ the future domain of dependence of $V$ in $U$ with respect to $g$. Then the conformal field equations are satisfied on $D^{+}(V)$ by the fields (5.22).

(ii) Suppose $p$ is a point in $\partial S, U$ is an open neighbourhood of $p$ in $S \times[0, \infty[$ and $V$ is the intersection of $U$ with $S \cup \mathcal{I}$. Assume the quantities (5.22) given on $U$ represent a smooth solution of the boundary adapted reduced field equations for data given on $V$ in the boundary adapted gauge which satisfy on $V \cap S$ the constraint equations (no assumptions are made on boundary conditions on $V \cap \mathcal{I}$ ). Denote by $g$ the metric for which the frame obtained from (5.22) is orthonormal and by $D^{+}(V)$ the future domain of dependence of $V$ in $U$ with respect to $g$. Then the conformal field equations are satisfied on $D^{+}(V)$ by the fields (5.22).

\subsection{Derivation of the subsidiary equations}

The conformal field equations read

$$
\left\langle\Sigma^{i}, c_{p} \wedge c_{q}\right\rangle=0, \quad\left\langle\Delta^{i}{ }_{k}, c_{p} \wedge c_{q}\right\rangle=0, \quad\left\langle\Delta_{k}, c_{p} \wedge c_{q}\right\rangle=0, \quad \Lambda_{a b c d}=0,
$$

where we assume the expressions

$$
\Omega^{i}{ }_{j}=\frac{1}{2} \Omega d^{i}{ }_{j k l} \sigma^{k} \wedge \sigma^{l}, \quad \Omega_{j}=\frac{1}{2}\left(-d_{i} d^{i}{ }_{j k l}\right) \sigma^{k} \wedge \sigma^{l}=-b_{i} \Omega^{i}{ }_{j}
$$

for the curvature forms, where

$$
-2 \Lambda_{a b c d} \equiv P \phi_{a b c d}-2 D_{d}^{f} \phi_{a b c d f}=-2\left(P_{a b c d}-\frac{3}{4} \varepsilon_{d(c} C_{a b)}\right),
$$


and where the functions $\Omega, d_{k}=\Omega b_{k}$ are given by (5.10), (5.11) such that Eq. (3.55) is satisfied which implies

$$
\dot{b}_{k}+b_{0} b_{k}-\frac{1}{2} \eta_{0 k}\left(b_{j} b^{j}-2 \bar{\lambda} \Omega^{-2}\right)=0 .
$$

The reduced equations are given by

$$
i_{c_{0}} \Sigma^{i}=0, \quad i_{c_{0}} \Delta_{k}^{i}=0, \quad i_{c_{0}} \Delta_{k}=0
$$

and by $P_{a b c d}=0$ in case we use the standard system respectively by (5.33) if we use the boundary adapted system. The reduced equations incorporate the gauge defined by

$$
\left\langle\sigma^{i}, c_{k}\right\rangle=\delta_{k}^{i}, \quad\left\langle\omega_{j}^{i}, c_{0}\right\rangle=0, \quad\left\langle\omega_{k}, c_{0}\right\rangle=0
$$

and they are solved for data satisfying the constraint equations on $S=\{\tau=0\}$. Consequently the solution $u=\left(c^{\mu}{ }_{k}, \Gamma_{i}{ }_{k}{ }_{k}, f_{k}, \hat{\Gamma}_{i j}, d^{i}{ }_{j k l}\right)$ of the evolution equations satisfies all equations on $S$. To show that it also satisfies all equations in the domain of dependence of $S$ with respect to the metric $g$ for which the frame $c_{k}$ is orthonormal, we derive a linear symmetric hyperbolic system of subsidiary equations for the quantities

$$
\left\langle\Sigma^{i}, c_{p} \wedge c_{q}\right\rangle, \quad\left\langle\Delta_{k}^{i}, c_{p} \wedge c_{q}\right\rangle, \quad\left\langle\Delta_{k}, c_{p} \wedge c_{q}\right\rangle, \quad p, q \neq 0, \quad C_{a b}
$$

and

$$
\begin{aligned}
& \delta_{k} \equiv b_{k}-f_{k}-\Omega^{-1} \nabla_{k} \Omega, \\
& \gamma_{j k} \equiv \hat{\Gamma}_{j k}-\hat{\nabla}_{j} b_{k}-\frac{1}{2} b_{i} S(b)_{j}{ }^{i}{ }_{k}+\bar{\lambda} \Omega^{-2} \eta_{j k}, \quad s_{[p q]},
\end{aligned}
$$

where

$$
s_{p q}=\hat{\Gamma}_{p q}-\hat{\nabla}_{p} f_{q}-\frac{1}{2} f_{k} S(f)_{p}{ }^{k}{ }_{q} .
$$

The fields (6.3), (6.4) will be called "zero quantities" in the following. It may be observed here, that the first two expressions in (6.4) may in principle be singular where $\Omega$ vanishes. It will be seen however that this creates no problems with the uniqueness property required for the subsidiary system even in the case $\lambda>0$, where we have a time-like boundary. Since the zero quantities vanish on $S$, we will be able to derive with the help of the subsidiary equations estimates which imply that the zero quantities vanish everywhere in the domain of dependence of $S$ and the boundary $\mathcal{I}$. This will show that in fact all conformal field equations (6.1) are satisfied.

We shall use the identity

$$
\begin{aligned}
\left\langle\Delta^{i}{ }_{j}, c_{p} \wedge c_{q}\right\rangle= & r^{i}{ }_{j p q}-\left\langle\Sigma^{k} ; c_{p} \wedge c_{q}\right\rangle \Gamma_{k}{ }^{i}{ }_{j}-\Omega d^{i}{ }_{j p q} \\
& -\delta^{i}{ }_{q} s_{p j}+\delta^{i}{ }_{p} s_{q j}-\delta^{i}{ }_{j}\left(s_{p q}-s_{q p}\right)+\eta^{i k}\left(s_{p k} \eta_{q j}-s_{q k} \eta_{p j}\right),
\end{aligned}
$$

where $r^{i}{ }_{j p q}$ is the curvature tensor defined by the connection coefficients $\Gamma_{i}{ }_{k}{ }_{k}$ (of which we do not know at the present stage whether they define a torsion-free connection) and the $c_{k}$. This identity corresponds to the usual decomposition of the curvature tensor into its irreducible parts and $s_{p q}$, which will be seen to be symmetric, represents the content of the 
Ricci tensor of $g$. Finally it follows by (2.39) from $\delta_{k}=0, \gamma_{j k}=0$, that the solutiun $u$ will be such that the field $\tilde{\Gamma}_{j k}$ derived from the metric $\tilde{g}=\Omega^{-2} g$ satisfies the equation

$$
\begin{aligned}
\tilde{\Gamma}_{j k}= & \hat{\Gamma}_{j k}-\hat{\nabla}_{j}\left(f_{k}+\Omega^{-1} \nabla_{k} \Omega\right)-\frac{1}{2}\left(f_{i}+\Omega^{-1} \nabla_{i} \Omega\right) S\left(f+\Omega^{-1} \nabla \Omega\right)_{j}{ }^{i}{ }_{k} \\
& +\bar{\lambda} \Omega^{-2} g_{j k}=0,
\end{aligned}
$$

which implies that $\tilde{g}$ is a solution to Einstein's vacuum field equations $\operatorname{Ric}[\tilde{g}]=\lambda \tilde{g}$.

Denoting by $L_{0}$ the Lie-derivative in the direction of $c_{0}$ we get

$$
L_{0}\left\langle\Sigma^{i}, c_{p} \wedge c_{q}\right\rangle=\left\langle L_{0} \Sigma^{i}, c_{p} \wedge c_{q}\right\rangle+\left\langle\Sigma^{i}, L_{0} c_{p} \wedge c_{q}\right\rangle+\left\langle\Sigma^{i}, c_{p} \wedge L_{0} c_{q}\right\rangle .
$$

Using the reduced equations, the definition of $\Delta^{i}{ }_{k}$, and the fact that by the way we have given $\Omega^{i}{ }_{j}$ it satisfies the Bianchi identity (2.24), we obtain

$$
L_{0} \Sigma^{i}=\left(i_{c_{0}} d+d i_{c_{0}}\right) \Sigma^{i}=i_{c_{0}} \mathrm{~d} \Sigma^{i}=i_{c_{0}}\left(\Delta_{k}^{i} \wedge \sigma^{k}-\omega_{k}^{i} \wedge \Sigma^{k}\right) .
$$

Observing that $i_{c_{0}} \Sigma^{i}=0$, we finally get for $p, q=1,2,3$

$$
\begin{aligned}
L_{0}\left\langle\Sigma^{i}, c_{p} \wedge c_{q}\right\rangle= & \left\langle\Delta^{i}{ }_{0}, c_{p} \wedge c_{q}\right\rangle-\left\langle\Sigma^{i}, c_{l} \wedge c_{q}\right\rangle\left\langle\omega_{0}^{l}, c_{p}\right\rangle \\
& -\left\langle\Sigma^{i}, c_{p} \wedge c_{l}\right\rangle\left\langle\omega_{0}^{l}, c_{q}\right\rangle .
\end{aligned}
$$

By similar arguments we derive for $p, q=1,2,3$

$$
\begin{aligned}
L_{0}\left\langle\Delta_{k}^{i}, c_{p} \wedge c_{q}\right\rangle= & -S\left(\left\langle\Delta_{l}, c_{p} \wedge c_{q}\right\rangle\right)_{0}{ }^{i}{ }_{k}-\left\langle\Lambda_{k}^{i}, c_{0} \wedge c_{p} \wedge c_{q}\right\rangle \\
& -\left\langle\omega_{0}^{l}, c_{p}\right\rangle\left\langle\Delta^{i}{ }_{k}, c_{l} \wedge c_{q}\right\rangle-\left\langle\omega^{l}{ }_{0}, c_{q}\right\rangle\left\langle\Delta^{i}{ }_{k}, c_{p} \wedge c_{l}\right\rangle, \\
L_{0}\left\langle\Delta_{k}, c_{p} \wedge c_{q}\right\rangle= & -\left\langle\Lambda_{k}, c_{0} \wedge c_{p} \wedge c_{q}\right\rangle-\left\langle\omega^{l}{ }_{0}, c_{p}\right\rangle\left\langle\Delta_{k}, c_{l} \wedge c_{q}\right\rangle \\
& -\left\langle\omega_{0}^{l}, c_{q}\right\rangle\left\langle\Delta_{k}, c_{p} \wedge c_{l}\right\rangle,
\end{aligned}
$$

where

$$
\Lambda_{k}^{i}=\mathrm{d} \Omega^{i}{ }_{k}-\Omega^{i}{ }_{j} \wedge \omega^{j}{ }_{k}+\omega^{i}{ }_{j} \wedge \Omega^{j}{ }_{k}+S\left(\Omega_{l}\right)_{j}{ }^{i}{ }_{k} \wedge \sigma^{j}
$$

and

$$
\Lambda_{k}=\mathrm{d} \Omega_{k}-\Omega_{j} \wedge \omega_{k}^{j}+\omega_{j} \wedge \Omega_{k}^{j} .
$$

By straightforward calculations we find

$$
\begin{aligned}
\Lambda_{j}^{i}= & \Omega d^{i}{ }_{j p q} \Sigma^{p} \wedge \sigma^{q}+\left\{\frac{1}{12} \Omega \nabla_{m} d^{m}{ }_{s t k} \varepsilon_{j}{ }^{i t k}{ }_{\varepsilon_{r p q}}{ }^{s}\right. \\
& \left.+\delta_{m}\left(d^{m i}{ }_{p q} \eta_{r j}-d^{m}{ }_{j p q} \eta^{i}{ }_{r}\right)\right\} \sigma^{r} \wedge \sigma^{p} \wedge \sigma^{q},
\end{aligned}
$$

and

$$
\Lambda_{k}=-b_{j} \Lambda^{j}{ }_{k}+\gamma_{i j} \sigma^{i} \wedge \Omega^{j}{ }_{k} .
$$

The right member of the first of these two equations, whence also the right member of the last equation, can be expressed as a linear expression of the zero quantities. It follows that also the right members of Eqs. (6.6)-(6.8) are linear in the zero quantities. 
We know already that $\delta_{0}=0$. The gauge conditions and Eq. (6.2) imply that $\gamma_{0 k}=0$. It follows directly from the definitions and the gauge conditions that

$$
\hat{\nabla}_{0} \delta_{p}=\gamma_{p 0}-\hat{\Gamma}_{p}^{j}{ }_{0} \delta_{j}, \quad p=1,2,3,
$$

and, using Eq. (6.2),

$$
\hat{\nabla}_{0} \gamma_{p k}=-\gamma_{q k} \hat{\Gamma}_{p}{ }^{q} 0-b_{0} \gamma_{p k}-b_{k} \gamma_{p 0}+\eta_{0 k}\left(b^{j} \gamma_{p j}-2 \bar{\lambda} \Omega^{-2} \delta_{a}\right)
$$

for $p=1,2,3$ and $k=0,1,2,3$. Using again the propagation equations we derive

$$
\begin{aligned}
\hat{\nabla}_{0} s_{[p q]}= & \frac{1}{2}\left(\left\langle\Delta_{0}, c_{p} \wedge c_{q}\right\rangle+f_{i}\left\langle\Delta^{i}{ }_{0}, c_{p} \wedge c_{q}\right\rangle\right) \\
& +\frac{1}{8}\left(\hat{\Gamma}_{p}{ }^{l}{ }_{0}\left\langle\Delta^{j}{ }_{j}, c_{l} \wedge c_{q}\right\rangle-\hat{\Gamma}_{q}{ }^{l}{ }_{0}\left\langle\Delta^{j}{ }_{j}, c_{l} \wedge c_{p}\right\rangle\right) .
\end{aligned}
$$

The derivation of the equations for $C_{a b}$ is similar to that given in [7]. Writing

$$
H_{i j k}=\nabla_{p} d_{k i j}^{p}
$$

in terms of spinors and decomposing into irreducible parts, we get the identity

$$
\begin{aligned}
& H_{a a^{\prime} b b^{\prime} c c^{\prime}}=-\tau^{d}{ }_{c^{\prime}} \Lambda_{a b c d} \varepsilon_{a^{\prime} b^{\prime}}-\tau_{c}{ }^{d^{\prime}} \bar{\Lambda}_{a^{\prime} b^{\prime} c^{\prime} d^{\prime}} \varepsilon_{a b} \\
& =-\tau^{d}{ }_{c^{\prime}}\left(P_{a b c d}-\frac{3}{4} \varepsilon_{d(c} C_{a b)}\right) \varepsilon_{a^{\prime} b^{\prime}} \\
& -\tau_{c}{ }^{d^{\prime}}\left(\bar{P}_{a^{\prime} b^{\prime} c^{\prime} d^{\prime}}-\frac{3}{4} \varepsilon_{d^{\prime}\left(c^{\prime}\right.} \bar{C}_{\left.a^{\prime} b^{\prime}\right)}\right) \varepsilon_{a b} .
\end{aligned}
$$

This implies the further identity

$$
\begin{aligned}
& P C_{a b}-\mathcal{D}_{(a}{ }^{c} C_{b) c}+2 \mathcal{D}^{e f} P_{a b e f}+2 \sqrt{2} \chi^{b}{ }_{h}{ }^{h h}\left(P_{c d b e}-\frac{3}{4} \varepsilon_{e(b} C_{c d)}\right) \\
& \quad=2 \nabla^{c c^{\prime}} \Lambda_{a b c c^{\prime}}
\end{aligned}
$$

On the other hand we get by a direct calculation which uses the symmetries of $d_{i j k l}$

$$
\begin{aligned}
\nabla^{k} H_{i j k}= & d_{l[j}{ }^{k p}\left\langle\Delta^{l}{ }_{i]}, c_{k} \wedge c_{p}\right\rangle-d_{i j}{ }^{l p}\left\langle\Delta_{l}{ }^{k}, c_{p} \wedge c_{k}\right\rangle \\
& +\frac{1}{2}\left\langle\Sigma_{k}, c_{q} \wedge c_{p}\right\rangle \nabla^{q} d_{i j}{ }^{k p}+d_{p l i j} s^{[p l]} .
\end{aligned}
$$

From (6.14) to (6.16) follows the equation for $C_{a b}$, the right member of which is again linear in the zero quantities. That the equation is symmetric hyperbolic is seen immediately in the case, where the standard system has been used for the evolution. In this case (6.15) reduces to

$$
P C_{a b}-\mathcal{D}_{(a}{ }^{c} C_{b) c}-\frac{3}{\sqrt{2}} \chi_{d}^{e}{ }^{f d_{\varepsilon_{f(a l}} C_{b e)}}=2 \nabla^{c c^{\prime}} H_{a b c c^{\prime}}
$$

In case the boundary adapted system has been used for the propagation, Eq. (6.15) can be written in the form

$$
\begin{aligned}
& P C_{00}+\mathcal{D}_{00} C_{01}=U_{00}, \quad P C_{01}+\mathcal{D}_{00} C_{11}-\mathcal{D}_{11} C_{00}=U_{01}, \\
& P C_{11}-\mathcal{D}_{11} C_{01}=U_{11}
\end{aligned}
$$

with linear functions $U_{a b}$ of the zero quantities. 
Eqs. (6.6)-(6.8), (6.11)-(6.13), (6.15), with the right-hand sides written as linear expressions in the zero quantities as explained above, constitute the system of subsidiary equations. It is an important property of this system that Eqs. (6.11), (6.12) decouple from the remaining subsidiary equations and form a linear system of ordinary differential equations. Since the zero quantities vanish on $S$, the functions $\delta_{k}, \gamma_{j k}$ vanish on the set $\{\Omega>0\}$. The remaining part of the subsidiary system then has coefficients which extend continuously to $\mathcal{I}$. It is symmetric hyperbolic and its cone of characteristic rays is inside the null cone defined by the metric $g$. This implies, irrespective of the sign of $\lambda$, that the zero quantities vanish in the domain of dependence of a given subset of $S$ with respect to the metric determined by the solution of the reduced equations. Thus the conformal field equations are satisfied in that region. Near a time-like boundary as represented by $\mathcal{I}$ the argument is slightly more involved.

\subsection{Conservation of constraints near a time-like boundary}

In case the boundary adapted gauge and system of reduced equations have been used to propagate the fields near the boundary $\mathcal{I}$, the subsidiary equations can be written with respect to conformal Gauss coordinates near a given point on $\partial S$ in the form

$$
F^{\mu} \nabla_{\mu} u=G u
$$

where $\nabla$ is the Levi-Civita connection for $g$ and $F^{\mu}, G$ are matrix-valued functions such that the $F^{\mu}$ are hermitian, $F^{\mu} \nabla_{\mu} x^{0}$ is positive definite near $\partial S$, and, as follows from (6.17) and Lemma 5.1, the normal matrix of the subsidiary system satisfies the decisive condition

$$
F^{3}=0 \quad \text { on } \mathcal{I} \cap \mathcal{U}
$$

Furthermore, we find that

$$
\operatorname{det}\left(F^{\mu} \xi_{\mu}\right)=\left(\tau^{\mu} \xi_{\mu}\right)^{p}\left(\left(\tau^{\mu} \xi_{\mu}\right)^{2}+2 \xi_{00} \xi_{11}\right) \geq\left(\tau^{\mu} \xi_{\mu}\right)^{p} g^{\mu v} \xi_{\mu} \xi_{v}
$$

with some positive integer $p$. Let now $t>0$ be a smooth function defined in the neighbourhood $U$ of $\partial S$ where we solved the boundary adapted reduced equations. We assume that each level set of $t$ intersects $S$ as well as $\mathcal{I}$ in space-like surfaces, that these intersections approach a closed subset of $\partial S$ as the function $t$ approaches 0 , and that the gradient of $t$ with respect to the metric obtained from the solution to the propagation equations is time-like and lies in a compact region of the interior of the future null cone. For $a \in \mathbb{R}, a \geq 0$ we set $T_{a}=\{p \in M \mid t(p)=a\}, V_{a}=\{p \in M \mid 0 \leq t(p) \leq a\}$ and $I_{a}=\mathcal{I} \cap V_{a}, S_{a}=S \cap V_{a}$ Fix a small $A>0$. Integrating $\nabla_{\mu}\left({ }^{t} \bar{u} F^{\mu} u\right)={ }^{t} \bar{u} H u$ with $H={ }^{t} \bar{G}+G+\nabla_{\mu} F^{\mu}$ over $V_{a}$ for $a \in] 0, A]$, observing (6.18), our assumptions on the function $t$, and the fact that $u=0$ on $S_{a}$, we get after a partial integration

$$
\int_{T_{a}} n_{\mu}\left({ }^{t} \bar{u} F^{\mu} u\right) \mathrm{d} S_{a}=\int_{V_{a}}\left({ }^{t} \bar{u} H u\right) \mathrm{d} \mu_{g} \leq C \int_{0}^{a}\left(\int_{T_{s}} n_{\mu}\left({ }^{t} \bar{u} F^{\mu} u\right) \mathrm{d} S_{s}\right) \mathrm{d} s,
$$


where $C \geq 0$ is a constant which does not depend on $a, \mathrm{~d} S_{s}$ denotes the volume element induced on $T_{s}$ by $g$, and $n_{\mu}$ is the future directed unit normal to $T_{s}$. Gronwall's lemma implies now, that $u=0$ on $V_{A}$, which we wanted to show.

\section{Covariant boundary conditions}

The discussion in Section 5 suggests looking for solutions of initial boundary value problems for the conformal field equations where the boundary data are given in the form (5.48). It appears, however, that boundary conditions of this general form are not suitable for geometric discussions. On $\mathcal{I}$ the frame vector field $c_{3}=n$ normal to the boundary is singled out naturally. We have prescribed initial conditions for the conformal geodesic curves everywhere on $S$, which fix in particular the time-like vector field $c_{0}$ on $\mathcal{I}$. Rotations of the frame on $\mathcal{I}$ which leave the normal vector and the time-like vector field on $\mathcal{I}$ unchanged are given by $c_{a a^{\prime}} \rightarrow e^{\left(a^{\prime}-a\right) i \Phi} c_{a a^{\prime}}$. Under these rotations the components of the rescaled Weyl spinor transform according to $\psi_{a b c d} \rightarrow e^{(2-k) i \Phi} \psi_{a b c d}$ with $k=a+b+c+d$. Thus the boundary condition (5.48) and condition (5.49) can only be given an invariant meaning under such transformations if we require the transformation behaviour $a \rightarrow e^{-4 i \Phi} a, c \rightarrow$ $c, d \rightarrow e^{-2 i \Phi} d$. We shall restrict ourselves to boundary conditions, where the functions $a$, $c$ are constant. This is only sensible, if we assume that $a$ vanishes. Thus we shall consider boundary conditions of the form

$$
\psi_{1111}-c \bar{\psi}_{0^{\prime} 0^{\prime} 0^{\prime} 0^{\prime}}=d, \quad c=\text { const } ., \quad|c| \leq 1 .
$$

Note that the assumption, that the data correspond to fields given in a boundary adapted gauge, is part of their specification. In general there does not exist a boundary adapted gauge in a complete neighbourhood of $\partial S$ in which the quantity $d$ could be given. Therefore it is important that the transformations of $d$ under changes of the boundary adapted gauges are controlled in terms of the changes on $\partial S$. Consider in a fixed boundary adapted gauge the function $d=d\left(\tau, x^{1}, x^{2}\right)$ along a given conformal geodesic on $\mathcal{I}$ which is specified by the values of the coordinates $x^{1}, x^{2}$ at points of $\partial S$. A change to another boundary adapted gauge implies a change of the frame field $c_{a a^{\prime}} \rightarrow e^{\left(a^{\prime}-a\right) i \Phi\left(x^{1}, x^{2}\right)} c_{a a^{\prime}}$ on $\partial S$. As a consequence of the conformal geodesic equation (cf. (2.41)) this entails the transformation $d\left(\tau, x^{1}, x^{2}\right) \rightarrow e^{-2 i \Phi\left(x^{1}, x^{2}\right)} d\left(\tau, x^{1}, x^{2}\right)$, i.e. the phase factor remains constant along the geodesic. Thus $d$ has to be given as a spin weighted quantity on $\mathcal{I}$ which has a transformation law that is correlated to transformations of the data on $S$ as described above.

Still, there is no obvious way to compare such boundary conditions for different choices of the frame if the field $c_{0}$, which in general cannot be fixed by a natural requirement, is also changed. In the following we shall study an example of a boundary condition, which has a covariant meaning.

\subsection{Boundary conditions in terms of fields on $\mathcal{I}$}

We assume again that $n=c_{3}$ is the inward pointing unit normal on $\mathcal{I}$ and use the spinor 


$$
\rho^{a a^{\prime}}=\varepsilon_{0}{ }^{a} \varepsilon_{0^{\prime}} a^{\prime}-\varepsilon_{1}^{a} \varepsilon_{1^{\prime}}^{a^{\prime}}
$$

to project fields into $\mathcal{I}$ in analogy to the splittings we performed with respect to the time-like spinor $\tau^{a a^{\prime}}$. We set $\tau_{a b}=\rho_{b}{ }^{b^{\prime}} \tau_{a b^{\prime}}=2 \varepsilon_{(a}{ }^{0} \varepsilon_{b)}{ }^{1}$. The $n$-magnetic and $n$-electric parts of the rescaled Weyl tensor $d_{a a^{\prime} b b^{\prime} c c^{\prime} d d^{\prime}}=\phi_{a b c d} \varepsilon_{a^{\prime} b^{\prime}} \varepsilon_{c^{\prime} d^{\prime}}+\bar{\phi}_{a^{\prime} b^{\prime} c^{\prime} d^{\prime}} \varepsilon_{a b} \varepsilon_{c d}$, which represent tensor fields in $\mathcal{I}$, are given by

$$
B_{a b c d}=\frac{1}{2} \rho_{b}{ }^{\prime} \rho^{e e^{\prime}} \rho_{d}^{c^{\prime}} \rho^{f f^{\prime}} d_{a a^{\prime} e e^{\prime} c c^{\prime} f f^{\prime}}^{*}=-\frac{1}{2} i\left(\phi_{a b c d}-\phi_{a b c d}^{+}\right)
$$

respectively

$$
E_{a b c d}=\frac{1}{2} \rho_{b}{ }^{\prime} \rho^{e e^{\prime}} \rho_{d}^{c^{\prime}} \rho^{f f^{\prime}} d_{a a^{\prime} e e^{\prime} c c^{\prime} f f^{\prime}}=\frac{1}{2}\left(\phi_{a b c d}+\phi_{a b c d}^{+}\right),
$$

where the star denotes again the four-dimensional dual and

$$
\phi_{a b c d}^{+}=\rho_{a}{ }^{\prime} \rho_{b}{ }^{\prime} \rho_{c}{ }^{c^{\prime}} \rho_{d d} d^{\prime} \bar{\phi}_{a^{\prime} b^{\prime} c^{\prime} d^{\prime}}
$$

the $\rho$-conjugated of $\phi_{a b c d}$. The magnetic part admits a decomposition

$$
B_{a b c d}=b_{a b c d}+b_{a b} \tau_{c d}+\tau_{a b} b_{c d}+\frac{1}{4} b\left(3 \tau_{a b} \tau_{c d}-2 \varepsilon_{a(c} \varepsilon_{d) b}\right),
$$

where

$$
b_{a b c d}=b_{(a b c d)}, \quad \tau^{a b} b_{a b c d}=0, \quad b_{a b}=b_{(a b)}, \quad \tau^{a b} b_{a b}=0, \quad b=\bar{b} .
$$

A similar decomposition is obtained for the electric part. The spinor field $b_{a b c d}$ on $\mathcal{I}$, which represents the trace free part of the projection of the magnetic part into the space orthogonal to $\tau^{a a^{\prime}}$, has as non-vanishing components only

$$
b_{1111}=-\frac{1}{2} i\left(\phi_{1111}-\bar{\phi}_{0^{\prime} 0^{\prime} 0^{\prime} 0^{\prime}}\right)=B_{1111}
$$

and $b_{0000}$, which is the complex conjugate of $b_{1111}$. Also $b_{11}=-\frac{1}{2} i\left(\phi_{0111}-\bar{\phi}_{1^{\prime} 0^{\prime} 0^{\prime} 0^{\prime}}\right)$ is the complex conjugate of $b_{00}, b_{01}=0, b=-i\left(\phi_{0011}-\bar{\phi}_{1^{\prime} 1^{\prime} 0^{\prime} 0^{\prime}}\right)$. Similarly the field $e_{a b c d}$ is completely represented by the component $e_{1111}=\frac{1}{2}\left(\phi_{1111}+\bar{\phi}_{0^{\prime} 0^{\prime} 0^{\prime} 0^{\prime}}\right)=E_{1111}$. From this we find that some of the boundary conditions (7.1) can be expressed with respect to tensor fields in $\mathcal{I}$. In case $c=1$ condition (7.1) can be replaced by

$$
B_{1111}=d,
$$

while in the case $c=-1$ we can write

$$
E_{1111}=d
$$

with suitable data $d$.

To relate such boundary conditions given with respect to different choices of $c_{0}$, we try to exploit the special properties of the conformal boundary. We assume that we are given a solution of the conformal field equations which is such that either condition (7.2) or condition (7.3) is satisfied with some given function $d$ on $\mathcal{I}$. We denote by $D_{a b}, T$, and $\delta_{a b}$ the inner covariant derivative on $\mathcal{I}$ in the direction of $\rho_{(b}{ }^{\prime} c_{a) a^{\prime}}, \tau^{a a^{\prime}} c_{a a^{\prime}}$ and $\tau_{(a}{ }^{e} c_{b) e}$ respectively, such that we have $D_{a b}=\frac{1}{2} \tau_{a b} T+\delta_{a b}$. We know then from (4.11), that 
$D^{a b} B_{a b c d}=0, D^{a b} E_{a b c d}=0$ on $\mathcal{I}$. Using the expansions above for $D_{a b}$ and $B_{a b c d}$, we find that the first of these equations is equivalent to

$$
\begin{aligned}
\frac{1}{2} T b+\delta^{a b} b_{a b}= & \frac{1}{2} b_{a b} T \tau^{a b}-\frac{3}{4} b D^{a b} \tau_{a b}+\frac{1}{2} \tau^{e f} D^{a b} b_{a b e f}, \\
2 T b_{c d}+\delta_{c d} b= & 2\left(b_{c d} D^{e f} \tau_{e f}+b_{e f} D^{e f} \tau_{c d}\right) \\
& -\frac{3}{2} b T \tau_{c d}+\left(2 \varepsilon_{c}^{e} \varepsilon_{d}^{f}+\tau_{c d} \tau^{e f}\right) D^{a b} b_{a b e f} .
\end{aligned}
$$

For given $b_{a b c d}$, i.e. for given datum (7.2), we can read these equations as partial differential equations for the fields $b_{a c}, b$. The principal part of the system is given explicitly by writing the system (7.4), (7.5) in the form

$$
\left(\begin{array}{ccc}
\frac{1}{2} \tau^{\mu} & -c_{10^{\prime}}^{\mu} & -c_{01^{\prime}}^{\mu} \\
-c_{01^{\prime}}^{\mu} & 2 \tau^{\mu} & 0 \\
-c_{10^{\prime}}^{\mu} & 0 & 2 \tau^{\mu}
\end{array}\right) \partial_{\mu}\left(\begin{array}{c}
b \\
b_{00} \\
b_{11}
\end{array}\right)=\left(\begin{array}{c}
f_{0} \\
f_{1} \\
f_{2}
\end{array}\right),
$$

where $\tau^{\mu}=\tau^{a a^{\prime}} c^{\mu}{ }_{a a^{\prime}}$ and the right-hand side depends linearly on $b_{a c}, b$ and on the "source term" $D^{a b} b_{a b e f}$. Thus the equations form a linear symmetric hyperbolic system.

We could use it to determine $B_{a b c d}$ on $\mathcal{I}$ from known data on $\partial S$ and the boundary data (7.2) if we knew the interior metric on $\mathcal{I}$. We could then compare boundary conditions given with respect to different gauges. A similar statement is true for the electric part of the rescaled Weyl tensor. There is a basic difference between the electric and the magnetic part. By (4.11) the magnetic part $B_{a b c d}$ is essentially given by the Cotton tensor of the interior metric $h$ on $\mathcal{I}$. This offers a possibility to relate the data (7.2) to the metric $h$. The following result, expressed in terms of the notation used in Lemma 6.1, allows, to express the boundary conditions purely in terms of the interior conformal structure on $\mathcal{I}$ which is not subject to any constraint except the requirement that corner conditions be satisfied.

Lemma 7.1. Suppose the fields (5.22) represent a solution of the conformal field equations with cosmological constant $\lambda>0$ on $S \times[0, a[, a>0$, for which $\mathcal{I}=\partial S \times[0, a[$ represents the conformal boundary at space-like and null infinity, $g$ denotes the metric for which the frame supplied by (5.22) is orthonormal, and $h$ is the interior metric induced by $g$ on $\mathcal{I}$. We assume that the gauge considered in Section 5 can be extended to all of $S \times[0, a[$.

(i) If we know on $\partial S$ the data implied by (5.22) in the boundary adapted gauge and if we know the conformal class of $h$ on $\mathcal{I}$, we can calculate on $\mathcal{I}$ the datum (7.2) with respect to the gauge considered in Section 5.

(ii) Conversely, given on $\partial S$ the data implied by (5.22) and on $\mathcal{I}$ the data (7.2), both in the conformal gauge considered in Section 5, the conformal class of $h$ on $\mathcal{I}$ is determined uniquely as a solution of a quasi-linear symmetric hyperbolic system of partial differential equations on $\mathcal{I}$.

To show (i) we recall that by Lemma 4.1 the gauge can be determined on $\mathcal{I}$ for given data on $\partial S$ by solving the conformal geodesics equations defined by the interior conformal structure of $\mathcal{I}$. With respect to this gauge we can calculate the Cotton tensor, which by 
(4.11) determines the magnetic part of the rescaled Weyl tensor on $\mathcal{I}$. From this we can read off the desired datum (7.2).

The demonstration of the second part is more complicated. In dimension $n=3$ the curvature of the normal conformal Cartan connection is represented by $\Omega_{j}$ alone, since by (3.18) a trace free tensor possessing the symmetries of a curvature tensor vanishes. It follows then from (2.32) that $K_{j k l}$ has a tensorial transformation law under transformations $t \in H(p, q)$. This tensor, the Cotton tensor, vanishes if and only if the conformal structure can locally be represented by a flat metric. All algebraic properties which are obtained if $K_{j k l}$ is represented with respect to the Levi-Civita connection $\nabla$ of a metric $g$ hold in any representation. In addition to its antisymmetry in the last pair of indices and the property (2.30), it satisfies $K^{i}{ }_{i l}=0$. If $\varepsilon_{i j k}$ denotes the totally antisymmetric Levi-Civita symbol with respect to $g$ we have a representation

$$
K_{j k l}=K_{j i} \varepsilon_{k l}
$$

with a symmetric and trace-free tensor field $K_{j i}$. We consider the system

$$
\begin{aligned}
& 0=\Sigma^{i} \equiv \mathrm{d} \sigma^{i}+\omega_{k}^{i} \wedge \sigma^{k}, \\
& 0=\Delta_{k}^{i} \equiv \mathrm{d} \omega_{k}^{i}+\omega^{i}{ }_{l} \wedge \omega_{k}^{l}-S\left(\omega_{p}\right)_{q}{ }_{k}{ }_{k} \wedge \sigma^{q}, \\
& 0=\Delta_{k} \equiv \mathrm{d} \omega_{k}+\omega_{i} \wedge \omega_{k}^{i}-\Omega_{k}, \\
& 0=\Lambda_{j} \equiv \mathrm{d} \Omega_{j}-\Omega_{k} \wedge \omega_{j}^{k},
\end{aligned}
$$

provided by the structure equations and the fourth Bianchi identity (2.31).

We want to determine a frame field $c_{j}, j=0,1,2$ on $\mathcal{I}$ which coincides on $\partial S$ with the frame supplied by the data and satisfies the gauge conditions

$$
\left\langle\sigma^{i}, c_{k}\right\rangle=\delta_{k}^{i}, \quad\left\langle\omega_{j}^{i}, c_{0}\right\rangle=0, \quad\left\langle\omega_{k}, c_{0}\right\rangle=0,
$$

which by Lemma 4.1 corresponds to the gauge used in Section 5. Writing $\Omega_{j}=\frac{1}{2} K_{j i} \varepsilon^{i}{ }_{k l} \sigma^{k} \wedge$ $\sigma^{l}$, we evaluate the system

$$
i_{c_{0}} \Sigma^{i}=0, \quad i_{c_{0}} \Delta_{k}^{i}=0, \quad i_{c_{0}} \Delta_{k}=0, \quad \Lambda_{j}=0,
$$

in terms of the frame $c_{j}$ and coordinates $x^{\alpha}, \alpha=0,1,2$, satisfying $\left\langle\mathrm{d} x^{\alpha}, c_{0}\right\rangle=\delta_{0}^{\alpha}, x^{0}=0$ on $\partial S$. The first three of these equations involve only the derivative operator $c_{0}$. The last equation takes in terms of the Levi-Civita connection $\nabla$ of the metric for which the frame $c_{j}$ is orthonormal, the form

$$
0=\nabla^{i} K_{i j}
$$

By (4.11) certain components of the tensor field $K_{i j}$ can be expressed by the data (7.2). After these components have been given, the system obtained from (7.10) constitutes by (7.4), (7.5) a symmetric hyperbolic system. Given the data on $\partial S$, the (unique) solution of this system (cf. [20]) close to $\partial S$ determines the conformal structure on $\mathcal{I}$. In fact, we get the frame in the gauge considered in Section 5. By arguments similar to those used in the 
derivation of the subsidiary system it follows that the forms $\sigma^{i}, \omega^{i}{ }_{k}, \omega_{j}, \Omega_{l}$ supplied by the solution to (7.10), which satisfy (7.6)-(7.9) on $\partial S$, satisfy in fact (7.6)-(7.9).

\subsection{Description of the boundary data in terms of the metric $\tilde{g}$}

Conformal geodesics allow a convenient description of the covariant boundary condition studied above in terms of fields directly related to the metric $\tilde{g}$. We begin with a few observations concerning conformal geodesics in terms of the metric $\tilde{g}$ which satisfies the Einstein equation (3.1). Suppose that $x(\tau), b(\tau), c_{k}(\tau)$ are as in Lemma 3.1. We reparametrize these quantities by the arclength $\sigma$ of $x(\tau)$ with respect to $\tilde{g}$ and write $\dot{x} \equiv(\mathrm{d} x / \mathrm{d} \sigma)=\dot{x} \dot{\tau}$, $\dot{\Theta}=\dot{\Theta} \dot{\tau}$, etc. We can assume that $\dot{\tau}=\Theta$ and we have along the conformal geodesic

$$
\tilde{\nabla}_{\dot{x}} \dot{x}=y
$$

with some vector field $y$ orthogonal to $x$, which satisfies by Eq. (2.49)

$$
b=y+\dot{\Theta} \dot{x}=y+\langle b, \dot{x}\rangle \dot{x} .
$$

From this and Eq. (2.50) follows $\tilde{\nabla}_{\tilde{x}} y=-\tilde{g}(y, y) \tilde{x}$, which entails that $\tilde{g}(y, y)$ is constant along the conformal geodesic. Since $\dot{x}$ is time-like, there can occur two cases. If $b$ is proportional to $\dot{x}$ at one point it remains so and the conformal geodesic is up to a reparametrization a metric geodesic. If $y \neq 0$ at some point, this property will also be preserved and $x(\sigma)$ is a curve of non-vanishing acceleration $y$.

Setting $a_{k}=\Theta c_{k}$, we obtain a frame which is orthonormal for $\tilde{g}$. It satisfies by (2.51) the equation $\tilde{\nabla}_{\dot{x}} a_{k}=-\left\langle b, a_{k}\right\rangle \hat{x}+\left(a_{k}, \dot{x}\right) b$ which, in view of (7.12), (7.13), can be rewritten in the form $\tilde{\nabla}_{\dot{x}} a_{k}+\tilde{g}(\dot{x}, \dot{x})\left\{\tilde{g}\left(a_{k}, \tilde{\nabla}_{\dot{x}} \dot{x}\right) \dot{x}-\tilde{g}\left(a_{k}, \dot{x}\right) \tilde{\nabla}_{\dot{x}} \dot{x}\right\}=0$, showing that the frame $a_{k}$ is Fermi-transported. It follows that the coefficients $x^{k}, y^{k}$ of the expansion $\dot{x}=x^{k} a_{k}, y=$ $y^{k} a_{k}$ are constant along the conformal geodesic.

We write now $\Theta=\Omega$ and describe our gauge conditions in terms of $x(\sigma), y(\sigma)$, the conformal factor $\Omega$ and its differential $\mathrm{d} \Omega$ on $\tilde{S}$, which is known by (5.4). The smooth congruence of conformal geodesic curves in $\tilde{M}$ is obtained by solving the equations

$$
\tilde{\nabla}_{\dot{x}} \dot{x}=y, \quad \tilde{\nabla}_{\dot{x}} y=-\tilde{g}(y, y) \dot{x},
$$

with initial conditions $x(0) \in \tilde{S}, \dot{x}(0)$ is future directed and orthogonal to $\tilde{S}, \tilde{g}(\dot{x}(0)$, $\dot{x}(0))=1, y^{\mu}(0)=\left.\left(b^{\mu}-\dot{\Omega} \dot{x}^{\mu}\right)\right|_{S}=\Omega^{-1} \tilde{g}^{\mu \nu} \nabla_{\nu} \Omega$ on $\tilde{S}$. It follows that $\tilde{g}(\dot{x}, \dot{x})=1$, $\tilde{g}(\tilde{x}, y)=0, \tilde{g}(y, y)=$ const. $=-\frac{1}{3} \lambda+2 \Omega_{*} s_{*}$ along these curves in $\tilde{M}$. For $\omega \in \mathbb{R}$, $0<\omega \ll 1$ the set $\tilde{S}_{\omega}=\{\Omega=\omega\}$ defines a smooth hypersurface in $\tilde{S}$ near space-like infinity. The conformal geodesics starting from $\tilde{S}_{\omega}$ generate a smooth time-like hypersurface $T_{\omega}$ in $\tilde{M}$ near $\tilde{S}$. We denote the metric induced by $\tilde{g}$ on $T_{\omega}$ by $\tilde{h}_{\omega}$ and the function induced by $\Omega$ on $T_{\omega}$ by $\Omega_{\omega}$. Then the boundary data given on $\mathcal{I}$ are apparently represented by the conformal class of the metric which is obtained as a limit $h=\lim _{\omega \rightarrow 0} \Omega_{\omega}^{2} \tilde{h}_{\omega}$. Care must be taken here with the limit procedure. A point $p$ on $T_{\omega}$ is parametrized by the point $p^{\prime}$ where the conformal geodesic through $p$ meets $\tilde{S}_{\omega}$ and by the value at $p$ of the parameter $\sigma$ on this conformal geodesic. We require that the limit is taken along the curve, for which 
$p^{\prime}$ follows the flow generated by the field $y$ on $\tilde{S}$ and for which the value of the parameter $\tau=\tau(\sigma)$ is constant. From (5.10) follows that the parameter $\tau$ is related to the parameter $\sigma$ by

$$
\Omega_{*} \sigma=\int_{0}^{\tau} \frac{\mathrm{d} \tau^{\prime}}{1-\frac{1}{2} \tau^{\prime 2} s_{*}}
$$

This equation determines the way in which we have to let $\sigma$ go to infinity in order to achieve a limit with constant value $\tau>0$. Along any such curve we consider for a given value of $\omega$ the set of future directed null vectors $N$ of $\tilde{h}_{\omega}$ satisfying the normalization condition $\tilde{h}_{\omega}(\dot{x}, N)=\Omega_{\omega}^{-1}$. In suitably chosen coordinates these sets define limiting null cones which in turn determine the conformal structure on $\mathcal{I}$.

\section{Existence results}

The reduced conformal field equations will be used now to prove Theorem 1.1. We shall adopt the notation used in Theorem 1.1. The unknowns in the reduced field equations will be denoted in the following collectively by the "column vector"

$$
u=\left(c_{a b}^{\mu}, \Gamma_{a b c d}, \Phi_{a c b d}, \psi_{a c b d}\right) .
$$

We assume that $(\tilde{S}, \tilde{h}, \tilde{\chi})$ is a smoothly conformally compactifiable Cauchy data set for the Einstein equations (1.1) with positive cosmological constant $\lambda>0$. As described in Section 5.1 we can derive from it the data for the fields comprised by $u$. We assume that these data extend smoothly to $S$ and denote them collectively as $u_{0}$. We assume furthermore on $\mathcal{I}=\partial S \times[0, \infty[$ a smooth spin weighted field $d$ which is defined with respect to any boundary adapted gauge of the data $u_{0}$ on $S$ such that the correlation of the transformation laws for the data on $S$ and of $d$ hold as described in Section 7. Finally we assume that the data $u_{0}$ on $S$ and $d$ on $\mathcal{I}$ satisfy the corner condition discussed in Section 5.4. When we use the reduced field equations there remains the freedom to choose the frame and the local coordinates on $S$. Observing the remarks following (5.8) and (5.9), we note that the function $x^{0}=\tau$ will be independent of this choice and depend only on the metric $g$ on $S$ and on the conformal structure defined by the solution $g$ of the conformal field equations which we want to construct. This allows to identify the function $\tau$ with the projection of $M=S \times[0, \infty[$ onto $[0, \infty[$. We will now prove a result which is somewhat more general than Theorem 1.1. Observing Lemma 7.1 it is easily seen that the following theorem entails Theorem 1.1.

Theorem 8.1. Suppose we are given data $u_{0}$ on $S$ and $d$ on $\mathcal{I}$ as described above and $a$ complex number $c$ with $|c| \leq 1$. Then there exists for some $\alpha>0$ a unique smooth solution $u$ of the conformal Einstein equations (3.33) on $M_{\alpha}=\{p \in M \mid 0 \leq \tau(p)<\alpha\}$ with the properties: (i) on $S$ the data $u_{0}$ are induced by $u$, (ii) the boundary condition (7.1) is satisfied on $\mathcal{I} \cap M_{\alpha}$, (iii) in a local gauge and in conformal Gauss coordinates as described 
in Section 5.1, the fields $\Omega$ and $d_{a b}$ in (3.33) are given by (5.10), (5.23), and $\tau$ is a parameter of the conformal geodesics.

The uniqueness of the solution can be deduced from the uniqueness properties of our gauge conditions and from well-known properties of symmetric hyperbolic systems. We shall not further comment on it.

Due to the hyperbolicity of the equations this problem can be localized. Given the restriction of the data $u_{0}$ to an open subset $V \subset \tilde{S}$ on which the local gauge described in Section 5.1 can be defined, it follows from well-known results on symmetric hyperbolic systems (cf. [20]) that there exists on a certain neighbourhood $U$ of $V$ in $M$ a unique solution to the reduced field equations (5.24)-(5.30), which takes the given values on $V$. By Lemma 6.1 this is in fact a solution to the conformal field equations given in the gauge of Section 5.1 and with $\Omega$ and $d_{a b}$ given by (5.10), (5.23). Suppose we can show the existence of a unique local solution in an open neighbourhood $U$ in $M$ of a given point $p \in \partial S$ to the mixed problem for the boundary adapted system of reduced field equations, where the initial data are given by the restriction of $u_{0}$ to $V^{\prime}=S \cap U$ and the boundary conditions (7.1) hold on $V^{\prime \prime}=\mathcal{I} \cap U$. Again by Lemma 6.1 this solution solves in fact the conformal Einstein equations. The local solutions so obtained can be patched together if their initial data are given on intersecting domains (cf. [7]). Since $S$ can be covered by a finite number of local solutions we obtain as the result of the patching procedure a solution as described in Theorem 8.1.

This reduces the proof of Theorem 8.1 to the discussion of the local mixed problem. We assume that in a neighbourhood $V^{\prime}$ of the point $p \in \partial S$ in $S$ the data are given in a fixed boundary adapted gauge such that $x^{\mu}(p)=0$ and that $\tau=x^{0}, x^{1}, x^{2}$ are coordinates on $V^{\prime \prime}$. The neighbourhood $U$ of $p$ is thought of as being embedded via the coordinates onto an open neighbourhood of the origin in $\mathbb{R}^{4}$. We reformulate now our problem as a mixed problem, where the equation is a real equation of the form

$$
A^{\mu} \partial_{\mu} u=F\left(a, \partial_{v} a, u\right) \text { on } N_{\alpha}=\left\{x \in \mathbb{R}^{4} \mid 0<x^{3}, 0<x^{0}<\alpha\right\}
$$

for an $\mathbb{R}^{N}$-valued unknown $u$. Here $a$ denotes an $\mathbb{R}^{N^{\prime}}$-valued function of compact support in $\left\{x \in \mathbb{R}^{4} \mid x^{0}, x^{3} \geq 0\right\}, A^{\mu}=A^{\mu}(a, u)$ are smooth, symmetric, $N \times N$-matrix-valued functions of $(u, a)$ such that $A^{0}(a, u)$ is positive definite for $u \in \mathbb{R}^{N}$ and $F$ denotes a smooth $\mathbb{R}^{N}$-valued function. The boundary conditions are of the form

$$
B u=0 \text { on }\left\{x^{3}=0,0 \leq x^{0} \leq \alpha\right\}
$$

with a constant real $2 \times N$ matrix $B$ of rank 2 , and the initial condition is

$$
u=0 \quad \text { on }\left\{x^{3} \geq 0, x^{0}=0\right\} .
$$

This formulation is obtained by going through the following steps, where we assume for simplicity that $\alpha=\infty$ :

(i) The part of the boundary adapted reduced field equations (5.33) has the form

$$
\left(I+A^{0}\right) \partial_{\tau} \psi+A^{\alpha} \partial_{\alpha} \psi=B(\Gamma, \psi)
$$


with $A^{\mu}=A^{a b} c^{\mu}, \mu=0,1,2,3$. We replace in this equation $A^{0}$ by $A^{a b} C_{a b}$ where the smooth map $C_{a b}=C_{a b}\left(c^{0}{ }_{c d}\right)$, defined for fields $c^{0}{ }_{a b}$ satisfying the usual reality conditions, is constructed as follows. We choose smooth real functions $f_{01}, f_{10}$ on the real line and complex functions $f_{00}, f_{11}$ on the complex plane, all with compact support and such that $f_{01}=f_{10}, f_{11}(z)=-\overline{f_{00}(-\bar{z})}, f_{a b}(z)=z$ for $|z|<\gamma^{\prime}$ and $\left|f_{a b}(z)\right| \leq \gamma$ with certain constants $\gamma>\gamma^{\prime}>0$. For fixed indices $a b$ the map $C_{a b}$ is assumed to depend only on $c^{0}{ }_{a b}$ such that $C_{a b}\left(c^{0}{ }_{c d}\right)=f_{a b}\left(c^{0}{ }_{a b}\right)$. Then $C_{a b}=c^{0}{ }_{a b}$ for $\left|c^{0}{ }_{a b}\right|<\gamma^{\prime},\left|C_{a b}\right| \leq \gamma$, and the matrix $A^{a b} C_{a b}$ is hermitian. We choose the constants $\gamma, \gamma^{\prime}$ such that $I+A^{a b} C_{a b} \geq \frac{1}{2} I$. Observing that the data for $c^{0}{ }_{a b}$ vanish on $\{\tau=0\}$, we see that the new equations are equivalent to the old equations in regions where $\left|c^{0}{ }_{a b}\right|<\gamma^{\prime}$.

(ii) We choose a function $q \in C^{\infty}(\mathbb{R}, \mathbb{R})$ with $q^{\prime} \leq 0, q(s)=1$ for $s<1, q(s)=0$ for $s>2$ and define $q_{\varepsilon} \in C_{0}^{\infty}\left(\mathbb{R}^{4}, \mathbb{R}\right)$ by $q_{\varepsilon}(x)=q(|x| / \varepsilon)$ with some $\varepsilon>0$ such that

$$
\left\{x \in \mathbb{R}^{4} \mid x^{0}, x^{3} \geq 0\right\} \cap \operatorname{supp} q_{4 \varepsilon} \subset V^{\prime} \times\left[0, \infty\left[, \quad \frac{1}{2} \leq 1+\frac{1}{2} \tau^{2} s_{*}\left(x^{\alpha}\right) \leq \frac{3}{2}\right.\right.
$$

for $\left(\tau, x^{\alpha}\right) \in \operatorname{supp} q_{4 \epsilon}$.

(iii) We choose on $V^{\prime} \times[0, \infty[$ a collection $v$ of smooth fields analogous to those represented by $u$ which satisfy the boundary condition (7.1) on $I^{\prime}=\mathcal{I} \cap\left(V^{\prime} \times[0, \infty[)\right.$, which coincide with the data $u_{0}$ on $V^{\prime}$, and for which the respective components take on $\mathcal{I}^{\prime} \cap$ supp $q_{4 \epsilon}$ the values (5.46), (5.47).

(iv) In the part of the boundary adapted reduced field equations given by (5.33) we replace the term $A^{3}=A^{a b} c_{a b}^{3}$ by $A^{a b}\left(q_{\varepsilon} c^{3} a b+\left(1-q_{\varepsilon}\right) e_{a b}\right)$ with $e_{a b}=-\rho_{a b} / \sqrt{2}=$ $\left.c^{3}{ }_{a b}\right|_{\partial S}=$ const.

(v) After this we replace in all equations $u, \Omega$, and $d_{a b}$ respectively by $u+q_{2 \varepsilon} v, q_{2 \varepsilon} \Omega$, $q_{2 \varepsilon} d_{a b}$ and denote the functions $q_{\varepsilon}, q_{2 \varepsilon} v, q_{2 \varepsilon} \Omega, q_{2 \varepsilon} d_{a b}$ in the equation collectively by $a$. We extend these functions by value zero to $\left\{x \in \mathbb{R}^{4} \mid x^{0}, x^{3} \geq 0\right\}$ to obtain smooth functions of compact support in $\left\{x \in \mathbb{R}^{4} \mid x^{0}, x^{3} \geq 0\right\}$.

(vi) Observing the reality conditions which are to be satisfied by the various components of the fields comprised by $u$, we finally write the resulting equations as a real symmetric hyperbolic system for an $\mathbb{R}^{N}$-valued unknown which we denote again by $u$. The $\mathbb{R}^{N^{\prime}}$. valued function appearing in the equations will again be denoted by $a$.

We have reformulated the problem in such a way that there exists a neighbourhood of the point $x^{\mu}=0$ in $\left\{x \in \mathbb{R}^{4} \mid x^{0}, x^{3} \leq 0\right\}$ on which the original problem is equivalent to the initial boundary value problem defined by (8.2)-(8.4).

The reformulation shows that our problem is close to a class of problems discussed by Guès [9]. In fact, we have achieved almost the same form of the problem as considered in that article. There is a slight but important difference. In the article by Guès it is assumed that the basic properties of the normal matrix $A^{3}$ are determined on $\left\{x^{3}=0, x^{0} \geq 0\right\}$ by the boundary conditions. In the present case the normal matrix is determined by Eq. (8.2). However, we have perfect control on it. By Lemma 5.1 the normal matrix can be determined by integrating the system of ordinary differential equations (5.43)-(5.45). After our reformulation we find that the normal matrix in Eq. (8.2) is essentially given by 


$$
A^{3}(a, u)=f\left(x^{\mu}\right) A_{0}^{3} \quad \text { on }\left\{x^{3}=0, x^{0} \geq 0\right\}, \quad f=1-q_{\epsilon} \frac{\frac{1}{2} \tau^{2} s_{*}}{1+\frac{1}{2} \tau^{2} s_{*}},
$$

where we denote by $A_{0}^{3}$ the value of the matrix (5.42) at $\tau=0$. It follows that $f$ is a smooth function satisfying $\frac{2}{3} \leq f \leq 2$ on $\left\{x^{3}=0, x^{0} \geq 0\right\}$. If one studies, following the procedure in [9], in an iteration procedure the sequence of linear problems

$$
A^{\mu}\left(a, u_{k}\right) \partial_{\mu} u_{k+1}=F\left(a, \partial_{\nu} a, u_{k}\right), \quad k=1,2,3, \ldots
$$

for an unknown $u_{k+1}$, where the iteration is started with the function $u_{1}$ which vanishes everywhere, it follows for any $k$, that the normal matrix of Eq. (8.6) is given by (8.5). Consequently every step in Guès' discussion can be applied to the present situation. Since for the situation considered here nothing can be gained in the smooth case which would go beyond the results obtained by Guès, we refrain from repeating the details. Theorems 3 and 4 in [9] imply the following theorem.

Theorem 8.2. There exists a number $\alpha>0$ for which there exists a unique smooth solution $u$ of the problem (8.2)-(8.4).

We could deduce from [9] a result involving weaker differentiability assumptions. It should be noted that the smoothness results near the boundary obtained for the general case studied in [9] may then be slightly improved for our particular problem.

Finally, we want to point out that the extensibility Theorem 4 in [9] is only of restricted applicability in our situation. On the one hand we know from the discussion in Section 4.1 that even if the solution considered above would exist for arbitrary values of the coordinate $\tau$, the solution might still be extensible after a coordinate transformation. On the other hand it may happen that some of the fields considered in our representation of the field equations blow up after a finite "time" $\tau$ because the conformal geodesics form caustics, while the underlying space-time might still be extensible as a solution to the conformal field equations.

\section{References}

[1] L. Andersson and P.T. Chruściel, On the asymptotic behaviour of solutions to the constraint equations in general relativity with "hyperboloidal" boundary conditions, preprint (1994).

[2] L. Andersson, P.T. Chruściel and H. Friedrich, On the regularity of solutions to the Yamabe equation and the existence of smooth hyperboloidal initial data for Einstein's field equations, Commun. Math. Phys. 149 (1992) 587-612.

[3] E. Cartan, Les espaces à connexion conforme, Ann. Soc. Po. Math. 2 (1923) 171-221.

[4] Y. Choquet-Bruhat and J.W. York, Jr., The Cauchy problem, in: General Relativity and Gravitation, Vol. I, ed. A. Held (Plenum Press, New York, 1980).

[5] H. Friedrich, On the existence of $n$-geodesically complete or future complete solutions of Einstein's field equations with smooth asymptotic structure, Commun. Math. Phys. 107 (1986) 587-609.

[6] H. Friedrich, On static and radiative spacetimes, Commun. Math. Phys. 119 (1988) 51-73.

[7] H. Friedrich, On the global existence and the asymptotic behaviour of solutions to the EinsteinMaxwell-Yang-Mills equations, J. Diff. Geom. 34 (1991) 275-345. 
[8] H. Friedrich and B.G. Schmidt, Conformal geodesics in general relativity, Proc. Roy. Soc. Lond. A 414 (1987) 171-195.

[9] O. Guès, Problème mixte hyperbolique quasi-linéaire charactéristique, Commun. Part. Diff. Equ. 15 (1990) 595-645.

[10] S.W. Hawking, The boundary conditions for gauged supergravity, Phys. Lett. 126 B (1983) 175-177.

[11] S.W. Hawking and F.G.R. Ellis, The Large Scale Structure of Space-Time (Cambridge University Press, Cambridge, 1973).

[12] M. Henneaux and C. Teitelboim, Asymptotically Anti-de Sitter spaces, Commun. Math. Phys. 98 (1985) $391-424$.

[13] S. Kobayashi, Transformation Groups in Differential Geometry (Springer, Berlin, 1972).

[14] K. Ogiue, Theory of conformal connections, Kodai Math. Sem. Rep. 19 (1967) 193-224.

[15] R. Penrose, Conformal treatment of infinity, in: Relativity, Groups and Topology, Les Houches 1963, eds. B. de Witt and C.M. de Witt (Gordon and Breach, New York, 1964).

[16] R. Penrose, Zero rest-mass fields including gravitation: asymptotic behaviour, Proc. Roy. Soc. Lond. A 284 (1965) 159-203.

[17] R. Penrose, Structure of space-time, in: Battelle Rencontres, 1967 Lectures in Mathematics and Physics, eds. C.M. de Witt and J.A. Wheeler (Benjamin, New York, 1968).

[18] R. Penrose and W. Rindler, Spinors and space-time, Vols. I, II (Cambridge University Press, Cambridge, 1986).

[19] J. Rauch, Symmetric positive systems with boundary characteristics of constant multiplicity, Trans. Amer. Math. Soc. 291 (1985) 167-187.

[20] M.E. Taylor, Pseudodifferential Operators and Nonlinear PDE (Birkhäuser, Boston, 1991).

[21] H. Weyl, Reine Infinitesimalgeometrie, Mathem. Zeitschrift 2 (1918) 384-411.

[22] K. Yano, Sur les circonférences généralisées dans les espaces à connexion conforme, Proc. Imp. Acad. Tokyo 14 (1938) 329-332; Sur la théorie des espaces à connexion conforme, J. Fac. Sci. Univ. Tokyo Sect. 1, 4 (1939) 1-59. 